University of Tennessee Health Science Center

UTHSC Digital Commons

\title{
Using Grounded Theory to Reveal Tacit Knowledge for Nurse Educators in an Unfamiliar African Setting
}

\author{
Ramona B. Patterson \\ University of Tennessee Health Science Center
}

Follow this and additional works at: https://dc.uthsc.edu/dissertations

Part of the Nursing Commons

\section{Recommended Citation}

Patterson, Ramona B. , "Using Grounded Theory to Reveal Tacit Knowledge for Nurse Educators in an Unfamiliar African Setting" (2012). Theses and Dissertations (ETD). Paper 205. http://dx.doi.org/ 10.21007/etd.cghs.2012.0239.

This Dissertation is brought to you for free and open access by the College of Graduate Health Sciences at UTHSC Digital Commons. It has been accepted for inclusion in Theses and Dissertations (ETD) by an authorized administrator of UTHSC Digital Commons. For more information, please contact jwelch30@uthsc.edu. 


\title{
Using Grounded Theory to Reveal Tacit Knowledge for Nurse Educators in an Unfamiliar African Setting
}

\begin{abstract}
There is a gap in evidence to guide individuals as they prepare for a nursing education partnership in an unfamiliar setting. Much of the existing knowledge base is contained within institutional or organizational confines. Individuals new to the field need access to existing expertise and tacit knowledge. The purpose of this research was to generate a grounded theory to guide aspiring nursing faculty in developing the necessary knowledge base to fulfill the nurse educator role in an unfamiliar setting. Ghana, Africa, was specified as the unfamiliar setting and context for this study.

Purposive sampling identified individuals from North America and Ghana who were experienced in the role preparation processes of outside nurse educators partnering in Ghana. In addition to first-hand experience with the phenomenon of interest, inclusion criteria specified the ability to speak and converse in English, and a minimum age of 18. Data collection used theoretical sampling via telephone interviews for individuals from North America $(n=10)$ experienced as partners for nursing education in Ghana. After determining theoretical saturation from North American participants, sampling transitioned via researcher fieldwork, for face-toface interviews with individuals from inside Ghana $(n=6)$ experienced in partnering with outsiders for nursing education. Data management included an inductive on-going data analysis process that began with the first interview, and used constant comparison of thematic concepts until the final theory emerged. Interviews were audio-recorded, and transcribed into Microsoft Word. Member checks served to validate the transcriptions. Researcher thoughts, observations, and methodological decisions were recorded in journal entries in memo format, and served as another data source for this study. TAMS Analyzer qualitative software was used to store, manage, code, and analyze the data. An audit trail was used to track data sources.
\end{abstract}

Findings revealed five steps to outsider preparation processes that organized the emergent theory, Preparing for an Unfamiliar Setting, grounded in participant experiences. The five steps included (1) Personalizing Information Needs, (2) Seeking Information, (3) Laying the Groundwork, (4) Developing Understanding, and (5) Framing the Outsider Role. Antecedents to preparation processes included the participants' past experience, existing network, and existing knowledge base. Progression through the process was fluid, allowing individualized interpretation based on antecedent characteristics. Instances of ineffective preparation were also revealed. Findings indicated that outsiders returned to earlier steps in the process when they recognized a gap in knowledge or understanding. Failure to correct gaps in understanding or amend gaps in knowledge resulted in ineffective partnering while Framing the Outsider Role. Findings revealed the necessity of including insiders as information resources during preparation.

This research generated a theory grounded in the realities of individuals' experiences while partnering for nursing education in an unfamiliar setting. Concepts derived from interview data combined with researcher interpretations formed a theoretical framework that can guide individual nurses through the process of Preparing for an Unfamiliar Setting

\section{Document Type}

Dissertation

Degree Name

Doctor of Philosophy (PhD)

Program

Nursing 
Research Advisor

Michael A. Carter, DNSc, DNP

\section{Keywords}

grounded theory, nurse educator, qualitative research, role translation, tacit knowledge, unfamiliar setting

\section{Subject Categories}

Medicine and Health Sciences | Nursing 


\title{
USING GROUNDED THEORY TO REVEAL TACIT KNOWLEDGE FOR NURSE EDUCATORS IN AN UNFAMILIAR AFRICAN SETTING
}

\author{
A Dissertation \\ Presented for \\ The Graduate Studies Council \\ The University of Tennessee \\ Health Science Center
}

\author{
In Partial Fulfillment \\ Of the Requirements for the Degree \\ Doctor of Philosophy \\ From The University of Tennessee
}

By

Ramona B. Patterson

December 2012 
Copyright (C) 2012 by Ramona B. Patterson. All rights reserved. 


\section{DEDICATION}

This dissertation is dedicated to my family, both physical and spiritual, to God the Father, Christ the Son, and the Holy Spirit the Comforter, without whom this work would not have been completed. To God be the glory. 


\section{ACKNOWLEDGEMENTS}

There have been many people who have helped me along my dissertation journey. I wish to express my sincere appreciation and heartfelt gratitude to all who have made the journey with me. I want to begin by thanking the people who have helped guide me through the dissertation process. Dr. Michael Carter, thank you for assuming the role of advisor and chair, your supportive guidance helped focus and reframe my research. Thank you to my UTHSC committee members, Dr. Susan Jacob, Dr. Margaret Hartig, and Dr. Patricia Speck for the expertise and guidance you provided. A special thanks to my external committee member Dr. Lynda Wilson, deputy director of the World Health Organization Collaborating Center on International Nursing at the University of Alabama at Birmingham, for your expertise, insights, and guidance. I also want to thank Dr. Cynthia Russell for getting me started on this journey, and helping me identify a research interest that could become a life's work.

I would also like to express my gratitude to individuals who were instrumental to the research effort. I want to thank Dr. Augustine Tawiah for prompting my initial interest in Ghana, facilitating my efforts there, and serving as my host and in-country liaison while I was collecting data in Ghana. I thank the Ghanaian people and governmental officials who supported my efforts and provided access to key informants in Ghana for my study. I also thank the nurse educators in North America and Ghana who freely gave of their time to contribute to this research.

On a personal note, I thank my husband Lynn, for supporting my efforts to undertake this work, financing the first trip to Ghana, and for encouraging me to complete the research. I thank my children and their families for helping me seek balance throughout the journey. I also thank my parents, relatives, and friends for listening, praying, and simply being there when the journey got rough. 


\begin{abstract}
There is a gap in evidence to guide individuals as they prepare for a nursing education partnership in an unfamiliar setting. Much of the existing knowledge base is contained within institutional or organizational confines. Individuals new to the field need access to existing expertise and tacit knowledge. The purpose of this research was to generate a grounded theory to guide aspiring nursing faculty in developing the necessary knowledge base to fulfill the nurse educator role in an unfamiliar setting. Ghana, Africa, was specified as the unfamiliar setting and context for this study.
\end{abstract}

Purposive sampling identified individuals from North America and Ghana who were experienced in the role preparation processes of outside nurse educators partnering in Ghana. In addition to first-hand experience with the phenomenon of interest, inclusion criteria specified the ability to speak and converse in English, and a minimum age of 18. Data collection used theoretical sampling via telephone interviews for individuals from North America $(\mathrm{n}=10)$ experienced as partners for nursing education in Ghana. After determining theoretical saturation from North American participants, sampling transitioned via researcher fieldwork, for face-to-face interviews with individuals from inside Ghana $(\mathrm{n}=6)$ experienced in partnering with outsiders for nursing education. Data management included an inductive on-going data analysis process that began with the first interview, and used constant comparison of thematic concepts until the final theory emerged. Interviews were audio-recorded, and transcribed into Microsoft Word. Member checks served to validate the transcriptions. Researcher thoughts, observations, and methodological decisions were recorded in journal entries in memo format, and served as another data source for this study. TAMS Analyzer qualitative software was used to store, manage, code, and analyze the data. An audit trail was used to track data sources.

Findings revealed five steps to outsider preparation processes that organized the emergent theory, Preparing for an Unfamiliar Setting, grounded in participant experiences. The five steps included (1) Personalizing Information Needs, (2) Seeking Information, (3) Laying the Groundwork, (4) Developing Understanding, and (5) Framing the Outsider Role. Antecedents to preparation processes included the participants' past experience, existing network, and existing knowledge base. Progression through the process was fluid, allowing individualized interpretation based on antecedent characteristics. Instances of ineffective preparation were also revealed. Findings indicated that outsiders returned to earlier steps in the process when they recognized a gap in knowledge or understanding. Failure to correct gaps in understanding or amend gaps in knowledge resulted in ineffective partnering while Framing the Outsider Role. Findings revealed the necessity of including insiders as information resources during preparation.

This research generated a theory grounded in the realities of individuals' experiences while partnering for nursing education in an unfamiliar setting. Concepts derived from interview data combined with researcher interpretations formed a theoretical framework that can guide individual nurses through the process of Preparing for an Unfamiliar Setting. 


\section{TABLE OF CONTENTS}

CHAPTER 1. INTRODUCTION ............................................................................ 1

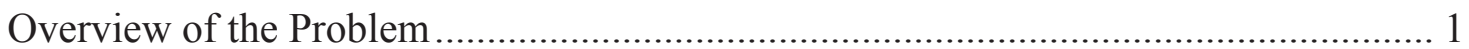

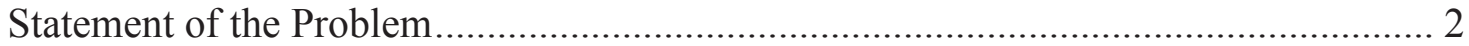

Purpose, Specific Aims, and Central Research Question .............................................. 2

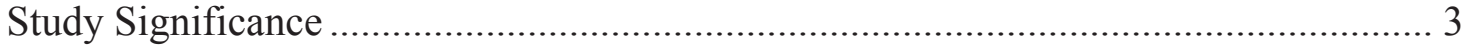

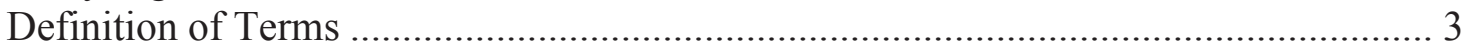

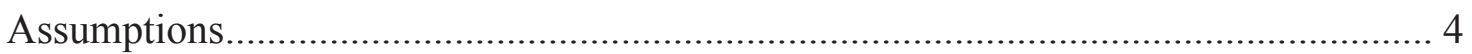

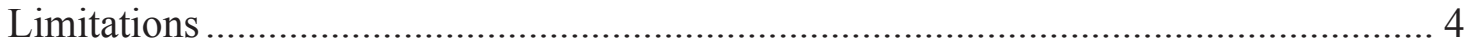

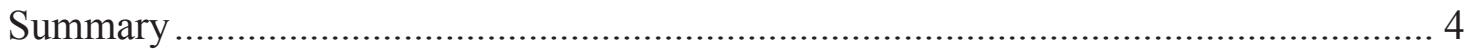

CHAPTER 2. BACKGROUND AND RESEARCH CONTEXT ESTABLISHED.... 6

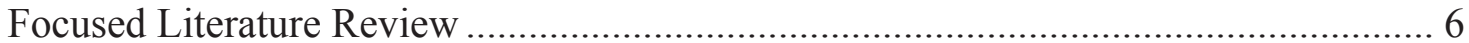

Faculty Preparation for Teaching in Unfamiliar Settings .................................... 6

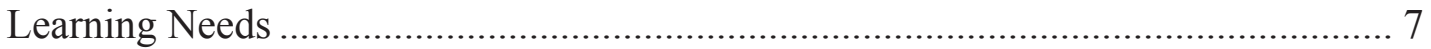

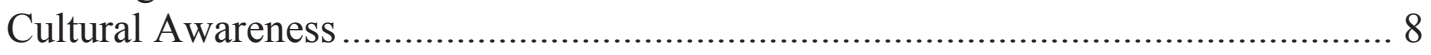

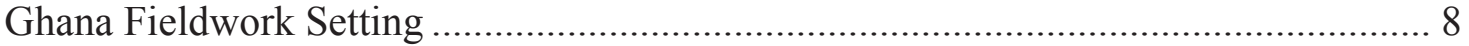

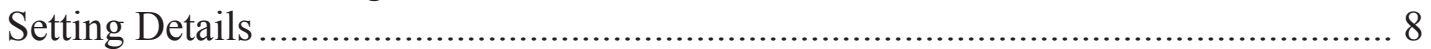

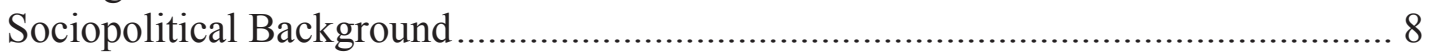

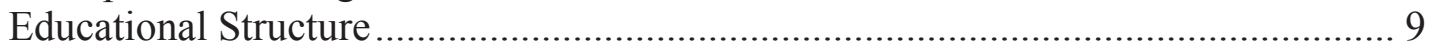

Setting for Nursing Shortage and Nurse Educator Shortage.................................. 9

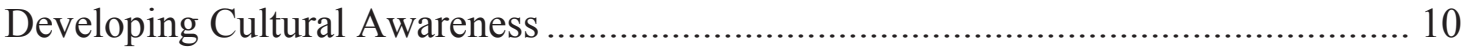

Integrated Review of Health Disparities in Ghana ............................................. 10

Developing Awareness of Ghanaian Culture..................................................... 12

Preliminary Ethnographic Research ............................................................... 12

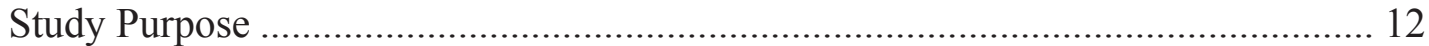

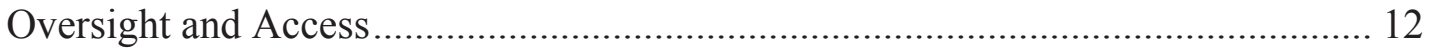

Data Sources and Analysis........................................................................... 14

Ethnographic Findings Organized ................................................................... 14

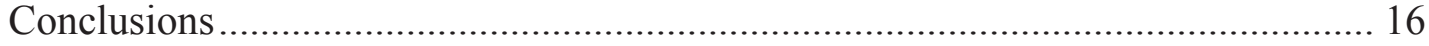

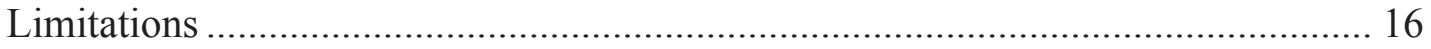

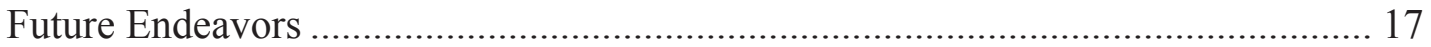

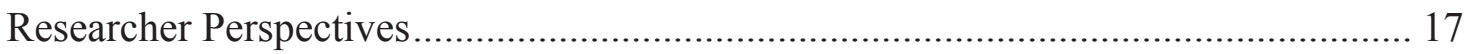

Personal Experiences and Perspectives............................................................ 17

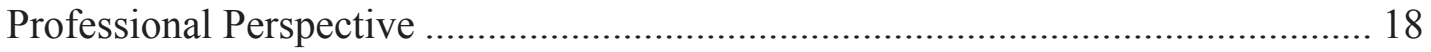

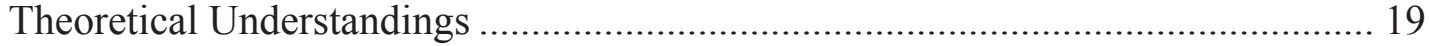

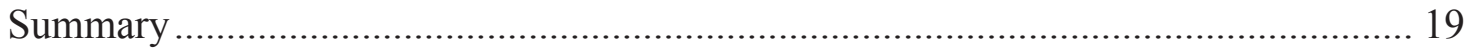

CHAPTER 3. METHODOLOGY ................................................................................. 20

Grounded Theory Overview .............................................................................. 20

Identification of the Unfamiliar Setting .............................................................. 20

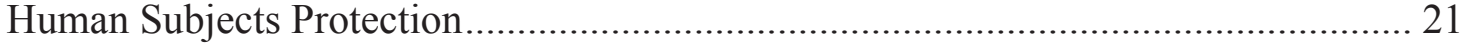

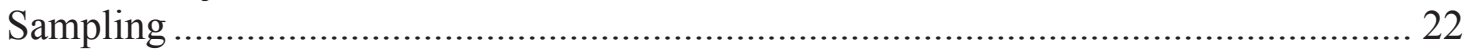

Identification of Target Sample ......................................................................... 23 
Participant Recruitment Procedures..................................................................... 24

Access and Support System in Ghana................................................................. 25

Data Collection Tools and Instrumentation ........................................................... 26

Methodological Rigor and Trustworthiness …………................................................ 27

Credibility .................................................................................................... 27

Transferability, Dependability, and Confirmability............................................... 28

Methodological Rigor for Study Limitations....................................................... 28

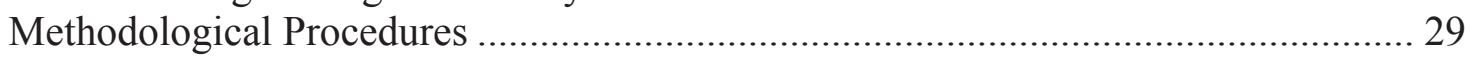

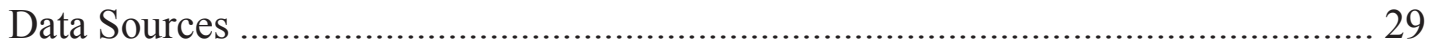

Data Collection for Interview Data ....................................................................... 30

Procedures for Storing and Transcribing Interview Data .......................................... 31

Data Collection and Storage for Researcher Generated Data ................................... 33

Data Management and Analysis ......................................................................... 33

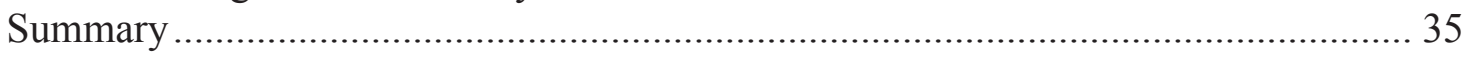

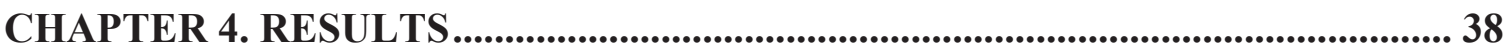

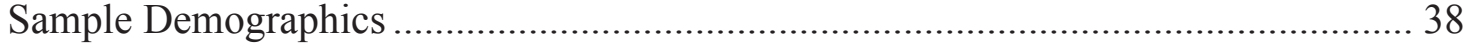

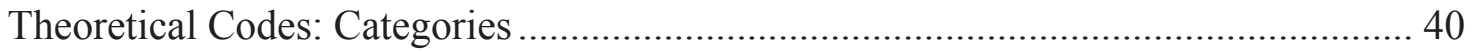

Category 1: Personalizing Information Needs....................................................... 40

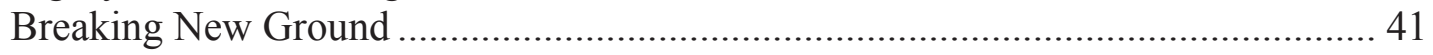

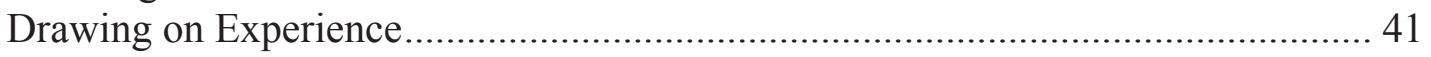

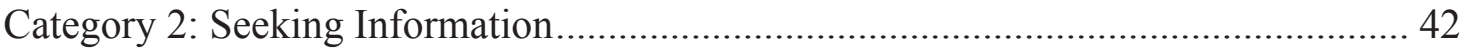

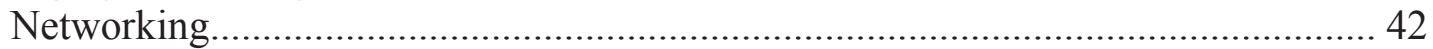

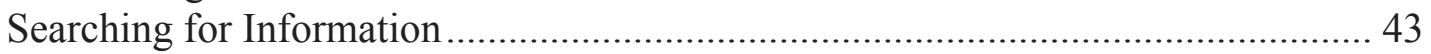

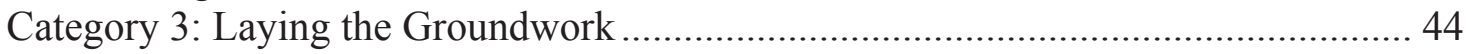

Identifying Information or Knowledge Needs ........................................................ 44

Identifying Key Information Resources............................................................. 48

Building Relationships ...................................................................................... 52

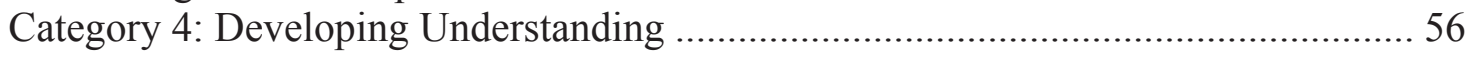

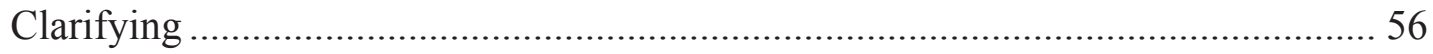

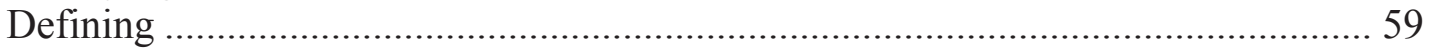

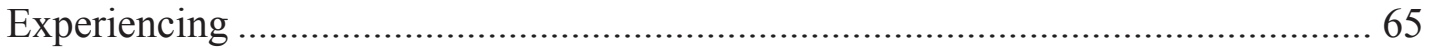

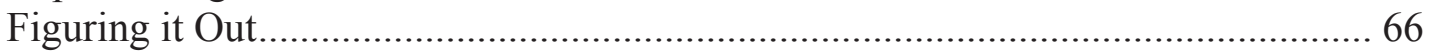

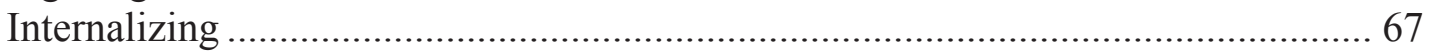

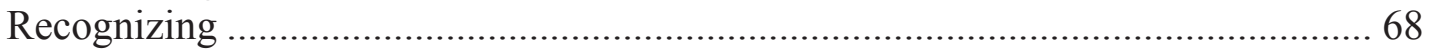

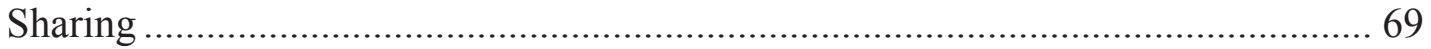

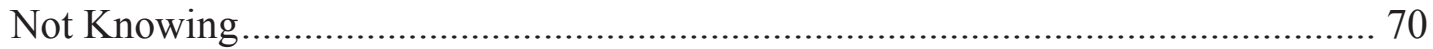

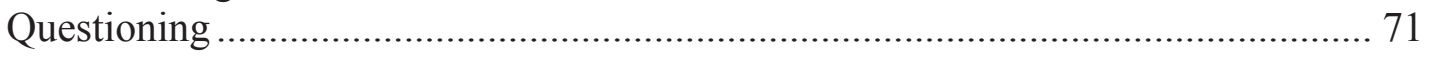

Category 5: Framing the Outsider Role ................................................................ 73

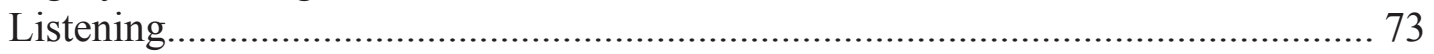

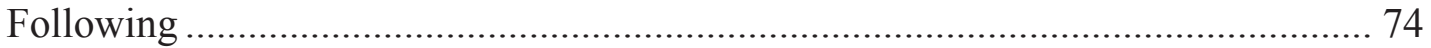

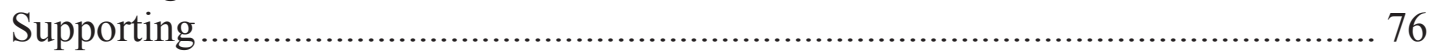

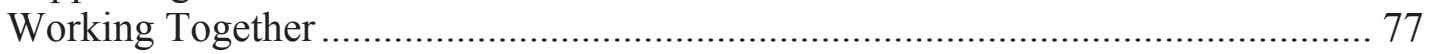

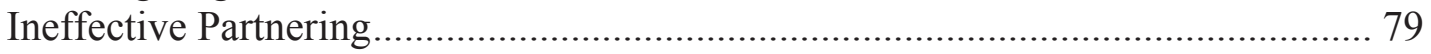

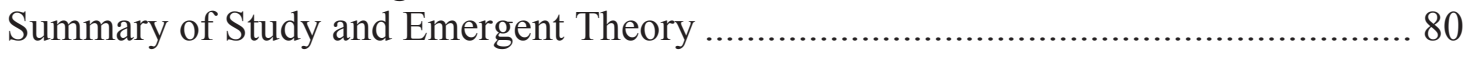

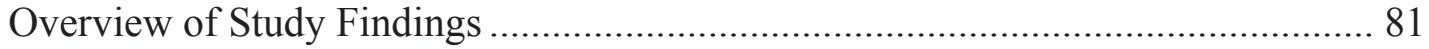


Theoretical Model

CHAPTER 5. DISCUSSION............................................................................................. 86

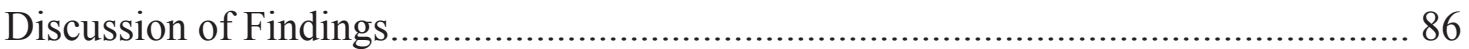

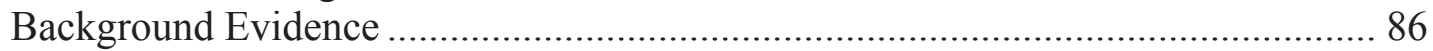

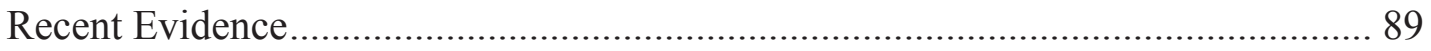

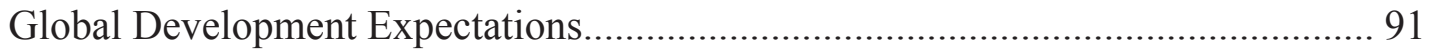

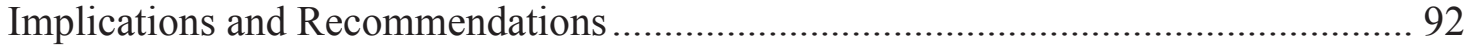

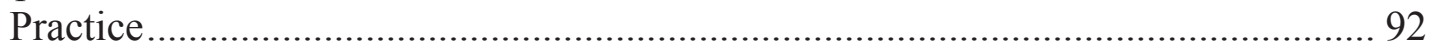

Theory Development and Future Research.......................................................... 97

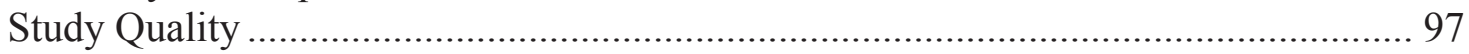

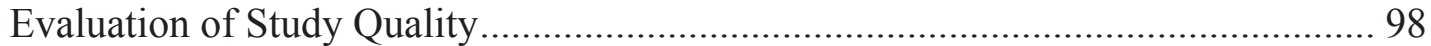

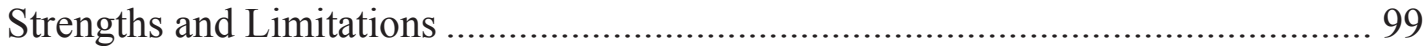

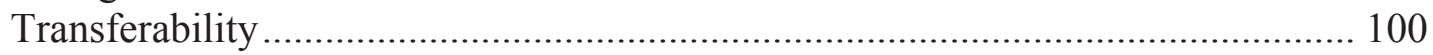

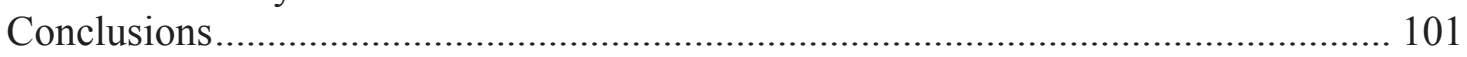

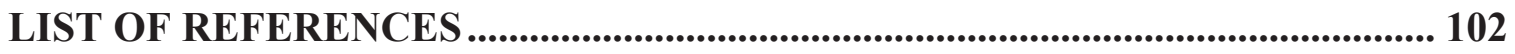

APPENDIX A. UTHSC IRB APPROVAL LETTER............................................... 115

APPENDIX B. CONSENT STATEMENT ....................................................... 117

APPENDIX C. LETTER OF INTENT TO NMCG ............................................. 118

APPENDIX D. LETTER OF INTENT TO GHANA MOH .................................. 119

APPENDIX E. LETTER OF INTENT TO GHANA MOE...................................... 120

APPENDIX F. MOH LETTER OF INTRODUCTION AND SUPPORT FOR

RESEARCH IN GHANA ................................................................................... 121

APPENDIX G. INITIAL EMAIL CONTACT SCRIPT ..................................... 122

APPENDIX H. FOLLOW-UP CONTACT SCRIPT ............................................ 123

APPENDIX I. BROAD INTERVIEW GUIDE .................................................. 124

APPENDIX J. TRANSCRIPTIONIST CONFIDENTIALITY STATEMENT..... 125

VITA 


\section{LIST OF TABLES}

Table 4-1. Sample demographics, Ghanaian and US participants $(\mathrm{n}=16) \ldots \ldots \ldots \ldots \ldots \ldots . . . . . . . .39$

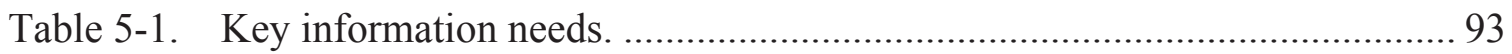

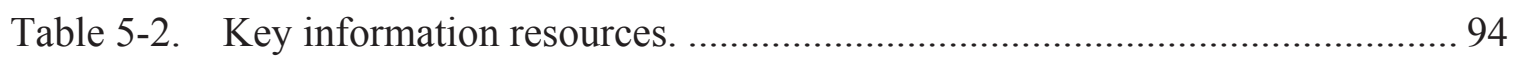

Table 5-3. Lessons learned: Barriers and facilitators to preparation process............... 95 


\section{LIST OF FIGURES}

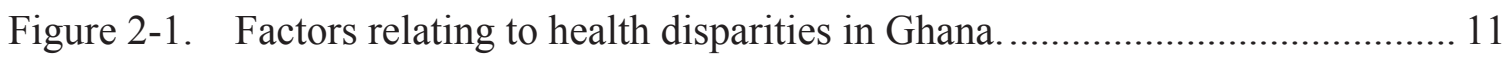

Figure 2-2. Societal factors for consideration when developing cultural awareness..... 13

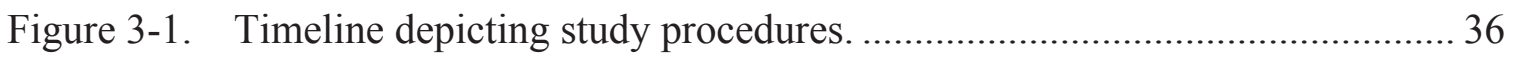

Figure 4-1. Substantive theory: Preparing for an Unfamiliar Setting ......................... 84 


\section{LIST OF ABBREVIATIONS}

$\begin{array}{ll}\text { CDC } & \text { Centers for Disease Control and Prevention } \\ \text { CIA } & \text { Central Intelligence Agency } \\ \text { GHWA } & \text { Global Health Workforce Alliance } \\ \text { HLF } & \text { High Level Forum on Aid Effectiveness } \\ \text { HRH } & \text { Human Resources for Health } \\ \text { ICN } & \text { International Council of Nurses } \\ \text { IRB } & \text { Institutional Review Board } \\ \text { MDGS } & \text { Millennium Development Goals } \\ \text { MOE } & \text { Ministry of Education } \\ \text { MOH } & \text { Ministry of Health } \\ \text { NGO } & \text { Non-Governmental Organization } \\ \text { NMCG } & \text { Nursing and Midwifery Council of Ghana } \\ \text { RN } & \text { Registered Nurse } \\ \text { UN } & \text { United Nations } \\ \text { US } & \text { United States } \\ \text { UTHSC } & \text { The University of Tennessee Health Science Center } \\ \text { WBG } & \text { World Bank Group } \\ \text { WHO } & \text { World Health Organization }\end{array}$




\section{CHAPTER 1. INTRODUCTION}

\section{Overview of the Problem}

There is a global shortage of qualified healthcare workers, serving as a major barrier for achievement of the United Nations (UN) Millennium Development Goals (MDGS) (Buchan \& Calman, 2005). Nurses comprise the largest portion of global healthcare workers, with a nursing shortage that is complicated by a corresponding shortage of qualified nurse educators (International Council of Nurses [ICN] \& Florence Nightingale International Foundation, 2006). Compounding the shortages, inequities in the distribution of healthcare workers and educators are magnified in low-resource countries when compared to countries with higher economic resources (Anyangwe \& Mtonga, 2007; Chen et al., 2004; World Health Organization [WHO], 2006).

In response to the shortages, the Global Health Workforce Alliance (GHWA, 2008b) proposed six strategies as a preliminary guide to address the global healthcare worker crisis. One of those strategies is focused on the need to scale up the provision of training and education for healthcare workers, via efforts that are congruent with nationally identified plans for a setting's prioritized healthcare needs. Acknowledging the need for global accountability, the Alliance (GHWA, 2008b) called for global response via international partnerships to address the healthcare worker crisis. Partnerships to address that crisis can involve institutions, organizations, and individual members of the global community. A growing body of evidence exists to link and guide interested institutions and organizations through the process of developing an international twinning partnership (Aiken \& Poghosyan, 2009; American International Health Alliance, 2006; Kohi, Portillo, Safe, Okonsky, Nilsson, \& Holzemer, 2010; Masera, et al., 1998; St Jude Children's Research Hospital, n.d.).

Individual members of the global community with specific skill sets and expertise want to become involved in partnering to support healthcare in different parts of the world. Existing resources primarily target institutional or organizational partnerships, with little published research to help individuals understand how they can equip themselves for a partnership in an unfamiliar setting. Nurse educators wishing to help with nursing education needs in another setting must understand the contexts for nursing education in that setting. Variations in contexts for nursing education, practice, academic preparation, and the standards for education and practice prohibit the rote transfer of nursing curriculum from one setting to another (Mooneyhan \& Campos, 1984). Individuals planning to work in an unfamiliar setting need help identifying essential knowledge to enable job performance (Murphy, 2008). A limited scientific knowledge base exists regarding the details of university cultures in low-resource countries, making it difficult for potential partners to understand and interpret the educational needs in context with the realities of the setting (King, 2009).

Partnerships for nursing education are one mechanism to address the human resources for health crisis, but the realities of nursing roles and scopes of practice in other 
countries are seldom defined clearly for understanding by those outside the community or country (Kuehn et al., 2005). Shifts in developmental aid for higher education prioritize collaborative efforts addressing crisis level needs in sub-Saharan African universities (King, 2009). Research is needed to enable understanding of the cultures and contexts in which potential educational partnerships would be expected to function (King, 2009).

\section{Statement of the Problem}

A problem exists regarding the limited amount of information available to guide individuals as they prepare for a nursing education partnership in an unfamiliar setting. Much of the existing knowledge base from past participants is contained within institutional or organizational confines as project reports, with little information published or disseminated to guide others through similar endeavors (Immonen, Andersson, \& Lvova, 2008; Salvage, 2007). Contained within those confines, the knowledge base is essentially hidden inside an invisible college. Zuccala (2006) specified that an invisible college is a group of scholars or scientists with formal and informal channels for communication, collaboration, and the transfer of knowledge relating to common research interests. Access to that college's knowledge and information is often limited (Cooper, 1998; Zuccala, 2006). Individuals new to the field need to be able to access the expertise and tacit knowledge from that invisible college. Existing publications provide insight to various partnership processes and select recommendations, but no publications were identified that explored the nursing profession's experiential and tacit knowledge concerning faculty preparation processes to facilitate the nurse educator role in unfamiliar settings. Research is needed in the field of nursing education, to develop an evidence-base of the professional community's tacit knowledge as a foundation to nursing education practices (Ferguson \& Day, 2005; Meerabeau, 1992).

\section{Purpose, Specific Aims, and Central Research Question}

The purpose of this research was to generate a grounded theory to guide aspiring nursing faculty in developing the necessary knowledge base to fulfill the nurse educator role in an unfamiliar setting. For this research, the unfamiliar setting has been specified as Ghana, Africa. Specific aims of this research were to:

1. Explore insider perspectives and tacit knowledge concerning the learning processes whereby nurse educators acquire the knowledge base to fulfill their roles in an unfamiliar setting.

2. Develop a grounded theory of how an outsider can identify relational tacit knowledge and make it explicit concerning faculty preparation processes for the nurse educator role in an unfamiliar setting. 
One central research question served to guide the study. That question was: By what processes do outside nurse educators learn how to fulfill the nurse educator role in an unfamiliar setting?

\section{Study Significance}

This study is significant because it adds to the evidence concerning tacit knowledge in nursing (Ferguson \& Day, 2005; Palmer \& Kramlich, 2011). It builds the science of nursing through theory development, grounded in experiential knowledge that can serve as a framework for future practice (Meerabeau, 1992; Whitehead, 2005).

Findings from this study add to the research base that guides nursing education practice (Diekelmann, Schulte, \& Ironside, 2002; Ferguson \& Day, 2005), particularly those educational practices in unfamiliar settings. The research is significant because it has been aligned with action recommendations from the Arista 3 conferences (DickensonHazard, 2004) to disseminate practice findings, promote communities of practice and international partnerships, and design nursing education to meet the needs and realities of the setting. The study is also significant because it has targeted research and partnerships in response to the global nurse educator shortage (GHWA, 2008b; Kulwicki, 2006). Finally, this research is significant beyond the field of nursing education. Through grounded theory research, it has addressed the gap in evidence (King, 2009) regarding the cultural contexts for higher education in low-resource countries and sub-Saharan Africa in particular.

\section{Definition of Terms}

The following terms have been defined to facilitate an understanding of key concepts in this study.

- Unfamiliar setting conveys the idea of an individual as a stranger in an environment for which societal norms and behaviors are unknown (Toumishey, 1989).

- Tacit knowledge refers to knowledge that is not explained, interpreted, or made explicit (Collins, 2010).

- Relational tacit knowledge is a form of tacit knowledge that relies on societal organization, and presumes the existence of sufficient cultural similarities among members of that society to facilitate understanding and transfer of knowledge between members (Collins, 2010). 


\begin{abstract}
Assumptions
Suppositions relevant to this research include assumptions related to basic educational principles, cultural influences, multiple realities, and experiential understandings. They have been based on heuristic understanding of the nurse educator role, preliminary literature review, principles of relational tacit knowledge (Collins, 2010) and principles of grounded theory research (Corbin \& Strauss, 2008). Assumptions relating to developing an understanding and interpreting the contexts for nursing education in an unfamiliar setting have included four elements.
\end{abstract}

1. Existing standards and curriculum guidelines for nursing education in that setting will need to be considered.

2. Cultural realities for nursing education and practice will include national interpretations that will influence the way nursing education is delivered.

3. Common understandings will exist across the group (society) of partners for nursing education.

4. Individual participants will have unique understandings relative to their own situational experiences.

\title{
Limitations
}

This research has several limitations. I am a novice to the grounded theory research design, with limited qualitative research experience - providing potential for less trustworthiness. A related limitation was the potential for investigator interactions/ interpretations to bias the results. Other limitations included the potential for interviewee response bias related to variations in roles/ worldviews and the societal influences of individual participants, as well as language barriers. English is the official language in the targeted country and widely used for schools and businesses; but preliminary fieldwork experiences revealed the need for additional time during interview communications, to ensure accurate interpretation of meanings. Mechanisms used to address the limitations are presented under Methodology (see Chapter 3).

\section{Summary}

There is a global shortage of healthcare workers. Compounding that shortage, there is a shortage of qualified nurse educators that directly impacts a community's ability to educate enough nurses to help fill their community's healthcare workforce needs. The GHWA (2008b) called for international partnerships to address the healthcare worker crisis. Although models exist for twinning partnerships between organizations in low resource countries and their counterpart organizations from higher resource countries (World Bank Group [WBG], n.d.), limited information exists to guide individual nurse 
educators as they prepare for partnerships in unfamiliar settings. Research is needed to help guide individuals as they prepare. This research has generated a theory grounded in the realities of individuals' experiences while participating in nursing education partnerships in an unfamiliar setting. Concepts derived from interview data have been combined with literature evidence and researcher insights, to form a theoretical framework that can guide individual nurse educators through preparation processes that can help equip them for the nurse educator role in an unfamiliar setting. 


\section{CHAPTER 2. BACKGROUND AND RESEARCH CONTEXT ESTABLISHED}

This chapter begins with a focused literature review, in keeping with guidelines for grounded theory research (Corbin \& Strauss, 2008), as background evidence to support the research question, preliminary concepts, and sampling strategies. Next, background information relevant to the unfamiliar setting chosen for this study has been presented. Part of the setting background includes a report of preliminary research findings from an ethnographic study I conducted in 2008, adding first-hand evidence of the research context for this study. Last, researcher perspectives are presented, offering insight to personal worldviews, while fostering awareness that promotes sensitivity to the data.

\section{Focused Literature Review}

\section{Faculty Preparation for Teaching in Unfamiliar Settings}

Nurse educators in unfamiliar settings. Literature findings relevant to this study focused on information resources to guide nursing faculty members through information acquisition to fulfill the nurse educator role in an unfamiliar setting. Publications focused on guiding nurse educators through the process of developing the necessary knowledge base for role performance in an unfamiliar international setting (Desantis, 1995; Lange \& Ailinger, 2001) are limited, and pre-date the UN plan (Millennium Project, 2006) for achieving the MDGS. Desantis (1995) provided a model that details tasks for donor and host groups in a counterpart relationship where expert donors share their knowledge and expertise with host recipients in a way that fits the cultural setting. Donors were charged with learning about a) the social and cultural contexts of the host group, b) academic expertise and specialized knowledge of host group, c) the host educational system and methods for teaching and learning within that system, and d) previous counterpart activities with which the host group had been associated, and similar counterpart activities in other settings (Desantis, 1995). Lange and Ailinger (2001) presented a fivepart model for international nursing faculty exchanges: "pre-exchange planning, academic activities, socio-cultural events, communication, and evaluation of outcomes" (p. 110). They provided a checklist of information the visiting nurse educator needed from the host, stressed the need for culturally appropriate interactions, and recommended preparatory learning about the host country, institution, and faculty members (Lange \& Ailinger, 2001).

Although published after the UN plan was commissioned in 2002, interviews by Melby, Dodgson, and Tarrant (2008) took place while the Millennium Project (2006) was being piloted in select countries. Their phenomenological study reported the experiences of eight nurse educators from the Western world who had taught in East Asia for over two years (Melby, Dodgson, \& Tarrant, 2008). Their research helped illuminate tacit meanings of nurse educator experiences when teaching in an unfamiliar setting, and 
categorized participant difficulties understanding the nurse educator role and educational systems under the theme of bridging pedagogies (Melby, Dodgson, \& Tarrant, 2008). Beyond descriptions of individual or organizational international nursing education project experiences, their study provided the only research evidence that revealed the tacit knowledge of multiple nurse educators experienced with teaching in unfamiliar settings; however, it did not reveal the process by which they gained the knowledge they needed to fulfill their roles.

Related resources, other perspectives. Haussler (2006) presented information from the perspective of both sides in a faculty exchange, sharing experiences hosting nurse visitors from abroad, and her experiences in Finland as a Fulbright Scholar. Preplanning recommendations for visiting nurses included learning about a) the host institution and country, b) cultural norms, c) curriculum components, d) teaching resources, e) expectations for day-to-day living, and f) language or communication requirements (Haussler, 2006). From an allied health perspective, Shah, Robinson, and Enezi (2002) presented a global-centric model for international health education partnerships. They proposed a bilateral exchange of information that is mutually beneficial to partners and reflective of global citizenship and principles of social justice rather than colonialism (Shah, Robinson, Enezi, 2002). Their model promotes cultural sensitivity and recommends learning to acquire a background understanding about the other partner's a) historical, b) sociopolitical, c) philosophical, d) economic, e) cultural, and f) national contexts. They also highlight the need to respect and understand each partner's educational or skill level, technological capability, and worldview (Shah, Robinson, \& Enezi, 2002).

The counterpart relationship is representative of the traditional approach for developmental aid to low-resource countries, in which a one-way transfer of aid suggests a relationship of superiority and dependability (Wright, Zerbe, \& Korniewicz, 2001). Since the adoption of the MDGS, shifts in developmental aid have focused on capacity building and developing partnership relationships in support of MDGS attainment (King, 2009). The newer approach for developmental aid fosters an equitable relationship between partners, and considers the multidimensional contexts of sociopolitical, economic, cultural, and military influences on global and national health care needs (Wright, Zerbe, \& Korniewicz, 2001).

\section{Learning Needs}

The majority of publications concerning international nursing education partnerships describe program outcomes in relation to project aims (Girot \& Enders, 2004), with select insights into partner preparation. Tlou (1998) described developing an international nursing education partnership as a series of multiple processes rather than procedural tasks, and recommended that the process of initiating a partnership begin with learning about partners' institutional and human resources. Other authors indicated the need to learn about the contexts for health care and education (Byrne, 1998; Carty \& White, 1996; Edwards, Bunn, Mei, \& Hui, 1999; Johnson, Ghebreyohanes, Cunningham, 
Kutenplon, \& Bouey, 2007), existing resources (Byrne, 1998; Johnson et al., 2007), learner characteristics (Byrne, 1998; Johnson et al., 2007), curriculum needs (Bryne, 1998; Johnson et al., 2007), nursing roles and scopes of practice (Kuehn et al., 2005), and sociopolitical influences on the health care environment (Girot \& Enders, 2004).

\section{Cultural Awareness}

Many authors have validated the need for cultural awareness training and education before embarking upon a nursing education endeavor in an unfamiliar setting. Various topics were identified through literature reviews, as relevant to cultural awareness. Those topics included issues relating to sociopolitical and economic factors, safety considerations (traveler advisories, health promotion/ disease prevention, immunization needs, global health insurance, and medical evacuation arrangements), communication concerns, ethical considerations/variations in oversight, differing scopes of practice, different worldviews, and the need for local contacts to provide insights while networking and building trust between partners (Bosworth et al., 2006; Callister \& Cox, 2006; Crigger \& Holcomb, 2007; Edwards, Bunn, Morales-Mann, Papai, \& Davies, 2000; Harrison \& Malone, 2004; Kim, Woith, Otten, \& McElmurry, 2006; Lutzen, 2000; Perry \& Mander, 2005).

\section{Ghana Fieldwork Setting}

This section presents setting details, background information, and an overview of preliminary research in Ghana during 2008, helping illuminate contextual considerations for this study. Contextual considerations require reflective awareness to the interactions,

worldviews, and perspectives of the researcher and study participants throughout the data collection and analysis process for grounded theory research (Corbin \& Strauss, 2008).

\section{Setting Details}

The unfamiliar setting specified for this research is Ghana, West Africa. Geographically, Ghana's southern border is situated along the coastline of West Africa. Remaining boundaries include a western border with Togo, an eastern border with Cote d'Ivoire (Ivory Coast), and a northern border with Burkina Faso (Berry, 1994; Ghana Embassy, 2004-2010). With a location near the equator, Ghana has a tropical climate of dry and rainy seasons (Berry, 1994; Central Intelligence Agency [CIA], 2012).

\section{Sociopolitical Background}

In 1482, the area was occupied by Europeans and later fell to colonial rule in 1874 as the British Gold Coast Colony. The people gained independence from British rule on March 6, 1957 renaming their country Ghana (Berry, 1994; CIA, 2012; Ghana Embassy, 
2004-2010). In 1983 Ghana achieved political stability, with its current democracy demonstrating a growing economy and effective management of foreign aid as the nation endeavors to address inequities and growth constraints (WBG, 2010).

\section{Educational Structure}

Formalized education was initiated by colonial government during the $1600 \mathrm{~s}$, and was continued through the establishment of mission schools in Ghana (Eyiah, 2004). Ghana's Ministry of Education (MOE) implemented educational reform in 1987, transitioning from a British model for education to one conforming to the West African Examinations Council, and including a senior secondary school component (Keteku, 1999). Although challenged by resource inequities (rural - urban and north - south) following independence, educational development became a priority focus, with government-provided compulsory education available (Eyiah, 2004). Following the mandated basic public education (primary and junior secondary school), students take a basic education certification exam with scores determining which students advance to senior secondary school (Keteku, 1999). With each educational progression, students face increased competition for public education because of the limited number of educational facilities to service each progression (United States State Department Bureau of African Affairs [US SD BAA], 2010; US State Department [US SD], n. d.). Scores on senior secondary exams transmit to a hierarchy for post-secondary education options, since students who do not score high enough to get a university placement at one of the five public universities can make application to non-degree post- secondary educational programs (Keteku, 1999; US SD BAA, 2010; US SD, n. d.). Non-degree post-secondary programs at public institutions in Ghana include polytechnic institutions (10), teachers' training institutions (40), and nurses' training institutions (15) (US SD BAA, 2010; US SD, n. d.).

\section{Setting for Nursing Shortage and Nurse Educator Shortage}

Like many countries in sub-Saharan Africa, Ghana has been classified at a critical needs level for human resources for health $(\mathrm{HRH})$, lacking adequate numbers of HRH to serve societal health needs or meet MDGS for health (WHO, 2006). Ghana's most recent nurse - population ratio was recorded as 10.5 nurses/ midwives per 10,000 population (WHO, 2009). Core indicators related to MDGS illustrate costs to health in Ghana relevant to inadequate HRH. Latest available statistics for Ghana revealed that 74 children under 5 years of age die for every 1000 live births; $35 \%$ of the population with advanced human immunodeficiency virus receive coverage for antiretroviral therapy; and, 1,071,637 cases of malaria were reported during 2010 from a national population of 24,392,000. (WHO, 2010).

After recognizing the need to address health workforce concerns in 2005, Ghana acknowledged HRH as a top priority, began to develop a national health plan, and devised a strategic plan for HRH that was featured as a case study example for scaling up 
health worker training and education (GHWA, 2008a). Findings from the GHWA Ghana case study revealed strong political support for the national plan, inadequate numbers of qualified educators for all types of healthcare workers, the introduction of new types and titles of auxiliary healthcare workers to avoid confusion over what a nurse is, and a focus on pre-service educational interventions that targeted the realities of national needs for health care (GHWA, 2008a). Unfortunately, Ghana's initial plans for pre-service education to address their HRH crisis were halted by the lack of available qualified educators to serve their national needs (Anyangwe \& Mtonga, 2007). In partnership with the WHO, Quality Health Partners, and their affiliates, Ghana recently reviewed and revised assorted national curricula for various types of entry to practice and post-basic nursing education programs to better equip their nurses to meet the needs of their population (GNA, 2007, October 20).

\section{Developing Cultural Awareness}

\section{Integrated Review of Health Disparities in Ghana}

Purpose and search words. Reviewing current literature is one strategy for developing cultural awareness prior to visiting an unfamiliar setting (Callister \& Cox, 2006). During the fall of 2007, while preparing for preliminary research in Ghana, I conducted focused reviews to facilitate cultural understandings using Cooper's (1998) framework for integrative reviews. The purpose of the first review was to describe factors relating to health disparities in the country of Ghana, including issues relating to the nursing workforce and nursing education capabilities (Patterson \& Russell, 2008). Search words for that review included Ghana in combination with health problems, health disparities, health, health care needs, public health, nursing, nursing education, nursing workforce, and World Health Organization.

Literature findings. Data sources provided multidisciplinary evidence from the social sciences, medicine, and nursing. Literature findings were grouped and analyzed thematically (Patterson \& Russell, 2008). Analysis revealed four main themes: 1) major effects on health in Ghana, 2) factors contributing to health disparities in Ghana, 3) nursing workforce issues associated with health disparities in Ghana, and nursing education needs associated with health disparities in Ghana. Figure 2-1 shows the organizational structure of themes and sub-themes derived from the literature.

Gaps in evidence revealed. In addition to thematic findings, literature evidence revealed prior international nursing education partnerships at three of the four public universities in Ghana (Ogilvie, Allen, Laryea, \& Opare, 2003; Tabi \& Mukherjee, 2003; University of British Columbia, 2007). The fourth university was the target of my preliminary research in 2008. Findings from the review related to health disparities in Ghana revealed gaps in literature evidence to facilitate an outsider's understanding of nursing education needs as they are actualized for the realities of nursing practice in that setting. The gap in available evidence suggested a need to collaborate with Ghanaians to 


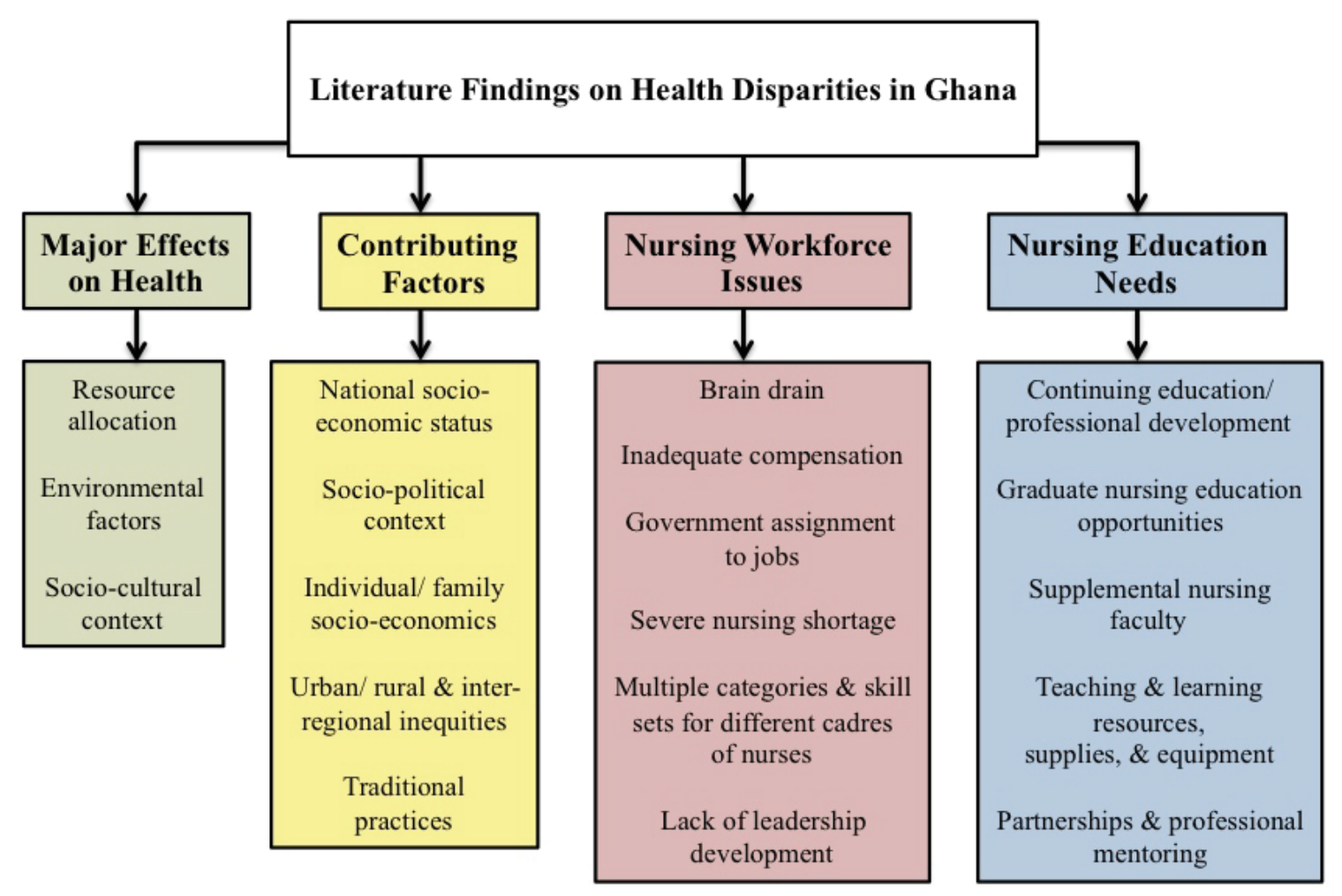

Figure 2-1. Factors relating to health disparities in Ghana.

Note: Factor categories and factor themes arose from literature findings in preparation for travel to Ghana in 2008.

Sources: Aiga, 2006; Aiga \& Kuroiwa, 2006; Akiwumi, 1994; Asante, Zwi, \& Ho, 2006; Centers for Disease Control and Prevention [CDC], 2007; Curtis, Garbrah-Aidoo, \& Scott, 2007; Dodoo, Adei, Couper, Hugman, \& Edwards, 2007; Dovlo, 2003; Dovlo, 2005; GNA, 2007, June 8; Gordis, 2005; Govier, 2004; Gyimah, 2006; Hesse, AduAryee, Entsua-Mensah, \& Wu; 2006; JHPIEGO, 2000-2012; Khalil, 2006; KonaduAgyemang, 2000; Konadu-Agyemang \& Shabaya, 2005; Mock, Nyugen, Quansah, Arreola-Risa, Viradia, \& Joshipura, 2006; Munjanja, O. K., Kibuka, S., \& Dovlo, D., 2005; Talley, 2006. 
access local data sources regarding nursing education guidelines, and details that differentiate nurse practice standards for various classifications of nurses. Collaborating in such a manner could afford opportunities to broaden the invisible college of researchers (Patterson \& Russell, 2008).

\section{Developing Awareness of Ghanaian Culture}

Online information resources were examined to better understand societal considerations affecting Ghanaian culture. An in-depth review of institutional, organizational, and news information resources served as the focus for exploration (Anquandah, n.d.; Boateng, 1996-2007; CIA, 2012; Ghana Embassy, 2004-2010; Ghana Tourism, 2007; Ghana West African Missions, n.d.; Library of Congress, African \& Middle Eastern Divisions, Collections and Services Directorate, 2005; Pearson Education, Inc., 2007; Schwimmer, 2007; The Africa Guide, 1996-2008). Findings provided insight to societal factors an outsider may need to consider when exploring the culture of the country. Findings were organized into four broad categories: 1) sociopolitical factors, 2) socio-economic factors, 3) socio-cultural factors, and 4) sociogeographic factors. Related themes of information were grouped under each societal factor category (Figure 2-2). Given the fluid nature of social considerations, factors grouped thematically under one category may likewise fit into another category.

\section{Preliminary Ethnographic Research}

\section{Study Purpose}

In the spring of 2008 I conducted an ethnographic study of nursing and nursing education in Ghana. The purpose of that study was to describe and interpret the cultural contexts that affect nursing practice and nursing education in Ghana so future international nursing education endeavors could be structured in a meaningful way for the setting. Specific aims of the ethnographic study were to examine the environment, social setting, and culture for:

1. The country of Ghana, including regional variations.

2. Professional nursing practice and education in Ghana.

3. Institutions associated with nursing practice and nursing education in Ghana.

\section{Oversight and Access}

The Institutional Review Board (IRB) at The University of Tennessee Health Science Center (UTHSC) provided ethical oversight and study approval for the 


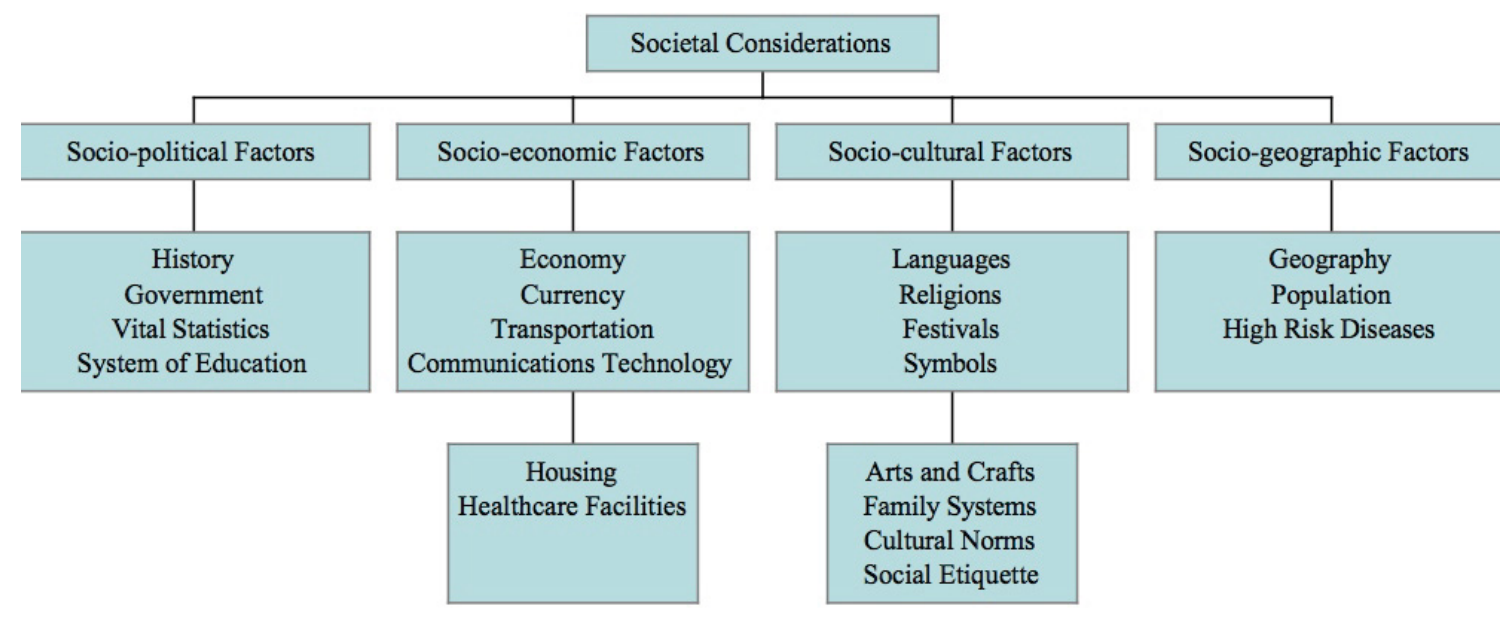

Figure 2-2. Societal factors for consideration when developing cultural awareness.

Note: Factor categories and individual factors where derived from literature reviewed prior to 2008 preliminary research in Ghana.

Sources: Anquandah, n.d.; Boateng, 1996-2007; CIA, 2012; Ghana Embassy, 2004-2010; Ghana Tourism, 2007; Ghana West African Missions, n.d.; Library of Congress, African \& Middle Eastern Divisions, Collections and Services Directorate, 2005; Pearson Education, Inc., 2007; Schwimmer, 2007; Africa Guide, 1996-2008. 
ethnographic study. A personal friend and native Ghanaian served as gatekeeper to data sources in Ghana, and coordinated my transportation and room and board while I was there. A member of the Ghanaian delegation that visited the University of Alabama at Birmingham in the fall of 2007 served as another gatekeeper at their university and affiliated teaching hospital.

\section{Data Sources and Analysis}

Qualitative data sources collected during three weeks of fieldwork in 2008 included semi-structured interviews $(\mathrm{n}=11)$, researcher observations and reflections from daily journal entries, anonymized photographs, and institutional/ organizational documents. Data triangulation included observations and interviews in various regions of Ghana, with fieldwork that enabled visits to 6 of the 10 geographic regions. Interviews were audio-taped and transcribed verbatim in fieldwork format into Microsoft Word. Transcribed data were read repeatedly, and grouped thematically to illuminate the contexts (Patterson \& Russell, 2009).

\section{Ethnographic Findings Organized}

Ghana context. Ethnographic study findings were organized in response to specific aims (Patterson \& Russell, 2009). Concerning the context of Ghana, findings revealed that despite widespread poverty, Ghanaians are a courteous, respectful people who show honor to visitors. Inequities in resource distribution compound the widespread poverty, with inequities evident between urban and rural settings. Geographic inequities in resource distribution were also evident between the northern and southern sections of the country, with more densely populated areas primarily in the south. Higher levels of resource distribution were seen in the more heavily populated urban and southern areas. Each of the 10 geographic regions has its own capital city and regional hospital.

Findings revealed that tribal conflicts occur somewhat frequently in the northern regions of Ghana, where avoiding nighttime travel was advised, as was attention to current socio-political undercurrents and tribal happenings. Language barriers were revealed as a contributing factor to problems with tribal communications. Cultural preparations before beginning the ethnographic study revealed more than 76 ethnic groups and sub-cultures in Ghana, and over 60 tribal languages (Anquandah, 2006; CIA, 2012; Ghana embassy, 2004-2010; Africa Guide, 1996-2008). English was verified as the official language in Ghana. It is mandated for use in schools and used in many businesses in urban settings; however local languages were more commonly used in everyday communications.

Challenges relating to the existing infrastructure in Ghana were identified. Those challenges included: a) limited Internet access, b) poor Internet connectivity, c) limited paved roads and public transportation, d) frequent power outages, and e) lack of potable water. Realities of day-to-day life during fieldwork in Ghana demonstrated that Internet 
access and connectivity were better using a wireless access card during off-peak hours (after 11PM and before 7AM). Power outages occurred frequently in both rural and urban settings during fieldwork experiences. Water for drinking was obtained by boiling water and filtering it before cooling and bottling for consumption, or by purchasing bottled or bagged drinking water. Study findings revealed that, although urban areas have water systems that deliver piped water to residential and commercial buildings that are plumbed, the likelihood of water field contamination is high, and tap water should not be used for drinking. Fieldwork experiences verified open sewers throughout Ghana, often running beside roadways, used as a repository for assorted wastes.

Study findings also revealed that the country of Ghana faces health care challenges that are related to the use of traditional versus modern medicine. Rural - urban healthcare resource distribution inequities compound the challenge, with more rural areas often relying on traditional practitioners or healers as their primary source of healthcare. Findings indicated that modern medicine had often been the last resort for many Ghanaians, attempted only after traditional practices had failed to alleviate the problem, and many times after it was too late to make a difference.

Professional context. Concerning the professional context for nursing practice and nursing education in Ghana, the ethnographic study revealed an extreme shortage of registered nurses (Patterson \& Russell, 2009). Findings also revealed that existing nurses have demonstrated ingenuity and innovativeness in response to the scarce resources available for health care. Study participants indicated that there had been little oversight of nursing practice in rural health care settings, owing to the scarcity of $\mathrm{HRH}$.

Study findings revealed socio-economic factors influencing nursing education and nursing practice in Ghana. Government provided higher education for nursing education requires graduates perform a period of national service after graduation. Low wages and undesired placements for national service (often rural areas away from family) have presented barriers to the profession. Limited professional development opportunities, limited workshops for license renewal requirements, and limited numbers of experienced nurses to serve as role models or mentors were also identified as barriers to the profession. Findings indicated that nurse migration had been a challenge for the profession, with nurses leaving the country to seek a better lifestyle, graduate education, or professional advancement.

Compounding the challenges associated with the nursing shortage and nurse migration in Ghana, ethnographic findings indicated a challenge associated with the way the public perceives nurses in Ghana. Several interview participants reported seeing nurses yelling at patients, and verbalized their perceptions that there was a lack of professionalism from nurses in Ghana. Findings suggested that the low public perception of the nursing profession may be linked to a lower score requirement for admission to nursing schools on senior secondary exams versus score requirements for university admission. 
Institutional contexts. Concerning institutional contexts for nursing education and practice in Ghana, findings from the ethnographic study revealed organizational structure for educational oversight, nursing program accreditation, and regulation of nursing practice in Ghana (Patterson \& Russell, 2009). The Nursing and Midwifery Council of Ghana (NMCG) oversees accreditation of nursing programs, nursing curriculum standards, and qualifies those who can sit for licensing exams. Ghana's Ministry of Health (MOH) oversees clinical placement of nursing students, job posting after graduation, and educational programs of nursing students at nursing schools. Ghana's MOE oversees university based nursing education programs.

Study findings also provided insight to low resource realities at institutions associated with nursing education and nursing practice in Ghana. Educational institutions have limited textbooks, computers, teaching supplies, and inadequate numbers of qualified nursing tutors. Hospitals and health care clinics have limited human resources, supplies, equipment, and facilities. Limited public health care resources are progressively scarce according to institutional hierarchy (teaching hospitals $>$ regional hospitals $>$ district hospitals $>$ local clinics). Findings verified the existence of two teaching level hospitals in the entire country of Ghana: one in the capital city of Accra, and the other in the city of Kumasi. Both of the hospitals are located in the southern half of the country.

\section{Conclusions}

Effective efforts to support nursing education needs in low resource countries require consideration of the cultural contexts for nursing. Ethnographic findings concerning cultural contexts affecting nursing education and practice in Ghana (Patterson \& Russell, 2009) were cross - validated through multiple data sources and correlated with

published reports. Study findings revealed nursing education needs for: a) mentoring/ role modeling, b) professional development, c) advanced education, d) leadership development, e) professional workshops, f) supplies/ resources for education and practice, and g) qualified nursing tutors/ faculty members.

\section{Limitations}

Limitations for the ethnographic study included:

- A novice researcher as the principal investigator.

- An unexpected language barrier resulting in slowed understanding during data collection and analysis.

- The researcher as an unknown entity to many key informants.

- Limited access to key informants and key nursing education resources, including an inability to obtain a copy of the national nursing curriculum. 


\section{Future Endeavors}

Findings from the ethnographic study can enhance understanding of the realities of nursing education and practice in Ghana, and facilitate the development of future nursing education partnership efforts. Future endeavors should target identified nursing education needs and plan for study limitations encountered in the ethnographic research (Patterson \& Russell, 2009). Access limitations encountered during the study indicated the need for future research that incorporates insights from key informants and individuals with a working knowledge of the systems regulating nursing education and nursing practice in Ghana.

\section{Researcher Perspectives}

Self-reflection and transparency regarding a researcher's personal perspectives provide insight to professional knowledge, theoretical understandings, past experiences, and the researcher's lens through which the research will be conducted (Corbin \& Strauss, 2008). In grounded theory research, revealing researcher perspectives as background information draws attention to pre-existing knowledge and worldviews, and heightens awareness to personal perspectives as a mechanism of maintaining sensitivity to the data while acknowledging the value of researcher insights during the interpretative process (Corbin \& Strauss, 2008). Self-reflection concerning personal perspectives relevant to this study suggests that I should reveal those experiences, understandings, and existing knowledge bases.

\section{Personal Experiences and Perspectives}

The profession of nursing beckoned to me even as a young child. I am sure the initial attraction stemmed from wanting to imitate my favorite grandmother who was a dedicated registered nurse (RN). She exhibited a pride in her profession and a deep sense of satisfaction in her chosen career that I wished to experience firsthand. I became a third generation RN and have passed the legacy of nursing on to my daughter. My professional goals are the result of long-standing dreams fueled by a lifestyle that is governed by a personal commitment to serve God and humanity. I was raised the daughter of a minister, and married a minister's son. Throughout my life I have been taught to be purposeful and proactive in finding ways to most effectively show love and compassion to the people around us. I have never believed that it was enough to simply enjoy what the world offers; instead, I believe we each have a duty to help address societal needs.

Private life roles of birth mother, foster mother, preacher's daughter, and elder's wife have each helped me to see people as individuals with unique needs that are relative to their own life experiences. Long-standing friendships and on-going relationships with missionaries in various parts of the world have increased my awareness of global health concerns and existing inequities, while challenging me to envision ways I, as a nurse educator and doctoral candidate, can help. 


\section{Professional Perspective}

Shaped by nursing values and educational experiences. My personal worldview has also been shaped by the core values that guide professional nursing practice in the United States (US). Those values include social justice, human dignity, autonomy, integrity, and altruism (American Association of Colleges of Nursing, 2008). Thirty plus years of nursing practice, six years as a nurse educator, three years involvement with a project to improve nurses' information literacy competencies, and five years of doctoral studies combined to form the knowledge base and professional perspective with which I approached the research.

Doctoral studies have broadened my understanding of international nursing education endeavors, global health needs, health disparities, and organizational resources that target global health concerns. Cultural awareness preparation and knowledge acquisition before traveling to Ghana included in-depth literature reviews and online research to gain information regarding cultural contexts, nursing education and practice, healthcare, and health disparities in Ghana. An issue I encountered during cultural awareness preparation involved locating and discerning reliable up-to-date information resources.

Shaped by preliminary research experiences. Preliminary research in Ghana helped shape my perspective of nursing education needs there. Personal experiences during ethnographic research in Ghana illuminated the need for outsider training and education that can address healthcare needs and related issues unfamiliar to most US nurses, such as malaria, guinea worm, tropical diseases, and folk medicine. First-hand experience reinforced the need for two-way exchange of information in international nursing education endeavors. Personal interactions in Ghana led to the realization that there is much that US nurses can learn from their counterparts in low resource country settings.

Cultural awareness continued during ethnographic fieldwork in Ghana, where the realities of life increased personal understandings while stretching my own worldviews at times including aspects of cultural shock. Issues arose that advance planning had not foreseen, illustrating the need for flexibility while honoring and respecting different worldviews. A street scene was observed where a man was being severely beaten by a crowd - apparently because he had stolen something. My Ghanaian guide and driver stated that this practice was common. A UN news report published after my return to the US (IRIN, June 23, 2008) validated this concept, noting the presence of vigilante groups that defend the peace in Ghana, while sometimes inappropriately beating innocent people. Other safety issues were realized while I was traveling under Ghanaian escort to Northern Ghana. I was taking photographs of a scene in the distance while parked along a rural roadside when local men angrily approached my Ghanaian escort. Language barriers prohibited my compete understanding of the verbal exchange, but safety and isolation were major concerns. Instructions from my guide had indicated that taking photographs from the vehicle at a distance was permitted - but those men resented the action. The incident broadened personal understandings of regional cultural variations 
associated with subcultures and ethnic groups in Ghana. Ghanaian contacts provided examples of tribal conflict and unrest in the Northern part of Ghana, illuminating the need for daylight travel and peaceful encounters. Another UN news report, also published after my return home, drew attention to continued tribal conflict in Northern Ghana (IRIN, June 27, 2008).

\section{Theoretical Understandings}

Theories that have shaped my professional and personal worldview include concepts related to teaching and learning, research methodologies, and information literacy. Foundational principles related to the processes of teaching and learning suggested that defining characteristics pertaining to the curriculum, educators, and students should be assessed to enable effective teaching and learning strategies that could address learning needs (Billings \& Halstead, 1998). Understandings relating to grounded theory methodology prompted me as the researcher, to strive for continued awareness to personal perspectives, use those perspectives as a source for ongoing sensitivity and insight to the data, and record related thought processes during self-reflection, data collection, and analysis (Corbin \& Strauss, 2008).

Theoretical understandings arising from professional experiences associated with teaching strategies to promote information literacy for evidence based nursing practice have also influenced this grounded theory research. During participation in the LISTEN Project (UTHSC, 2007-2010) my team defined information literacy "as a 3-fold acquisition of attitudes, knowledge and skills in a 5-step information process: needing, seeking, accessing, evaluating, and applying information" (Patterson, Carter-Templeton, \& Russell, 2009). This grounded theory dissertation research sought to identify the learning and information processes outsiders have undergone while developing the knowledge base to guide nursing education practices in unfamiliar settings.

\section{Summary}

Limited resources are available to guide nurse educators through the process of developing the necessary knowledge base for role performance in an unfamiliar setting (Haussler, 2006; Lange \& Ailinger, 2001). MDGS have re-focused developmental aid toward capacity building and mutually beneficial, equitable, partnership roles (King, 2009; Wright, Zerbe, \& Korniewicz, 2001). Research is needed to explore the processes inherent with nurse educators learning what they need to know to fulfill their role in an unfamiliar setting, with consideration to existing global health contexts. Cultural awareness of the research setting was raised through literature reviews that explored health disparities and country specific cultural considerations. Preliminary ethnographic research revealed difficulty accessing needed information, and indicated a need for insider knowledge to facilitate outsider understandings. Researcher perspectives provided insight to existing worldviews, and fostered ongoing reflection and researcher transparency to support data sensitivity throughout this grounded theory research. 


\section{CHAPTER 3. METHODOLOGY}

This chapter describes the research methods and procedures used in this study. First, a brief overview of the study's methodology is presented. Next, rationale for the selection of the specified unfamiliar setting are presented, followed by details concerning human subjects protection, sampling, collection tools and instrumentation, methodological rigor and trustworthiness, and lastly, methodological procedures.

\section{Grounded Theory Overview}

Grounded theory was introduced as a methodological approach for qualitative research through the work of Glaser and Strauss (1967). Variations exist in methodological techniques for grounded theory (Chen \& Boore, 2009; Cutcliffe, 2000), in part due to individual perspectives and constantly expanding bodies of evidence related to the method (Corbin \& Strauss, 2008). Procedures described by Corbin and Strauss (2008) guided the study methodology for this research. Their $20083^{\text {rd }}$ edition publication presents information based on the joint work of Corbin and Strauss (Strauss died in 1996), and introduces Corbin's self-defined more open analytical processes. She defined grounded theory analysis as "a researcher-driven thinking and feeling process" (p. xi). The method provides a way to build theory from data, through an exploration of a social phenomenon and its dimensional concepts, in an interpretative analytic process that develops empirical knowledge and facilitates a shared understanding between colleagues (Corbin \& Strauss, 2008). Grounded theory methodology is a good fit for healthcare education research that examines teaching and learning processes in the context of their socialization (Tavakol, Torabi, \& Zeinaloo, 2006). This research explored the tacit knowledge of individuals experienced in partnering for nursing education in an unfamiliar setting. Concepts arising from this study's data were used to develop a theoretical model to reveal the learning processes demonstrated by outsider nurse educators when preparing to assume the nurse educator role in an unfamiliar setting. By revealing the tacit knowledge of insiders, this grounded theory research makes that knowledge explicit, thereby facilitating an enhanced understanding and shared professional knowledge among the global community of nurse educators.

\section{Identification of the Unfamiliar Setting}

Although the study problem, purpose, specific aims, and research question could be applicable to virtually any nursing education partnership in an unfamiliar setting, Ghana, Africa was chosen as the unfamiliar context for this study for the following reasons:

1. The site meets the needs of the research problem and study purpose. 
2. Ghana is considered a "prime candidate [for scaled up foreign aid in] the achievement of MDGS" (WBG, 2010).

3. I have long-standing personal faith-based contacts in Ghana, with requests dating back more than 15 years to come to Ghana and help with health-related needs and nursing education efforts.

4. In the fall of 2007 I met with members of a delegation from a university in Ghana when they visited the University of Alabama at Birmingham School of Nursing and its' affiliated WHO Collaborating Center for International Nursing, and requested help to supplement nursing faculty capabilities at their institution.

5. In 2008, I built on earlier contacts, and conducted preliminary research in Ghana, using an ethnographic design to describe the contexts for nursing education in Ghana.

6. From six years of exploratory research, I have personal understanding of the challenges outsiders can encounter when trying to uncover hidden knowledge that is relevant to outsiders' interpretation of the nurse educator role in Ghana.

\section{Human Subjects Protection}

The UTHSC IRB provided ethical oversight for this grounded theory research and granted exempt status for the research (Appendix A). The IRB waived requirements for study participants to sign a consent form, and approved the use of a consent statement (Appendix B) in lieu of a written record. All individuals asked to participate in the study received a copy of the consent statement that detailed the interview process. The consent statement also specified time costs to participants and confidentiality measures for study records, resources, and reports. At the beginning of each interview, participants were thanked for their willingness to participate. Their receipt of the consent was confirmed (for participants contacted through email and interviewed by phone), and an opportunity to ask questions related to their participation was provided.

During preliminary research in Ghana in 2008, I discovered a different approach to human subject protection for non-interventional educational research in Ghana. In addition to UTHSC IRB requirements, Ghanaian guidelines were followed, in keeping with ethical standards for conducting public health related research in Africa (Erinosho, 2008). Formal letters of intent were presented to governmental organizations associated with nursing education in Ghana requesting their support for this study. Letters of intent were sent by email to my Ghanaian liaison and hand delivered by him during the fall of 2011 to officials at the a) NMCG (Appendix C), b) Ghana MOH (Appendix D), and c) Ghana MOE (Appendix E). Preliminary research in 2008 revealed each of the targeted organizations as key stakeholders for nursing education in Ghana. The inclusion of these stakeholders in the research process was aligned with recommendations from the Global Health Workforce Alliance (GHWA, 2008b) for international partnerships congruent 
with national needs. Ghana designated a 5-year work plan (2007-2011) to tackle the health needs of their population, with health related research a factor in that plan (Ghana $\mathrm{MOH}, 2007)$. As a branch of the MOH, the Ghana Health Service oversees research related to training and capacity building for health (Ghana Health Service, n.d.), in keeping with mechanisms established during the Ghana-Dutch Collaboration (Advisory Council for Scientific Research in Development Problems [ACSRDP], 1998). The letters of intent for the Ghana portion of this study followed the framework of the GhanaianDutch collaborative (ACSRDP, 1998). My Ghanaian liaison indicated through electronic and telecommunications during the fall of 2011 that support and interest in this study had been verbalized by contacts at all three governmental organizations in Ghana.

I arrived in Ghana on Wednesday, February 15, 2012. The next day, through efforts facilitated by my Ghanaian liaison I met with key officials at the MOH, hand delivered another copy of the letter of intent for their organization, and solicited their approval for the research in Ghana. Verbal approval was granted, with instructions to proceed, and an official letter of introduction indicating their approval was promised. I returned to the $\mathrm{MOH}$ three more times before obtaining the actual letter of introduction and approval (Appendix F). Officials at the MOH had provided their business cards with email addresses and phone numbers in case verification of study approval in Ghana was required before the letter was ready for pick up.

\section{Sampling}

Theoretical sampling was used to identify and recruit information-rich interview participants, thereby maximizing the development of theoretical concepts in response to the research question (Corbin \& Strauss, 2008). To address the research questions for this study, theoretical sampling began by contacting individuals from the United States and Canada who had been identified as former partners from nursing education endeavors in Ghana. Their participation was solicited as a mechanism to capture the etic perspective from outsider nurse educators who have gone into unfamiliar settings.

Theoretical sampling then continued in a snowball fashion, incorporating knowledge from interview participants in the identification of other information-rich individuals. Sampling efforts targeted participants with a wide array of experiences, to help deepen understanding of the properties and dimensions of emerging theoretical concepts. After theoretical saturation of the data from North American participants was determined, theoretical sampling transitioned to information-rich informants from within Ghana who were experienced partnering with outsiders for nursing education endeavors in Ghana. Ghanaian participants were specifically targeted to add the emic perspective to research findings in light of insider insights and understandings for issues that outsiders were challenged to understand. Ghanaian participants were identified through researcher insights from preliminary research, literature reviews, and referrals from North American participants concerning key informants inside Ghana. 
For the targeted interview participants, inclusion criteria included the ability to speak and converse in English and a minimum age of 18 years. Sampling continued until theoretical saturation was determined. Corbin and Strauss (2008) defined theoretical saturation as the analysis point at which the researcher determines all theoretical categories are developed to the extent that further data collection would add little to enrich understanding of the dimensional properties of the concepts, with the understanding that variability could always be discovered. As the primary instrument for this grounded theory study, I made the determination of theoretical saturation, with guidance from my dissertation chair. Interviews for this study targeted various partners' insights to add to the dimensional properties of theoretical concepts, and provided an avenue for comparisons and reflexivity based on variations in individual perspectives and contextual understandings. Targeting interview participants from inside and outside of Ghana added to the richness of the data by capturing the emic and etic perspective regarding information to facilitate partnering for nursing education in an unfamiliar setting.

\section{Identification of Target Sample}

Methodological decisions regarding identification of the targeted sample were influenced by the following considerations:

1. Feasibility of conducting interviews to enable the capture of verbal communications either by telephone or face-to-face interviews.

2. Literature evidence of several past nursing education partnerships between individuals and institutions from within North America (US and Canada) and institutions or organizations in Ghana. Published literature, and web resources were reviewed (see Chapter 2) to identify individuals and institutions with firsthand knowledge about partnering for nursing education in Ghana (an unfamiliar setting). North American participants were drawn from nurse educators and nursing program/ organizational administrators who had personal experience functioning as a nurse educator in Ghana. The role in which their experience was manifested was captured in participant demographic information (see Chapter 4).

3. Earlier introductory communications I had with select individuals experienced with nursing education partnerships in Ghana, while I was networking, preparing for, and conducting preliminary research in 2008. The deputy director of a WHO Collaborative Center for International Nursing facilitated my initial contact and referral to nurses with first-hand knowledge of nursing education partnerships in Ghana. Other referrals and contacts were discovered while I was presenting professional information and networking at professional meetings.

4. Common understanding of nursing education standards and guidelines among North American participants from the US, due to accreditation requirements (Commission on Collegiate Nursing Education, 2009), lending support to a 
common language between nurse educator participants and myself as a researcher from the US.

5. Theoretical sampling incorporated insights from interview participants in the identification of other individuals who qualified to participate in the study.

\section{Participant Recruitment Procedures}

Email letters of study introduction were sent to North American individuals initially identified as potential participants, with researcher contact information and plans for follow-up contact. Study introduction letters incorporated an IRB approved initial contact script (Appendix G) that provided a brief explanation of the study, researcher background, including preliminary research and related presentations. Each initial contact scripted letter was personalized to indicate the specific Ghana nursing education endeavor that had led me to contact them. Follow-up contact (Appendix H) was initiated by phone or email, within two weeks of initial contact emails being sent, to identify willing participants and schedule individual interviews.

North American participants were recruited from various locations, institutions, and organizations across the US and Canada during September and October of 2011 to serve as data sources for nurse educator outsider perspectives. In total, study introduction letters for North American participants were sent to 27 individuals during an initial round of recruitment efforts. From those recruitment contacts, 6 individuals did not reply to the initial or follow-up contact efforts, 4 individuals' replies indicated they were ineligible for the study, and 17 individuals expressed interest in participating. From the pool of individuals interested in participating, two were unavailable for interviews until early 2012. One individual withdrew interest in participating relative to faculty workload and competing demands. In total, the pool of qualified individuals available as data sources for North American insider perspectives during the fall of 2011 included 14 potential participants. Referrals from study participants provided a few new contacts who were added to the pool of potential participants for second round recruitment efforts if necessary, but many of the referrals were for individuals already targeted for recruitment in the first round.

Participant recruitment for theoretical sampling in Ghana began by submitting written letters of intent to key informants at Ghana's MOH, MOE, and the NMCG. I followed up in person upon my arrival in Ghana in February 2012, and met with officials from each of the organizations, soliciting their support of the research. I met with officials at the $\mathrm{MOH}$ on my second day in Ghana, and obtained their support and approval for conducting the research in Ghana. After asking how they could help, $\mathrm{MOH}$ officials provided a typed list of 12 names, positions, and contact information for Ghanaian insiders who had first-hand experience partnering with outsiders from North America for nursing education endeavors in Ghana. 
Before leaving for Ghana, I used contact information harvested from the Global Alliance for Nursing and Midwifery listserv and sent personalized initial email contact letters to two of the individuals whose names I later found were on the $\mathrm{MOH}$ list. Before I arrived in Ghana, one Ghanaian nurse educator had already agreed to participate in this study. I purposefully sought out Ghanaian insiders with knowledge of more than one nursing education partnership experience in Ghana.

Potential data sources for insider perspectives in Ghana included individuals from the academic community who were previously involved in nursing education partnerships with outsiders (nursing tutors and nursing program administrators), and individuals from governmental bodies associated with nursing in Ghana (MOH, MOE, NMCG). In addition to the names provided by the $\mathrm{MOH}$, I acquired contact information for 3 other individuals who had first-hand experience with multiple nursing education partnership experiences, which brought the pool of potential participants in Ghana to 15 individuals.

Potential Ghanaian participants were contacted by telephone, with the assistance of my in-country liaison and host. After briefly explaining the study over the phone, I arranged face-to-face meetings to conduct the Ghanaian interviews. Geographic location of potential participants was a factor while I determined which individuals to contact first in Ghana, as was the diversity of their partnership experiences. I began by contacting select individuals located within an hour's drive of Accra, those that I could feasibly meet given the time restrictions of the fieldwork, and availability of Ghanaian transport. I specifically targeted insiders with multiple, diverse partnership experiences. All of the individuals I approached in Ghana about participating in this research agreed to participate.

\section{Access and Support System in Ghana}

Entry to the Ghana context was facilitated by personal contacts. A US-educated Ghanaian educational doctorate recipient continued to serve as my gatekeeper (a role he assumed during my preliminary research in 2008), and in-country liaison. This gatekeeper's experiences as a youth in Ghana, as an undergraduate university student in the US, and again as a US institution's graduate student living at a distance in Ghana, uniquely equipped him with first-hand knowledge of the realities of education in both countries. He was recently involved in a collaborative effort between Ghana and the Tennessee Board of Regents (TBR, 2009-2010) with distance education and faculty exchange endeavors that targeted the improvement of teaching in Ghana. Since my preliminary research in 2008, he had been added to educational committees in Ghana that opened additional doors for the purposes of this research that were previously not available. Those doors included committee contacts with members of the NMCG, and the collaborative relationship with the MOE and TBR. The fact that the gatekeeper is not a nurse, or a member of the healthcare professions, could have presented a limitation to his role in this study; however, his familiarity with Ghanaian officials at the MOH, NMCG, and MOE seemed to counteract that potential limitation. The fact that the gatekeeper is a male, in a study that focuses on nursing (a profession that is predominately female), 
suggested that gender might have been a consideration. Researcher observations during fieldwork revealed multiple men as high-ranking nursing officers at the $\mathrm{MOH}$ and the NMCG, and prompted awareness to Ghana's ongoing pursuit of gender equality. The current registrar for the NMCG is a female, and the six Ghanaian interviews were equally divided between genders. In the context of the capital city were fieldwork occurred, the gatekeeper's gender might have actually been an advantage, in consideration to cultural interpretations of his authority and prominence.

The accidental death in 2011, of the Canadian expatriate nurse $(14+$ years living in Ghana) who acted as my hostess during my 2008 Ghana research, and had planned to serve as my hostess and peer de-briefer during this study's fieldwork, required adaptation of plans for this study. My doctoral program director from UTHSC served as faculty debriefer during fieldwork, via email and text communications. My in-country liaison and his family graciously provided my room and board, escort, driver, and translator services as needed during fieldwork. He and his wife (an American expatriate) served as informants and peer de-briefers, and helped provide clarity for contextual and cultural considerations while I was in Ghana. As a US citizen living in Ghana, my liaison's wife had first-hand experience with the culture shock that outsiders experience when first going into that setting.

\section{Data Collection Tools and Instrumentation}

Corbin and Strauss (2008) identified a researcher's mind and intuition as the primary tools for grounded theory research. A variety of analytical thought processes (Corbin \& Strauss, 2008) served as a pool of analytic tools for this study, including: brainstorming, asking questions, using constant comparisons, drawing theoretical comparisons, considering various meanings or alternative interpretations, flip-flopping a concept, using personal experience to illuminate meaning, recognizing bias, listening to participants' language, drawing insight from emotional cues, using metaphors, and maintaining sensitivity to the data. In grounded theory, analytic thought processes "...are heuristic devices that promote interaction between the analyst and the data..." (Corbin \& Strauss, 2008, p.85) thereby illuminating possible meanings.

Tangible tools and instrumentation to facilitate collection of data for this study included: a broad interview guide (Appendix I), methodological notes and memos, reflexive journal entries, fieldnotes, interview transcriptions, lists of concepts and codes, matrix illustrations representing potential contextual conditions, and graphic illustrations of the emerging theoretical model. Participant demographics were captured at the beginning of each recorded interview, and not recorded on a separate data collection tool. Demographic questions have been defined in the broad interview guide (Appendix I). 


\section{Methodological Rigor and Trustworthiness}

Mechanisms to address methodological rigor and trustworthiness for qualitative study findings were built into the study design for this grounded theory research. Lincoln and Guba (1985) identified credibility, transferability, dependability, and confirmability as the criteria by which qualitative studies could be evaluated for methodological rigor. Procedural measures to enhance rigor for this study included the use of an audit trail, transcription member checks, peer debriefing, reflexive journaling, constant comparison, prolonged engagement, analytic memos, methodological notes, and faculty oversight throughout data collection, analysis, and interpretation of findings.

\section{Credibility}

Concerning credibility of study findings and researcher interpretations as a human instrument for data collection, I strove for sensitivity to the data during data collection and interpretation (Corbin \& Strauss, 2008). I endeavored to exhibit researcher transparency by consciously acknowledging my own worldviews and individual perspectives (see Chapter 2). I took those worldviews and perspectives into consideration throughout the process of data collection and interpretation, having drawn upon personal experiences and my nurse educator role to stimulate questions and thought processes regarding theoretical concepts as they emerged. To further address credibility and dependability limitations, my faculty chair and the director of my doctoral program have each served as peer reviewers at various points during (Lincoln \& Guba, 1985; McBrien, 2008; Stake, 2010) study design, data collection, analysis, and reporting. Fieldnotes, journal entries, memos, and diagrams have served as a record of reflexivity (Corbin \& Strauss, 2008; McBrien, 2008) and thoughts related to investigator interpretations and analytical reasoning. Research experience during preliminary fieldwork provided firsthand knowledge and skills acquisition for qualitative data collection and analysis involving interviews and reflexive journaling. Participant verification of interview transcripts was offered to interviewees as a mechanism to confirm the accuracy of transcriptions. Researcher solicited informal member checks (Lincoln \& Guba, 1985) were part of the interview process and constant comparative reasoning processes for this study. Verification of researcher interpretations was frequently sought during interviews, by repeating information back to participants as a mechanism to clarify understanding of data pieces.

Mechanisms to address credibility regarding the potential for interviewee response bias included triangulating multiple sources of interview data (via theoretical sampling drawn from outsider [US] nursing education partners and insider [Ghanaian] nursing education partners, including affiliated partners from regulatory bodies for nursing education in Ghana). Constant comparative analysis methods were used from the onset of data collection (Corbin \& Strauss, 2008). Mechanisms to address credibility relating to language limitations for this study included active reflection and clarification of meaning during the interview process (Corbin \& Strauss, 2008; McBrien, 2008), the use of one experienced translator/ interpreter to facilitate consistency and accuracy in 
interpretation (Squires, 2008), reflexivity and disclosure of methodology relating to language limitations and accuracy of interpretations (Squires, 2008), field transcription of Ghanaian interviews by an experienced transcriber with attention to ethics of translation and transcription (Squires, 2008), and member checking (Lincoln \& Guba, 1985; McBrien, 2008; Stake, 2010) to solicit respondents' clarification of researcher interpretations during interviews.

\section{Transferability, Dependability, and Confirmability}

Transferability of study findings to other contexts are addressed through contextual description of theoretical concepts, and a detailed accounting of study data to allow future users of the data to make their own interpretations regarding appropriateness of using the findings to guide research or practice in other settings (Lincoln \& Guba, 1985). In keeping with the constant comparative method inherent in grounded theory research (Corbin \& Strauss, 2008), future users of this grounded theory would need to assess the theory's fit to the potential context in order to determine the appropriateness of testing the theory in that setting. Dependability and confirmability for this study have been addressed by using an audit trail to track the inquiry process and the research product (Lincoln \& Guba, 1985; McBrien, 2008), in an adaptation of the process developed by Schwandt and Halpern (1988). The audit trail organized the research product for this study by tracking a) raw data (interview recordings and transcripts, participant reviews of transcripts, researcher notes, observations, and reflections), b) data reduction (summary notes after interviews, categorical units, TAMS Analyzer analysis summaries), and c) data reconstruction (categorical relationships, coding definitions, properties and dimensions of categories, insights to emergent theory, final theory, and report of findings). Likewise, the audit trail afforded opportunity to track the inquiry process via a) methodological notes (researcher memos relating to thought processes, analysis procedures, coding and data reconstruction decisions, and mechanisms to address trustworthiness and the audit trail itself), b) notes regarding researcher focus (problem and purpose, background understandings, reflexive thoughts and motivations), and c) notes relating to tools and instruments used during the research (central research question, broad interview guide [Appendix I], study procedures/ IRB approved process, recording tools, analysis tools/ software, reference list, and networking list).

\section{Methodological Rigor for Study Limitations}

Other mechanisms to address specific study limitations further enhanced trustworthiness. Triangulating multiple sources of interview data helped address the potential for interviewee response bias, and added rigor to the study design. Using an experienced gatekeeper/ in-country liaison that possessed an understanding of the organizational structure of healthcare and governmental oversight for healthcare in Ghana, and using Ghanaian transcribers for initial transcription of Ghanaian interviews helped address language barriers and enhance trustworthiness for this study. Lastly, issues related to feasibility and limitations related to interview variability were addressed in the data collection plans, by using a broad interview guide and establishing plans to 
inform all participants of the interview agenda and study expectations, regardless of interview format.

Fieldwork for this study was limited to 12 days in Ghana, and accommodated the study purpose and research methodology. Crossley and Vulliamy (1997) noted that condensed fieldwork is appropriate in the context of low-resource countries when a researcher seeks an in-depth understanding of a limited theme, and uses a methodology that supports the scope and purpose of the study. For the purposes of this study, I addressed the limited theme of identifying insider knowledge that can facilitate an outside nurse educator's understanding of the learning processes inherent with equipping oneself for the nurse educator role in Ghana, by using a methodology geared toward enhancing understanding and making knowledge explicit (Corbin \& Strauss, 2008).

\section{Methodological Procedures}

This section provides an organized explanation of study procedures. Data sources are delineated first, followed by data collection for interview data. Procedures for storing and transcribing interview data are presented next, followed by data collection and storage procedures for researcher generated data. Methodological procedures for data management and analyses are then presented, followed by mechanisms to support methodological rigor. Finally, a graphic timeline depicting study procedures is presented.

\section{Data Sources}

Insider perspectives, researcher observations, interpretations, and reflections comprised this study's qualitative data sources. Individuals identified for theoretical sampling comprised the data sources for insider perspectives. Researcher generated data sources included analytic memos, methodological notes, a running list of emerging concepts and codes, reflexive journal entries, and illustrations that helped map emerging awareness to concepts and their hierarchical relationships

Data sources for insider perspectives were the interview participants themselves. There were a total of 16 individuals who served as data sources for insider perspectives. Individuals from North America $(n=10)$ provided data depicting the perspective of nurse educator insiders with experience going into an unfamiliar setting; and individuals from Ghana $(n=6)$ provided data depicting the perspective of Ghanaian insiders with experience working with outsiders for nursing education in Ghana. The two groups of participants provided opportunity to capture insider perspectives from both sides of the partnership experience, and afforded insights that might otherwise have been missed if only one side were examined. Demographics of the interview participants can be found in Chapter 4. 


\section{Data Collection for Interview Data}

Individual interviews were conducted face-to-face or via phone so verbal responses could be recorded. Selection of interview medium was dependent on feasibility. Research from a related field provided evidence of the fit for telephone interviewing techniques during grounded theory research that explored physical therapy education programs involved with international teaching and learning experiences (Pechak \& Thompson, 2009). Telephone interviews have been deemed appropriate for research involving participants who are geographically distant from the researcher (King \& Horrocks, 2010; Novick, 2008; Smith, 2005), and especially relevant to international research (King \& Horricks, 2010). Relative to geographic distribution, the ten North American interviews took place by phone. Fieldwork in Ghana enabled face-to-face interviews for those six participants.

Interviews were conducted using a Broad Interview Guide (Appendix I). Telephone interview procedures were guided by recommendations for qualitative data collection by phone (Burke \& Miller, 2001; King \& Horrocks, 2010). Advance preparation for telephone interviews involved piloting the telephone recording process (Burke \& Miller, 2001; King \& Horrocks, 2010) with family members in other cities, as a mechanism to becoming familiar with the recording equipment before initiating study interviews. In addition, the first six North American study contacts (first contacted by email on September 20,2011) provided opportunity to pilot (1) initiating email contact scripts, (2) follow-up contact scripts, (3) scheduling of interviews, (4) briefing potential participants on expectations and the consent process, (5) confirming consent and confidentiality, (6) collecting participant demographics, and (7) using the Broad Interview Guide to conduct interviews.

Communications with all participants (telephone and face-to-face) included the provision of the Broad Interview Guide (as an interview agenda), informed consent statement, and interview schedule (Burke \& Miller, 2001; King \& Horrocks, 2010), as mechanisms to facilitate a focused interview geared to the study purpose. During the interview process, the broad interview guide was used to focus the interview while soliciting individual experiences. Conscious efforts were made to not impose preconceived ideas on the emerging data, but to let the interview participants present their understanding of the phenomenon. Probing questions were used to expand on concepts as necessary to facilitate researcher understanding. Constant comparison was used across interviews, and served to guide researcher probes while adding dimensional properties to emerging concepts. Back-up questions were used as needed to probe and stimulate participants to tell their own story as recommended by Corbin and Strauss (2008) for grounded theory research. Examples of probing questions are listed in the Broad Interview Guide (Appendix I).

North American interviews. Data collection began by interviewing ten individuals from across the US that had experience with nursing education partnerships in Ghana, Africa, using the research question and specific aims as a broad interview guide (Appendix I). Interviews began on October 13, 2011, with referral requests to 
participants providing opportunity for snowball sampling to add to the pool of potential participants for theoretical sampling. The final US interview took place on November 8, 2011, when it was determined that data saturation for outsider participants had been reached. Constant comparison of emerging concepts and researcher reflections after each interview suggested theoretical saturation of US interview data after eight interviews. Two additional US interviews were completed to ensure data saturation (when no new categories emerged). A total of 10 interviews were conducted with individuals from across the US. The two individuals from the pool of potential participants who were unavailable for interviews until early 2012 were the only individuals from Canada who expressed an interest in participating. By the time those individuals were available the North American data collection was over. Throughout the remainder of this report the term US participants has been used to identify outsiders who participated in this study.

Ghanaian interviews. Theoretical sampling continued via six information-rich informants from within Ghana who possessed insider knowledge, as identified through researcher experiences and other interviewees, regarding partnering with outsiders for nursing education in Ghana. Insights gained through comparison of US participant responses helped guide question probes for Ghanaian participants. While conducting the first Ghanaian interview on February 20, 2012, I realized that the question probes provided as examples (and not self limiting) on the broad interview guide were geared primarily to the US participants, and needed adaptation to help focus Ghanaian participants to the research intent. I refocused question probes for Ghanaian participants by seeking clarification of information and issues that US participants had not understood. I also used probes to solicit Ghanaian insights as to what they thought an outsider should know in order to enact the nurse educator role effectively in their country. Research for this study during fieldwork in Ghana (February 15-25, 2012) began at the $\mathrm{MOH}$, and progressed quickly to participant recruitment and data collection, aided by the official approval and support of the Ministry. In total, six interviews were conducted in Ghana during the week of February 20-24, 2012. Feasibility limitations that affected sampling methods and data saturation for the Ghanaian participants are discussed in Chapter 5.

\section{Procedures for Storing and Transcribing Interview Data}

Data storage. Hard copy and electronic data sources for the study were maintained in a secure location, along with all interview recordings, journals, computer records, and study documents. During Ghanaian fieldwork, a briefcase dedicated to that purpose was kept in a locked cabinet when not in use. Interview recordings were backed up digitally on compact disc and jump drive on the evening of their occurrence while in Ghana. Computer and jump drive access were password protected. To facilitate data integrity while abroad, digital recordings were uploaded to a secure password protected Internet cloud storage site as a safeguard against airline travel and the potential for damage to digital recordings during transit. 
Transcription and verification process. Interviews were audio-recorded, and transcribed into Microsoft Word as soon as possible after each interview. Individual interviews were limited to 60 minutes. Researcher notes for each completed interview were reviewed before conducting a new interview, to provide insight to recurring themes, potential probing questions, and monitor for data saturation. I personally transcribed interviews with all of the US participants before traveling to Ghana. To address the accuracy of interview data, participant verification served as a mechanism to validate the transcriptions via email communications after each interview was transcribed. All US participants were sent electronic copies of their individual transcripts. Two of the ten US participants responded to an initial verification request email and validated the accuracy of their interview transcripts. One of those two requested a few lines of that transcript be deleted and classified as off the record. A follow-up request email was sent in June 2012, to the remaining eight US participants, as a safeguard against lost or forgotten email communications. A $60 \%$ response rate was achieved with US participants when four of the remaining eight participants validated their interview transcripts.

Special considerations for Ghanaian transcription. Challenges I experienced while transcribing interviews during preliminary research in 2008 (relative to strong Ghanaian accent and variations in language terminology) prompted a plan to hire Ghanaian transcribers for this study. Original plans were to have each interview transcribed while I was in the field. I was reliant on my in-country liaison/gatekeeper to facilitate transcription of Ghana interviews. Challenges arose related to the setting, including different interpretations of time, the limited timeframe for my fieldwork, and competing responsibilities for the pre-arranged transcriptionist. I left Ghana without any transcriptions completed, but with a back-up transcriptionist in place. Revised transcription plans were further challenged when I discovered the back-up transcriptionist had contacted typhoid fever the week after I left Ghana. Transcription plans were revised again, to use transcriptionist services from both individuals to complete initial transcriptions. Following transcription of Ghanaian interviews in Ghana, I personally reviewed the taped interviews against the transcribed records and essentially retranscribed each interview to correct inaccuracies or missing data. Verification of interview content was offered to each Ghanaian participant, via email communications with attached interview transcript. Two of the six Ghanaian participants reviewed and edited their transcripts for clarity of understanding. Overall, this study had a 50\% response rate for checking accuracy of interview transcripts ( 8 of 16 participants validated their transcripts).

To promote confidentiality of interviews, Ghanaian transcribers were solicited from sources unrelated to the study. Ethics of research were discussed with the transcribers, and they were asked to maintain confidentiality of study participants and study data. Following transcription, transmission to the researcher, and verification of researcher receipt of each interview transcript, each transcriptionist was instructed to destroy all electronic and hard copies of individual interviews and all recordings for this research. After discussions with my Ghanaian gatekeeper the decision was made to use a confidentiality statement for hired transcribers instead of a signed confidentiality form, as a mechanism to avoid misunderstandings and a lack of trust that could undermine the 
research effort. Both Ghanaian transcriptionists received a copy of the Transcriptionist Confidentiality Statement (Appendix J).

\section{Data Collection and Storage for Researcher Generated Data}

Researcher observations of anonymized behaviors; activities, conversations, interactions, processes, and experiences (including the context of occurrences) were recorded by the researcher in journal entries and transcribed in fieldnote format into Microsoft Word as soon as possible. Reflective thought processes of the researcher, including individual perspectives, interpretations, and feelings, were recorded in memo format, transcribed and stored like the observational data. Methodological decisions were recorded in methodological notes, transcribed, and stored as other data. US interviews were transcribed into Microsoft Word in fieldnote format, and coded line by line for first cycle process coding and in vivo coding. First-cycle process codes were numbered and recorded in track changes comments, and in vivo codes were highlighted/ marked in text. Ghanaian interviews likewise underwent first-cycle process and in-vivo coding, after researcher review and conversion to fieldnote format. A running list of emerging concepts and process codes was developed and maintained in Microsoft Word. At the end of first cycle coding there were 1672 pieces of process-coded data related to emerging concepts, and 140 coded pieces of data that defined participant demographics.

Data collection was bounded by the problem focus and purpose of the study. During US data collection, email, text, and phone communications with my dissertation chair, and $\mathrm{PhD}$ program director served as evidence of faculty oversight, guidance, and feedback. During Ghanaian fieldwork, text messaging served as an effective means of communicating with faculty, without any of the delays encountered with Internet access there. While in Ghana, credibility of findings was enhanced through peer debriefing sessions with the gatekeeper and in-country liaison. He sat in on the first and last interviews, and was able to discuss responses with me, lending insight to cultural nuances and pointing out contextual variations. Debriefing sessions with him and electronic communications with faculty members served as opportunities for me to explore understandings and interpretations of unfolding data while testing ideas for the emerging design (Lincoln \& Guba, 1985).

\section{Data Management and Analysis}

Data were organized using a formalized plan for data collection and management guided by Corbin and Strauss (2008) grounded theory methodological procedures. The plan included itemization of data sources and documents in an audit trail for naturalistic inquiry as recommended by Lincoln and Guba (1985). The audit trail was adapted from the process set forth by Schwandt and Halpern (1988) and described earlier in this chapter (see Transferability, Dependability, and Confirmability). 
The TAMSAnalyzer freeware software program, developed by qualitative social researcher Matthew Weinstein (MacResearchSW, 2007) helped organize and analyze the qualitative data for this study, beginning with second cycle coding. Other researcher generated data sources contributing to the analytic process were likewise stored and managed. Methodological notes and reflexive memos were added to the TAMS Analyzer data management program for this study. Data management included an inductive ongoing data analysis process using constant comparison as data were collected. The first interview signaled the start of analysis, as soon as I began to logically think about the data. Thinking strategies (Corbin \& Strauss, 2008) were focused on emerging concepts and pattern recognition, with reflective consideration to researcher and participant theoretical frameworks, value systems, paradigms, and pedagogical perspectives as interpretations were sought. I strove to maintain a commitment to data sensitivity (Corbin \& Strauss, 2008) throughout the process of exploring, coding, and interpreting the data. Data were interpreted with a focus on the realities of the phenomenon, using participants' words for in-vivo coding (Corbin \& Strauss, 2008) as applicable to enhance understanding of nursing education in unfamiliar settings.

Sequential but overlapping steps in this grounded theory analysis process ran concurrent with data collection and management based on Corbin and Strauss (2008) methodological procedures, blended with coding guidelines for grounded theory research (Saldana, 2009), in an ongoing exploration of preliminary findings. During analysis, the researcher:

1. Thought about and made sense of pieces of data, using numbered and dated analytical memos as a data source and record of thoughts across the research continuum of explaining and coding concepts.

2. Assigned first-cycle initial (open) process and in-vivo codes to concepts identified in the data.

3. Incorporated methodological notes as a data source and record of researcher thoughts about how pieces of data fit together, in awareness to emerging patterns and hierarchy of concepts.

4. Kept a running list of concepts and their codes.

5. Reorganized codes to better define patterns and concepts that emerged from the data by examining first-cycle codes and raw interview transcripts for common threads.

6. Assigned second-cycle, focused codes that identified relationships between categories and sub-categories of concepts, and second-cycle axial codes that indicated their properties and dimensions. 
7. Used constant comparative thought processes to continually examine and reexamine data pieces, concepts, and codes, adding to the properties and dimensions of each concept and code.

8. Included context as part of a consequential matrix that thought about and explored actions and interactions that could affect participant experiences.

9. Sifted and sorted through the data to conceptually organize and structure the analytic process through pattern recognition and graphic illustration of emerging concepts and theoretical understandings.

10. Continued theoretical sampling until theoretical saturation was recognized (when no new categories emerged and the dimensional properties of concepts were fully developed).

11. Integrated concepts and relationships around an overarching, central category, (theoretical coding) searched for missing links or incompleteness, mapped and reworked the analysis as needed until a logical, explanatory theory emerged that intuitively felt right.

12. Made a conscious effort to not allow preconceptions based on literature reviewed to bias the grounded theory process.

A timeline of study procedures from IRB application to conceptualization and emergence of theory has been presented in Figure 3-1. The theory that emerged from this study was grounded in the experiences and tacit knowledge of individuals with firsthand understanding of nursing education partnerships in unfamiliar settings. As such, the theory can guide individual nurse educators through the process of acquiring information foundational to beginning a partnership for nursing education in an unfamiliar setting. The theory identified information past participants needed to enable interpretative understanding of nursing education programs in an unfamiliar setting so educational interventions could be structured to meet contextual needs. This study's findings and theoretical understandings are compared and contrasted against existing literature in the discussion section of this dissertation (see Chapter 5).

\section{Summary}

This research used grounded theory methodology to explore the experiences of individuals with first-hand knowledge about the phenomenon of nursing education partnerships in an unfamiliar setting, and generated a theoretical model to transform insider's relational tacit knowledge about the phenomenon into explicit shared professional knowledge. As the researcher, I served as the primary instrument for this grounded theory research. Data were derived from interviews, researcher observations, reflections, and analytical interpretations. Initial interviews targeted former outside partners for nursing education in Ghana, and solicited their experiences acquiring the 


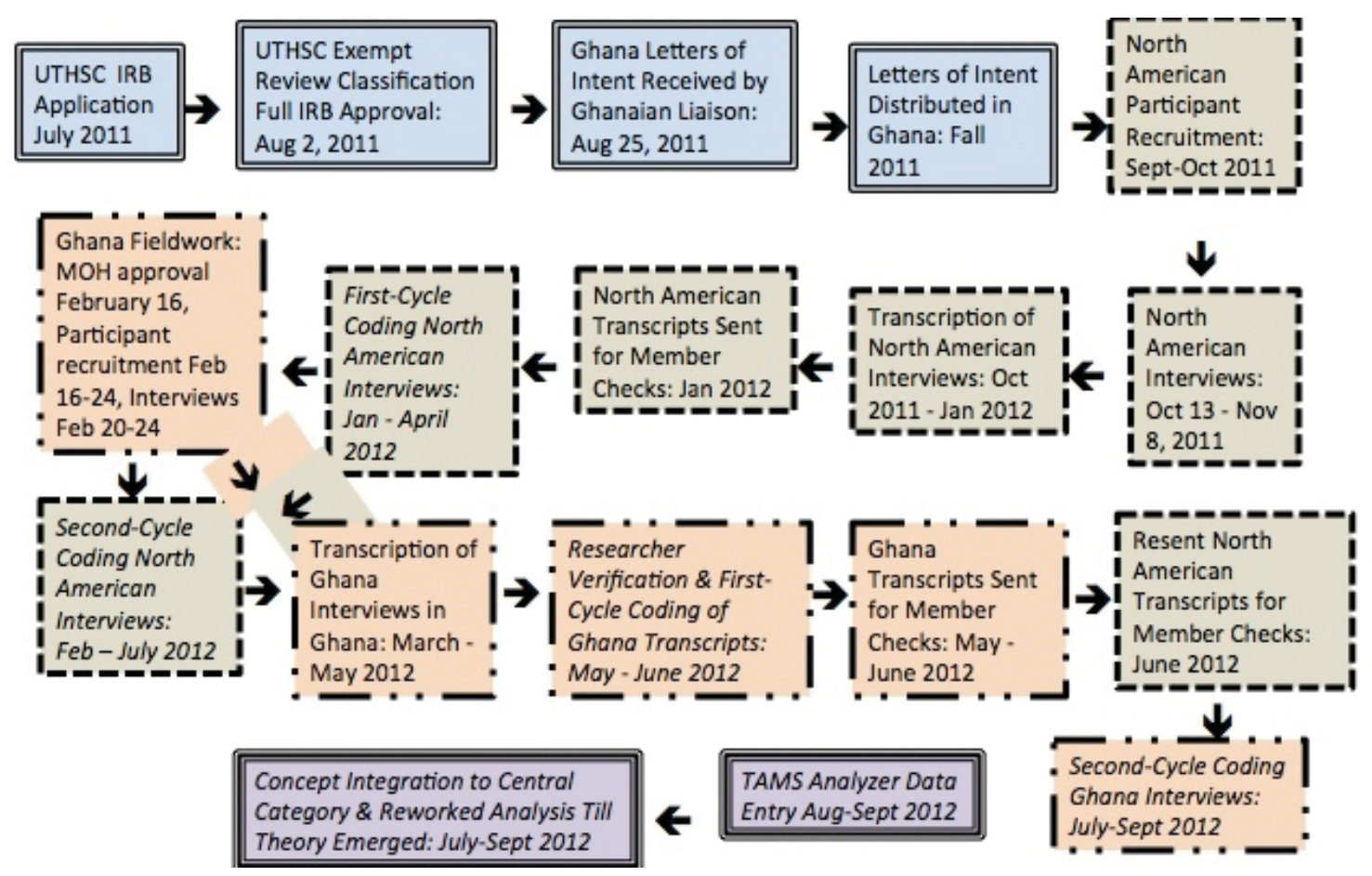

Figure 3-1. Timeline depicting study procedures.

Note: Light blue with double-edged border = human subject protection study procedures; tan with dashed border = procedures relating to North American data; peach with dashand-dot border $=$ procedures relating to Ghanaian data; light purple with italics $=$ study analysis procedures. 
knowledge base to function effectively and efficiently for their nursing education role in Ghana. Theoretical sampling continued, broadening the dimensional properties of concepts and adding variability to conceptual understandings by interviewing individuals from Ghana who were experienced in nursing education partnerships with outsiders. Interviews were conducted via phone or face-to-face.

Data management plans included an audit trail and constant comparison of data in an on-going analytic process that began with the collection of the first interview data. Various analytic thinking strategies served as tools for this researcher to recognize relationships between concepts, conceptually order and structure the phenomenon's dimensional properties, integrate concepts around a central overarching category, andrework the analysis until a logical, explanatory theory emerged to facilitate understanding of the phenomenon. The theory that emerged from this study has been grounded in the experiences and tacit knowledge of individuals with first-hand understanding of outsiders partnering for nursing education in unfamiliar settings. Insider and outsider perspectives afforded the opportunity to capture insights from both sides of a partnership endeavor regarding key information and knowledge needs to facilitate understanding of outsider role expectations in an unfamiliar setting. As such, the theory may be used to guide individual nurse educators through the process of acquiring information foundational to enacting their nurse educator role in an unfamiliar setting. 


\section{CHAPTER 4. RESULTS}

The purpose of this research was to generate a grounded theory to guide aspiring nursing faculty toward developing the necessary knowledge base to fulfill the nurse educator role in an unfamiliar setting. Specifically, this study sought to reveal insider perspectives and make relational tacit knowledge explicit concerning faculty role preparation processes for nurse educators going into an unfamiliar African setting. Study data arose from interviews with 16 individuals who possessed first-hand knowledge of partnering for nursing education endeavors in Ghana, Africa. Interviews occurred between October 2011 and February 2012. Purposive sampling provided an emic and etic perspective to the data, with 10 US outsider participants who had traveled to Ghana for nursing education endeavors, and 6 participants from inside Ghana who had worked with outsiders coming into their country for nursing education endeavors. Telephone interviews with US participants were completed with data saturation of the outsider perspective determined before face-to-face Ghanaian interviews took place. In addition to adding the insider perspective, Ghanaian interviews provided an opportunity to clarify outsider uncertainties that had been revealed during US interviews.

Key findings are presented in this chapter, beginning with a description of sample demographics. Next, the central overarching category grounded in this study's data is presented, followed by five main categories that help logically organize the processes participants experienced. After a brief overview of the five main categories, findings from each category are presented, including sub-categories and themes that helped reveal the properties and dimensions of each category. Properties for each category (Saldana, 2009) represented characteristics of each process step, and demonstrated the manner in which outsiders prepared during each process step. Dimensional aspects of each category (Saldana, 2009) are represented by sub-categorical and thematic variations that demonstrate participant's knowledge level along a spectrum toward the necessary knowledge base, and sometimes illustrate positive and negative connotations to that portion of the preparation processes. Exemplars are provided to enrich researcher interpretations, using participants' words to illustrate theoretical concepts at the subcategorical and thematic level. Finally, the chapter ends with a brief discussion and graphic depiction of the theory that intuitively emerged from the data, and logically links all concepts that were revealed through exploration of participants' tacit knowledge.

\section{Sample Demographics}

Participant demographics were collected at the beginning of each recorded interview, in response to questions 1a - 1e on the Broad Interview Guide (Appendix I). Those questions captured the (1) participants' role (Ghanaian host or US visitor), (2) timeframe and duration of the partnership experience, (3) institutional and organizational affiliations, (4) professional roles during partnership, and (5) prior or additional experience as an outside nurse educator in an unfamiliar setting. Descriptive demographics are presented in Table 4-1. 
Table 4-1. Sample demographics, Ghanaian and US participants $(n=16)$.

\begin{tabular}{|c|c|c|}
\hline \multirow[t]{2}{*}{ Demographic Characteristic } & \multicolumn{2}{|c|}{ Participants } \\
\hline & Ghana $(n=6)$ & US $(n=10)$ \\
\hline \multicolumn{3}{|l|}{ Timeframe for Experience (range: 1994 - 2012) } \\
\hline \multicolumn{3}{|l|}{ Status } \\
\hline Current and ongoing & 5 & $5^{\mathrm{a}}$ \\
\hline Completed & $1^{\mathrm{a}}$ & $2^{\mathrm{a}}$ \\
\hline Stalled, without forward progress & $1^{\mathrm{a}}$ & 5 \\
\hline \multicolumn{3}{|l|}{ Number of visits to unfamiliar setting } \\
\hline $1 \mathrm{x}$ & - & 3 \\
\hline $2-5$ & - & 3 \\
\hline $6-10$ & - & 1 \\
\hline$>10$ & - & 2 \\
\hline Missing data & - & 1 \\
\hline \multicolumn{3}{|l|}{ Duration of outsider visit } \\
\hline 5 days -2 weeks & - & 8 \\
\hline 15 days -4 weeks & - & 1 \\
\hline Missing data & - & 1 \\
\hline \multicolumn{3}{|l|}{ Number of years experience with outsiders } \\
\hline $1-4$ & 1 & - \\
\hline $5-8$ & 2 & - \\
\hline $9-12$ & 2 & - \\
\hline$>12$ & 1 & - \\
\hline \multicolumn{3}{|l|}{ Entities Involved in Partnership } \\
\hline Global health organizations/ initiatives & $6^{\mathrm{b}}$ & $3^{\mathrm{b}}$ \\
\hline Non-governmental organizations & $4^{b}$ & $5^{b}$ \\
\hline Institutions of higher education & $5^{\mathrm{b}}$ & $7^{\mathrm{b}}$ \\
\hline Professional nursing association & $1^{\mathrm{b}}$ & $1^{\mathrm{b}}$ \\
\hline Governmental agencies & $6^{\mathrm{b}}$ & $4^{\mathrm{b}}$ \\
\hline Healthcare institutions/ hospitals & $3^{b}$ & $3^{b}$ \\
\hline \multicolumn{3}{|l|}{ Professional Role } \\
\hline Visiting faculty to Ghana institution & - & $4^{\mathrm{c}}$ \\
\hline Host faculty & $3^{\mathrm{c}}$ & - \\
\hline Faculty abroad with outsider students & - & $4^{\mathrm{c}}$ \\
\hline Workshop/ continuing education instructor & - & $3^{\mathrm{c}}$ \\
\hline Administration & $6^{\mathrm{c}}$ & $3^{c}$ \\
\hline Regulatory oversight & $3^{\mathrm{c}}$ & - \\
\hline Consultant & - & $3^{\mathrm{c}}$ \\
\hline \multicolumn{3}{|l|}{ Other Experience as Outsider in Unfamiliar Setting } \\
\hline None & 2 & 3 \\
\hline $1-3$ countries & 2 & 4 \\
\hline 4 or more countries & 2 & 3 \\
\hline Experience in sub-Saharan Africa (not Ghana) & 2 & 2 \\
\hline
\end{tabular}

Note: Participant cited multiple status points ${ }^{a}$ multiple entities ${ }^{b}$ or multiple roles. ${ }^{c}$ 


\section{Theoretical Codes: Categories}

The central category that explains all aspects of the processes participants described during interviews for this study was 'Preparing for an Unfamiliar Setting'. Although the purpose statement and specific aims for this research targeted the exposure of role preparation processes, the data revealed that there were other preparation processes at a more basic level that effectively served as a prerequisite or co-requisite to role preparation processes. Preparation processes having to do with travel and safety considerations prompted me to realize that the overarching category to define all aspects of the study's data was in fact focused on 'Preparing for an Unfamiliar Setting'.

Researcher reflections while shifting and sorting data pieces during mapping of theoretical codes facilitated intuitive thought processes that led to the final recognition of five main categories that logically organize all of the data. Under the overarching category of 'Preparing for an Unfamiliar Setting' data were organized under five process oriented categories: 'Personalizing Information Needs'; 'Seeking Information'; 'Laying the Groundwork'; 'Developing Understanding'; and, 'Framing the Outsider Role'. Methodological notes helped track researcher recognition of process considerations that were revealed through the data.

Process considerations included the realization that although data demonstrated that 'Preparing for an Unfamiliar Setting' has sequential steps, the manner in which individual participants experienced the process is not linear, but fluid, as individuals interpreted their own understandings of steps in the process and recognized knowledge deficits along the way. Process varied for participants dependent on past experience, existing network and existing knowledge base, with each of those aspects serving as antecedents to each step of the process. Recognition of a deficit at any point along the continuum of 'Preparing for a Unfamiliar Setting' prompted re-exploration of earlier processes to expand outsiders existing knowledge. Lastly, interviews revealed that there is a negative side to many pieces of the process: instances in which the outsiders' actions, inactions, or responses could inadvertently create a barrier for working effectively in an unfamiliar setting. Categorical findings, including corresponding process considerations and exemplars to illustrate researcher interpretations are presented next.

\section{Category 1: Personalizing Information Needs}

US participants indicated their individual starting points, or perceived baseline knowledge at the initiation of the preparation processes by personalizing their information needs. The way participants interpreted their own baseline knowledge, and subsequent information needs, helped determine the manner in which they sought information. Data analysis revealed two main themes for personalization of information needs: (1) breaking new ground, and (2) drawing on experience. 


\section{Breaking New Ground}

Several US participants indicated they had not known where to start when they began to think about preparing for a nursing education endeavor in Ghana. Common themes included not knowing what was needed, what was available, who to talk to, what the context was like, and what the role would require in that setting. Instances in which participants lacked knowledge, or expressed uncertainty regarding how or where to acquire that information were grouped thematically under the sub-category 'Breaking new ground.'

"I personally felt like I was kind of fishing in the dark, I mean, I didn't know what I didn't know."

"I was pretty clueless that first time.... I had traveled extensively ... but I've never done that in that role.... I kind of went in blind to be honest with you, I had never taught before, I had never provided abroad before."

"I was the first nurse, and the nurses hadn't been invited to their previous [specialized healthcare workshops] ... they were trying to get the nursing profession involved in it."

"We didn't know any other faculty that had gone at that time, that had been there, or had done what we were doing, or planning to do... I wish we could have talked with other faculty that had gone there, or known who to contact."

"It made us realize that we need, exactly what you're doing, there needs to be some kind of toolkit or some type of guideline."

"Sometimes you don't know what you need ... you can have an idea of what it is that you think that you want."

\section{Drawing on Experience}

US participants indicated they drew from personal and professional experience, including the experience of other professionals, as they began to equip themselves for role translation to an unfamiliar setting. Demographics from this study's sample revealed US participant experiences as an outsider in Ghana ranged from those who had completed a one-time visit, to those with an ongoing partnership spanning more than 25 years, and corresponding visits too numerous to itemize. Professional associations, institutional and organizational affiliations, and existing networks served as demographical characteristics from which participants derived experiential knowledge.

"What I used ... was based really on my upbringing.... We traveled ... we always camped ... you kind of learn to go with the flow ... so it really helped me as far as going to a third world country... I was raised Christian ... respecting others and 
others' beliefs was also a big part of my upbringing, so coming and trying to force your ideas on somebody else is something I would never do - you just really have to learn to respect and adapt to whatever situation is in front of you."

"The first time we went over I went with a student, and I was fortunate in that she had former military experience and knew how to set up a mobile clinic."

"...Initially we probably used people who had past experiences as a guide, and [the clinical resource guides] they have developed."

"I've been in [specialization area] nursing for over 40 years, so I have a lot of experience and a lot of connections, both national and international."

"I do this a lot... I know what to look for."

\section{Category 2: Seeking Information}

The category, 'Seeking Information', refers to US participants' initial queries, in consideration to individually perceived levels of expertise, experience, and baseline knowledge. Regardless of how they personalized their information needs, all of the US participants sought information through purposeful networking and active literature searches. Data for 'Seeking Information' were organized under two sub-categorical headings: (1) networking, and (2) searching for information. Ghanaian participants verified that outsiders frequently sought information from Ghanaian insiders. The exemplars from the category 'Personalizing Information Needs' illustrated the fluid nature and ongoing learning that characterized the processes outsiders have undergone while preparing for role translation to an unfamiliar setting, as baseline knowledge levels were expanded to reveal newly recognized knowledge deficits and corresponding information needs.

\section{Networking}

Networking served as an avenue to make connections with individuals or organizations possessing some level of expertise or past experience with nursing education endeavors in Ghana. Networking as part of the preparation processes involved identifying potential contacts, then actively seeking encounters through professional or personal channels. After identifying those individuals, and making a connection with them, US participants solicited experiences, insights, and information from them.

"I talked to people... I talked to you, and I talked to another lady that is in XXXX, that had been over there and helped a school... kind of talked to her."

"I talked to other providers who had gone abroad before." 
"I utilized [name of global health program]'s baseline work, their goals and what they've found, why they're doing this ... that helped me."

"We met with a couple who had been in Ghana years ago ... and they told us a little bit of their stories."

"I work in a multinational city ... and many of my nurses are from Ghana, so I took the opportunity to talk with some of them who were also from Accra .... [I talked with a] nurse colleague who had gone on a previous trip."

"We have a guy that works at our college that's from Ghana... I ... talk[ed] to him.”

"The first time that I went there we had a contact in country who set up meetings for us, with ... faculty at a number of schools... other developmental NGOs [nongovernmental organizations] and government organizations, we met with people at the Ghana Health Service, we met with people at the Ministry of Health.... So that was just basically a meet and greet approach to find out what people were doing, to let them know what we were thinking, and to get people's ideas and buyins."

\section{Searching for Information}

All US participants described searching for information as part of the process they individually went through while preparing for role translation to Ghana. Participants explored online, hard copy, and library resources, nursing literature and related fields, and in-country (Ghanaian) publications. Searching strategies were characterized as general Internet searches, focused Internet searches, and reading from a broad array of publications.

"I ... went online to try to see if I could find guidelines."

"Broad Internet searches ... because it was really hard to find specific information, particularly for that country."

"We had students who helped us, students in our research courses... and they did pretty extensive lit reviews, general Internet searches, and using databases... CINAHL, PubMed, our library resources."

"Prepare as much as you can, read as much as you can... even if it's not geared specifically to faculty."

"If there isn't a lot of research on Ghana in the field that you're looking at, then go to related fields, to people who have done other things, to see their experiences." 


\section{Category 3: Laying the Groundwork}

Findings included preparation processes that laid the groundwork for nursing education partnerships in Ghana, and served to expand processes initiated while seeking information. The category of 'Laying the Groundwork' organized sub-categorical data that captured US participant experiences and recommendations to help guide outsiders through the processes of a) identifying key information topics that are relevant to the unknown setting; b) identifying key sources for retrieving information needed for the unknown setting; and, c) building relationships to facilitate access and effectiveness in the unknown setting. Ghanaian participants described what they had seen outsiders doing, and made recommendations to guide outsiders through the three sub-categorical processes included while 'Laying the Groundwork' for an endeavor in Ghana.

\section{Identifying Information or Knowledge Needs}

All participants identified information or knowledge needs as part of the preparation processes they had undergone as outsiders going into an unfamiliar setting for nursing education endeavors. Coupled with a participant's existing knowledge base, processes while seeking information (knowledge gained through literature reviews and knowledge shared through networking) helped US participants pinpoint the topics and areas of information that needed further exploration. Participants were asked to describe how they prepared for their role in Ghana; and also asked to identify topics for others to focus on when preparing for a similar nursing education endeavor A variety of topics emerged as key information needs for outsiders when going into an unfamiliar setting for nursing education endeavors. Analysis revealed 111 pieces of data that were coded under the category identifying information needs. Those pieces of data were grouped thematically as a) country level information needs, b) contextual information needs, c) travel and safety information needs, and d) cultural information needs. Exemplars are provided to illustrate each theme.

Country level information needs. Participants talked about searching for information about the country to which they were going. Findings revealed various dimensions to country level information needs as characterized by outsiders. One participant summarized the need to gain a broad country-level perspective. “... Going into any country, you need to learn about them, have a general view, not just the culture, ... because especially with nursing ... you are going to meet all groups of people [the diversity] ... the person coming in should have a fair idea ... of the big picture, and then narrow it to the ... situation."

Other participants shared specific dimensions of country-level information needs on which they had focused, and recommended types of information for other outsiders embarking on similar endeavors.

"I researched the country, the demographics, the British origin." 
"Trying to see... the things that they are battling... even when you're teaching in the curriculum, it may be different, because their country, and where they are, kind of dictates their health."

"Language, they do speak English, but it's kind of a British English...sometimes I actually even had trouble understanding what was said, or understanding the nuances."

"The burden of disease, what parts of the country are most affected."

"The number of nurses ... in the country, their distribution ... the number of health institutions, how ... they really fit, ... and even the organogram of the Ministry [of Health]."

"The Ghana Health System and the insurance scheme."

“Their scope of practice for nurses ... so you don't set them up [teaching toward what their scope allows them to do] Right."

"An understanding that their formularies cover basic classification."

"Other NGOs ... what [was] done, how far they've got to ... what are the gaps, and what was learned."

"The type of health facilities that we have, what the basic equipment in those facilities are, because at times you come in with your special skill, and your skill - to be frank with you - could be completely irrelevant, if you don't have the basic tools to work with, so if you want to go to a facility, match your skill to the type of facility you are going to."

Contextual information needs. US participants talked about the need for outsiders to learn about the context and day-to-day realities in the unfamiliar setting.

"What they needed to be prepared for in terms of just the environmental issues such as heat ... water, sanitation. "

"Sometimes electricity is there and sometimes it's not... Internet is slow.... Water, having to go and get water ... the [water] tanks, and all the inconveniences.... If you're going there, expect to be inconvenienced."

"You... really need to know and become aware of the lack of resources."

"In terms of the hierarchy of nursing ... like the head nurse, or charge nurse, the matron, I was always confused, ... Which one does what? That was just nomenclature, a lexicon I wasn't familiar with....The hierarchy in academics." 
"What pharmacists are allowed to do, because pharmacists actually have prescriber rights there and are able to treat."

"Health issues ... that are different from what you would see from wherever you are from."

Ghanaian participants talked about information needs for outsiders that were related to the setting's context.

"It's important for them to know about malaria and the signs and symptoms."

"The curriculum ... you take the cultural settings as a base, so for a course ... there were some aspects of nursing issues in Ghana."

"Those coming in from other nations, [want to know about] the educational structure.

"[Nursing] practice itself ... the individuals, the way they go about nursing."

"We have different levels... the certificate level, and now it's the diploma level, and the degree level... if you want to come ... What do they know? What level are we going to start our nursing knowledge from? Their level of thinking and so forth."

"If you want to go and study others ... What level have those people [obtained]?, What are the special educational frames for nursing? For teaching? It's important."

" The people, how they think and how they do their things [learner background and characteristics] ... I'm convinced that proper education is built on what the people have, and then you restructure it in such a way that it fits their thinking."

"They want to find out more about the institution where we are working ... [the institution's] values, the objective of your institution ... all the things that are expected of [the nurse educator] by the institution."

Travel and safety information needs. Participants identified a need to know about travel and safety considerations as part of outsider preparation processes. Ghanaian participants offered specific health and safety recommendations for outsiders.

"When people are coming ... it's important for them to protect their health."

"Consider preventative care while you are packing, and then be on the alert, tablets [malaria prophylaxis] while you are here, all of that." 
"Don't drink from the tap, so the water, the kinds of Ghanaian water, even bottled water ... there are specific ones [that are bottled correctly and safe to drink]."

A US participant revealed how her group had included travel and safety considerations in their preparation processes.

"We got all of our recommended immunizations... we did a lot of reading on communicable diseases indigenous to that area, we did our spraying of our clothes [malaria prophylaxis] ... we got trip insurance, made sure we had trip insurance so we could fly back when we needed to."

Cultural information needs. Twelve of the sixteen participants identified cultural information as a specific area for outsiders to focus on when laying the groundwork for work in an unfamiliar setting. Cultural information needs that were revealed through the data included awareness to cultural values and beliefs, accepted behaviors and norms, and existing sociocultural structures and hierarchies.

Ghanaian participants lent insight to cultural considerations for outsiders.

"You have to know nursing is a blend of science and art, and the cultural aspect of it is very important. When you're coming into this part of the world it is important to know about the culture."

"I think basically ... cultural differences ... it's not that Americans are straightforward ... it's just a cultural difference, how they relate, and it can affect their role in our context ... over in your country you can use everybody's first name easily without any offense, it's not ... in the Ghanaian society to be able to call someone who may be older than you [by their first name]."

"The relationships between lecturer and students... students here accord respect to their lecturers ... there's that kind of superiority ... a hierarchy."

US participants recommended specific cultural information needs.

"Find out about climate, behavior, cultural protocol, their understanding of time, taboos, and precautions."

"Family structures ... what makes women who they are, and what they are, the dynamics playing out there.”

"How decisions are made, particularly in the tribal community." 


\section{Identifying Key Information Resources}

All participants included identifying key information resources as part of the preparation processes for outsiders going into an unfamiliar setting for nursing education endeavors. Coupled with processes while seeking information and identifying what types of information were needed, the identification of key information resources helped participants to recognize where to go to access the information they needed. Participants were asked to identify resources they had used, and to recommend resources for others' use when preparing for a similar nursing education endeavor. Analysis revealed 124 pieces of data that were coded under the category identifying key information resources. Information resources that were repeatedly identified by participants and those that were highly recommended by participants as a key resource were grouped thematically. Those pieces of data were grouped as a) core nursing knowledge, b) key documents and publications, c) key informants and gatekeepers, and d) key organizations and institutions. Exemplars have been provided to illustrate each theme.

Core nursing knowledge. The theme of core nursing knowledge that emerged from the data supports this study's assumption of shared nursing knowledge among nurse educators, and relational tacit knowledge as a resource from which to share experiential knowledge. Participants relied on core nursing knowledge as the basis for preparatory learning to support a nursing education endeavor in an unfamiliar setting, for themselves, co-workers, and students traveling with them in an educational exchange capacity.

US participants shared how they had drawn from core nursing knowledge as an information resource.

"We use their nursing knowledge ... and we go over ... seven characteristics of the symptom, the diagnostic reasoning process, each ... of them are assigned a disease, tropical medicine disease ... they ... do the subjectivel objective assessment plan for that and we copy it ... put it into a big notebook, and each person has a notebook going in."

"I used, just the knowledge gained from my private practice."

"To prepare for that first [trip] ... I had a great Bachelor's prepared as well as Master's prepared education, and I just took that and modeled ... myself for the students."

A Ghanaian participant shared her perspective regarding commonalities among nurses, and their shared understandings.

"One of my counterparts said: 'nurses all over are the same' ... I mean it's even like in the house, if children would like to play a game, one will play a game with the other if you give them the opportunity." 
Key documents and publications. Participants specified resources they had considered key sources for information relating to partnering for healthcare in an unfamiliar setting. Key documents included global policies and guidelines for development efforts, and country level policies, procedures, and implementation plans.

Ghanaian participants highlighted global development guidelines and national publications as key information resources for outsiders.

"The Paris Declaration ... agreed [on guidelines for]... partners ... coming to developing countries to help."

"We have the National Health Policy, and we have the Five-year Program of Work, 2011-2015.... We also drew some information from [the] Ghana Poverty Strategic Plan.... So these are the key policies."

"We have the curriculum, we have a code of conduct for the nurses, a separate code of conduct for the midwives, we have clinical schedule books, we have log books for tutors, and then we have log books for students."

US participants pinpointed WHO publications as a key resource for information.

"The World Health Organization has an excellent resource, that puts all the medicines out there that each country has ... [their Essential Medicines Document?] Correct."

"The patient safety document [WHO] is very good, we use that."

Participants identified books as another key publication sources for outsiders' use. US participants identified books that had been written from the African perspective, and books that could be useful for helping outsiders understand the Ghanaian context.

"One theorist ... is Collins Airhihenbuwa, he's a Nigerian health behavior theorist ... and he has many publications, and a couple of books, 'Beyond the Western Paradigm' and 'Healing Our Differences'."

"On my way out of the airport in Ghana ... I found ... 'The Handbook for Community Pharmacists on the Diseases of Common Occurrence in the Tropics' ... and how to manage [the diseases].... If I went back I would certainly take this book, and ... read it beforehand."

"When I left Ghana I ... bought a little dictionary, you couldn't find it here in the States, but it was English to Fante."

A Ghanaian participant drew attention to books published in Ghana as an information resource for outsiders. 
"Many sociology books ... are written with the Ghanaian content.... One of our visitors who came, we bought a book from the University of Ghana for her, it was ... medical sociology and the view in Ghana.... It covers almost everything, the curriculum base, and everything, for students at the University of Ghana."

Several US participants recommended a NGO publication that is geared specifically to healthcare outreach efforts in low resource settings.

“The Hesperian Foundation's 'Where There is No Doctor, ... that's a good resource."

Key informants and gatekeepers. Participants talked about the people they spoke with to help them prepare for the partnership endeavor in Ghana. Informants included individuals from the US and Ghana with first-hand experience and knowledge about the unfamiliar setting. Ghanaian contacts often served not only as key informants, but also as gatekeepers for accessing information and other key contacts and resources in Ghana.

US participants talked about who had helped provide them information.

"I had a nurse midwife contact who was very key in helping locate the things that I needed ... who the right people were to meet ... to talk with."

"I work closely with the XXXX Health Minister, she's actually the medical director of our program, we felt it was really important to have oversight from a Ghanaian.... She is very much ... a gatekeeper/ consultant, she's very understanding. "

"People from the college ... would try to get me in with people to talk to ... about what was needed."

"People ... who were natives of Ghana who they could talk to, that would be helpful, healthcare workers ... nurses who work here [US] who are Ghanaian nurses."

"We had physicians and nurses who were planning the program from Accra."

"We ... used people who had past experience as a guide."

Ghanaian participants identified key informants for outsiders.

" $H R$ [Human Resources, $\mathrm{MOH}$ ], because a lot of things are done by $H R$... and ... the CNOs' office ... depending on what information is required, [the Chief Nursing Officers' Office, $\mathrm{MOH}]$ can quickly ... link up here and there" [link outsiders to whomever they need to meet]. 
"If you're going over to work with a particular school, ... they would send you ... materials, as part of your preparation, if they have it in print."

Key organizations and institutions. National, international, and global health organizations were frequently used as information resources. Several US participants had used the $\mathrm{WHO}$ and, or, CDC website as information resources during their preparation processes.

"I always go to the WHO website of course, and I always go to the CDC website, those are the two I start with."

"I... always recommend the CDC and WHO site."

"I remember using the World Health Organization, we definitely did that around the immunization issues that the students needed to be prepared for."

"I did look at the World Health Organization, basic health indicators."

Beyond the WHO and CDC, while searching health related organizations, US participants "... went to the ICN..."; and searched online to find "...experiences of different Peace Corp workers."

Governmental organizations, Ghanaian professional associations, faith-based institutions, and NGOs working in Ghana also served as information resources for outsiders. US participants identified governmental and NGO information resources inside Ghana.

"You can go to the Ministry of Health in a country, and often you can find documents on their website of - what their strategic plan is, what their priorities for the country are ... who the key people are you should be targeting that you want to talk with."

"We rely heavily on ... a church over there.... A lot of the young people at the church are really our cultural brokers."

A Ghanaian participant identified key governmental organizations as information resources for outsiders.

"The Nurses and Midwives Council ... have [a website]. The GRNA [Ghana Registered Nurses Association] also has a website ... the Pharmacy Council and GMA [Ghana Medical Association], they also have websites... GHS [Ghana Health Service] has its website."

Institutional programs served as another resource for outsiders while preparing for the unfamiliar setting. US participants described how they used their university as an information resource. 
"XXXX has an International Health Clinic, and so I tapped into that it terms of knowing what I needed to do physically to prepare myself in terms of immunizations and those kinds of things prior to getting my visa."

"Our 'programs abroad' here does a little spiel on culture shock, and we watch a video."

A Ghanaian participant identified institutional orientation in Ghana as an information resource to help outsiders prepare for role expectations.

"When they are in orientation [after arriving in Ghana] ... a three day orientation was done ... funding, and all the things that are expected ... by the institution.... [then] I took about a week to do orientation ... as far as the department was concerned ... small bites at a time ... orienting her through our lecture hall ... the skills lab demonstration room, then what ... we had ... and finally we looked at the curriculum itself."

\section{Building Relationships}

Analytic thought processes revealed common themes that indicated relationship building as a part of the preparation processes included while laying the groundwork for nursing education endeavors in an unfamiliar setting. Analysis revealed 94 pieces of data that were coded under the category building relationships. Concepts were grouped thematically as a) communicating, b) building trust, and c) envisioning future. Exemplars have been provided to illustrate each theme.

Communicating. Study participants indicated that advance communications and ongoing efforts to maintain open communications between individuals involved in an international nursing education endeavor were key elements to the success of such an endeavor. Findings suggested a tiered effect to communications, beginning with initial communications across continents, when introductions were made, preliminary planning occurred, and individuals began to become familiar with one another, in advance of outsider travel to Ghana. For the purposes of this study, higher tiers of communication, those occurring after outsiders made their initial visit to Ghana, have been grouped under the category 'Developing Understanding', and the theme of 'sharing'; or later, under the category of 'Framing the Outsider Role', and the themes 'listening' and 'working together'.

Participants with established networks (including key informants and gatekeepers) and relationships that had already been established were able to progress more rapidly to higher levels of communication. Dimensional aspects to communication and other preparation processes were evidenced by one US participant who had been 'Drawing on Experience' when 'Personalizing Information Needs.' When she spoke about those to whom she had talked while preparing for the unfamiliar setting, it was apparent she had strong antecedent characteristics (baseline knowledge, experience, existing network and 
existing relationships) and suggested those antecedent characteristics placed her at the level of 'Developing Understanding' very quickly in the overall preparation process.

"We met with a number of people that were ... working on midwifery education in Ghana, like JHPIEGO and IPASS ... other developmental NGOs and governmental organizations. We met with people at the Ghana Health Service. We met with people at the Ministry of Health."

Participant comments regarding communication technology capabilities in Ghana demonstrated the variability in communication technology capabilities in different parts of Ghana; and served as a reminder of change and growth in communication technology in unfamiliar settings. Preliminary communication needs, including verification of actual communication technology capabilities for partners' use were revealed through participant recommendations.

"[You should] have contact with the school that you're going to, as much contact as [possible], if they have the technology. I know with XXXX we ... Skyped back and forth; but, obviously we didn't have that technology with Ghana. [For Ghana] we were emailing, they had one computer. " [US participant speaking, first Ghana trip 2005, to an area a few hours from the capital city.]

"During the planning stage, they had come [Ghanaian participant speaking, active partnership underway in capital city], we planned together with them. We had a series of teleconferences and ... video conferences, Skype ... a number of them."

Preliminary planning and preparation processes included two-way communications where partners listened to one another and shared goals. A US participant noted:

"We did discuss what their objectives were, for us coming; and, we also shared what our objectives were."

US participants recommended purposeful attention to logistics while preparing for travel to the unknown setting. The theme of communicating included logistical planning, so members of the team would know how to proceed. Part of the logistical planning process was simply allocating enough time to adequately discuss, plan, and prepare for the intended nursing education endeavor.

"Set times aside. Meeting times where you can get together if you're going with a group of people, to just discuss and talk about your planned trip, what are your aims, what are your goals, what can you do best to prepare."

Advance preparations for some US faculty included logistical planning to communicate safety considerations to students and other members of the US team. 
"With a student population you have to ... think about drugs and alcohol, and you have to go over that with the students, and let them know what the expectations are, or about the consequences of those two issues in a foreign country." Another participant noted "That's an in-depth part of our preparation... we talk about ... water safety, not eating street food, and why... not being out at night, no alcohol consumption, lots of injury prevention, and endemic disease prevention.... Things are very different post 9-11 ... taking undergrads and grads, safety is very important."

Another aspect of communicating emerged from the data that involved challenges participants experienced when communication attempts proved unsuccessful. Common findings for US participants that had indicated they were 'Breaking new ground' when they began their preparation processes included difficulties 'Laying the Groundwork'. Ineffective communications, ineffective networking, and consequently inadequate access and failing to establish trust and build strong relationships were manifestations of difficulties 'Laying the Groundwork'. Outsiders experiencing those manifestations expressed uncertainty and their partnership efforts seemed stalled, without forward progress.

"It seems like everything is kind of based on, I don't know --- who you know, and if you can get in and talk to people ... so there were a lot of barriers ... actually figuring out exactly what was the process, and what was required in the process, and who things had to go through ... even to link up... with somebody else that has the knowledge ... and maybe got tidbits, that was even kind of closed.... If I could have found something ... online that could ... help me know the process ... that would have been helpful, but I don't think that exists."

Building trust. When discussing the processes they underwent while preparing for the nurse educator role in an unfamiliar setting, participants indicated that developing trust between partners in different locations was a major part of 'Laying the Groundwork' for an effective partnership. Participant experiences and reflections indicated that establishing trust can be a lengthy process, and that outsiders' are often not prepared for the idea that trust simply takes time to develop. Regarding the process of establishing trust, one US participant noted:

"I have colleagues that just think they can go in some place, and start doing stuff; and it's a very difficult concept. There are few of us out there, and I say this in a very thoughtful way, there are few of us out there that think about it from the vantage point that it's a process of ... both community based participatory practice and research to establish trust before you can just go in with students... people in universities are just wanting to take kids overseas, and you can 't, you can't do that in clinical work." stated:

Reinforcing the concept of taking time to establish trust, another US participant 
"You just don't find out on a first fact-finding trip. It takes time to establish those kinds of relationships with people, and I think that's the power of relationships."

A US participant revealed her perception that initially there seemed to be a suspicious attitude, when insiders have doubted outsider intentions.

"There's also sort of a mistrust.... Why are you wanting to come in? We'll take it, but are you going to stay long-term?"

Findings from US participants revealed that although trust takes time to develop fully between partners, transferred trust that comes through referred networking might be advantageous at the beginning of a partnership. "It's about reverence, and for the people that are going in, there needs to be somebody who has worked hard to lay some foundation, and complete reverence for the community of interest." Some outsider participants benefitted from their association with outside institutions or organizations that already had an established and trusted relationship with targeted Ghanaian partners. "When I would say to somebody that I was from the University of XXXX, they all knew somebody from there, so that was very helpful." Other outsider participants referenced personal connections that lent credibility to their efforts in Ghana: "A big part of that was the interaction between $X X X X$ and the pastor at this church." Another US participant noted: "I think XXXX's good reputation was a huge facilitator.... When he lived in Ghana he had built something like 24 churches and ... about 16 schools... they knew $X X X X$."

One US participant revealed that it had taken her years to establish complete trust and reap the full benefits of having strong in-country connections, with full access to information and other in-country resources.

"For me ... just having the trust and the connections has pretty much given me the access.... I would say that ... I've had complete trust and complete access for about two years now... [Researcher: If you began 11 years ago, that was still about 9 years toward building up the trust?] ... Absolutely."

Envisioning future. Another dimension related to 'Laying the Groundwork' was reflected in US participants' comments as they spoke about existing goals, long-term plans, and future possibilities with their partnership endeavors. Funding seemed to provide evidence of long-term commitment to the nursing education endeavor, and helped lay the groundwork by helping build trust between insider and outsider partners, and also by enabling future planning.

"Their trust in me ... really started with the first grant ... That we weren't going to disappear... we wrote it with them ... they were co-Is [co-investigators] on it."

Other aspects of envisioning the future included being open to new opportunities, and transitional planning for changes in organizational management of long-term partnership endeavors to facilitate sustainability. 
"I'm open to having ... international exchanges. I don't mind even sharing our faculty if we can, we're open to the future and what possibilities there may be."

"When I started [the partnership effort] I was at the University of XXXX, and then

I retired, and ... one of my former students who teaches at the University of XXXX ... for the last two years they have pretty much provided the students and practitioners [outsider participants going into Ghana] so we are in the process of transitioning the organization ... to the University of XXXX."

\section{Category 4: Developing Understanding}

Study findings included preparation processes during which participants attempted to develop an understanding of issues and considerations relevant to the unfamiliar setting they were preparing for. The category 'Developing Understanding' captures preparatory processes related to knowledge acquisition, as participants interpreted what they had learned and what they had questioned while 'Laying the Groundwork'. This category includes preparatory processes in advance of travel to Ghana, while participants gained a beginning understanding of the context from information resources while 'Laying the Groundwork'; and, also includes continued learning and role preparation processes acquired through experiential learning in the Ghana setting. In circumstances where an outsider had not already developed relationships and networks with insiders (who could serve as trusted information resources to aid outsiders' understanding) the processes revealed while 'Developing Understanding' prompted the need to build those relationships and networks for effective communications about the unfamiliar setting.

'Developing Understanding' categorical findings have been organized under subcategories to reveal participants' knowledge and information interpretative processes related to a) clarifying, b) defining, c) experiencing, d) figuring it out, e) internalizing, f) recognizing, g) sharing, h) not knowing, and i) questioning.

Data revealed various themes in each sub-category. An expansive review of each theme is beyond the scope of this dissertation. Exemplars are provided in participants' words to illustrate assorted themes under each sub-category.

\section{Clarifying}

Clarifying included participant experiences that were clarified spontaneously or expanded upon through probing questions during interviews. The sub-category of 'Clarifying' also captures researcher-solicited clarification from Ghanaian participants regarding issues identified through preliminary analysis of US participants' data, concerning issues outsiders had questioned or not understood about the unfamiliar setting. Themes were used to group dimensional aspects of the sub-category 'Clarifying' to illuminate how outsiders' developed understanding related to: accessing information, 
existing nursing regulations, organizational structure of healthcare services and nursing education, developing trust, and cultural considerations for the setting.

Outsiders, especially those who had been 'Breaking new ground,' had expressed confusion and ineffective communications while 'Laying the Groundwork.' Clarification was sought from Ghanaian participants regarding the processes or protocols necessary to gain access to information, open doors to existing networks, and begin to build relationships inside Ghana. Gaining a better understanding of the organizational structure for healthcare services in Ghana served as another dimension to the process of clarifying information for a foundational understanding of key informants and power brokers for healthcare in Ghana. Ghanaian participants clarified and explained protocols and organizational structures that outsiders need to understand.

"If you needed a document, you needed to write a simple application [submitted formally]... identifying yourself - I am XXX, and occupation, requesting the document, saying I need to have a copy of your [as an example] curriculum. Officially - and officially it will be given to you. The essence is that it contains classified information and wherever you want to reference or use it, you just acknowledge us."

"It is the Ministry of Health who represents the government, any other institution dealing with health falls under this Ministry, so you can not walk straight and talk partnership without involving the Ministry, it may get to the point where that institution would be limited for writing the kind of support or different kinds of recognition, and it would be too late for you to contact the Ministry."

"The Ministry ... has the agencies for implementation and the regulatory bodies to ensure compliance to standards.... They are all implementing agencies under the Ministry of Health. The Ministry is the head, formulating the policies that are sent to specific agencies for implementation, of which Ghana Health Service is one.... Under the Ghana Health Service we have regional services, district services, and then sub-district services, and then to the community level.... We have ... the GRNA [Ghana Registered Nurses Association] ... for labor issues, salaries, negotiations, and then welfare in general, and the PPME [Directorate inside MOH: Policy, Planning, Monitoring, \& Evaluation] office of quality, policy, direction, ... [and] implementation."

"Now nursing has come to the tertiary level, so the requirements to enter nursing are just like the requirements to enter university.... Tougher, tougher ... it's not going to be easy at all.... It's a way of shortlisting and then getting the best, it's just that there are too many applicants."

Participants clarified issues relating to regulations that guide nursing education and practice in Ghana. US participants clarified the expectation that outsiders should honor and respect existing regulations. 
"You have to respect their rules, their nursing practice acts ... understanding what liability would be involved if there was a legal issue, as far as malpractice."

Ghanaian participants clarified the organizational structure for regulatory oversight of the profession and where to find the information.

"We call it Nurses and Midwives Council of Ghana.... The law that established the council has a three pronged [purpose]. It says the council is mandated to regulate the education, the training and the practice of the profession.... The requirement for entering our licensing exam according to nurses is advertised on the Internet on our website."

A Ghanaian insider provided a glimpse of past events that have influenced insiders' perspectives when working with outsiders. The attitude of mistrust from insiders as perceived by outsiders, and the need for time to develop relational trust while 'Laying the Groundwork' can be better understood when consideration is given to historical context.

"Some, I'm choosing my words carefully ... the picture with some health partners is that instead of giving technical support to us, so that they will be behind us and then supporting us for implementations, they want to play a frontline role to implement ... which is not too good, because in the international arena, it's not permissible.... [for example] If XXXX comes and says 'I am a health partner, I have this bottle of water, but I'm interested in only three schools in the Southern Belt.' Three schools! We have 23 institutions here! ... What informed your choice?.... We are into regulations, with such orders for all the schools.... So that if you come and say 'I am interested in these three schools' you will end up putting the three schools ahead of the other 20."

Cultural protocols and cultural norms represented an area of uncertainty for outsiders. Ghanaian participants clarified understandings related to expected behaviors and cultural etiquette in the Ghana setting.

"It's ... a cultural difference, how they relate, and it can affect their role in our context.... Over in your country you can use everybody's first name easily without offense. It's not catching up in the Ghanaian society, to be able to call someone who may be older than you ... [Researcher: So the respect and honor for the elders?] ... Yes... [and] for superiors .... If you went into a class where maybe they could be older ... have not traveled, or have never met an outsider before, and you use their first name ... they may consider it rude... I remember one event where someone, a much, much younger person came to my office. She kept saying $X X X, X X X, X X X$, [calling first name] ... then my secretary, who was about her age or older, had to tell her that over here ... we don't call our seniors by their first name." 
Culturally appropriate modes of address were clarified as a mechanism to enhance understanding of expected communication patterns. A Ghanaian participant clarified that Madame was the proper address for women in Ghana, while the proper address for men was clarified as "Sir or Mister, if you know the name you can call him Mister XXXX."

The Honorable XXX was clarified as being reserved for Ministers in the government, and not appropriate for other high-ranking governmental posts. "The Registrar would not have [the title] The Honorable.... It's only [for] the Ministers."

\section{Defining}

The sub-category of 'Defining' captured large amounts of data coded to represent various themes that emerged as participants identified issues and concepts they felt were important for outsiders to understand about the unfamiliar setting. Specific question probes during interviews prompted the large amounts of data, as participants responded to requests to: identify the barriers and facilitators outside nurse educators encountered while developing the knowledge base to assume the nurse educator role in an unfamiliar setting; and, tell the researcher anything else they considered important for outsiders to know when preparing for role translation. Analytic thought processes prompted the realization that the sub-category 'defining' included participant responses that were organized according to themes that defined a) barriers, b) facilitators, c) existing efforts, d) needs and wants, and e) safety and travel considerations.

Barriers. Findings were coded to organize barriers participants defined. Analytic thought processes and researcher reflections prompted the realization that some of the barriers participants defined were a result of outsiders not understanding, not connecting, not knowing, or questioning something. Their lack of understanding, knowledge gap, or ineffective networking created the barrier in those circumstances, and represented instances where participants had not been successful in one or more of the preparation processes for 'Personalizing Information Needs', 'Seeking Information', 'Laying the Groundwork', or 'Developing Understanding'.

Barriers that arose as a result of US participants' lack of understanding, existing knowledge gap, or ineffective networking included issues related to the context such as cultural considerations, tribal beliefs, time interpretations, language, unfamiliar nomenclature, outsiders not following existing regulations, and difficulties interpreting the structure of healthcare in Ghana. US participants talked about barriers they had encountered.

"One of the biggest barriers that you see is that when newcomers come in they don't respect and value the culture."

"It's difficult because you don't speak the language, for the most part."

"They [Ghanaian institutions] don't have ... things on their website, like a faculty handbook.... You don't know where to go." 
"People just ... think they're going to go down there and just start practicing without respecting the government of the country."

"My idea of a timeline follows a timeline exactly, and their idea of a timeline is not quite that rigorous."

“Time was a [barrier].... They use this phrase a lot: 'If God wills' or 'By God's grace'."

A Ghanaian participant identified language barriers related to variations in terminology between the two countries.

[The] “...physician's assistant in the US ... in Ghana we call them medical assistant, so maybe in the US if you talk about physician's assistant, that says you have a bachelor's degree, that says you'll be able to do A- $B$ - $C$-whereas in Ghana our medical assistant would be a practicing nurse for 5 years, before going back to school to do post training beyond their program."

Other barriers arising from participants' lack of understanding included misinformation that participants may have received, inappropriate outsider attitudes, and unsustainable interventions. Ghanaian participants identified instances of barriers resulting from inaccurate outsider understandings.

"Another thing I observed was that when ... [there is a] transfer of some processes from second- hand knowledge, some are misinformed.... And sometimes it's difficult to correct them, especially there might be some misinformation, and the person might also encounter something that may concern somewhat far away, so when you try to say: This is one in a thousand, not a normal occurrence, it becomes difficult sometimes."

"We have different levels of development -- and of course - the truth also - is the fact that [when] one is not understanding one's point of view does not necessarily mean that that is not right -- many do that."

"I think one of the things is - a lot of the times when Expatriates do come, they come with the 'We know what's best' -- and I think that immediately throws up --throws up some walls."

US participants identified barriers that can arise when outsiders lack understanding of best practices for developmental aid.

"Ethical issues ... as far as taking medicines ... that aren't needed and putting people at more of a dependent role on Western medicine."

"I think band-aid medicine is a terrible thing to do, slap a couple of band-aids on it and then withdrawing and not leaving any healthcare." 
Barriers defined by participants that were not linked to ineffective outsider preparations prior to travel included issues related to reality considerations such as costs associated with partnering at a distance, time limitations for host and visitor partners, being recognized as an outsider, resource and environmental realities of the setting, and associated stressors while in-country. US participants shared barriers they experienced as a result of reality considerations.

"Lack of time to prepare. We kept saying we were going to do this and do that and get this ready ... time runs out. Before you know it we're leaving tomorrow!"

"One of the things that you really have a hard time depending on is Internet connection."

"I think ... one barrier was that I was foreign - and I was identifiable as foreign." "It was really hot, just environmentally ... it was 120 degrees.... We were really uncomfortable, which doesn't make for [a] good frame of mind when you're giving a lecture and you want to meet with the faculty."

"It's more about keeping spirits up ... it's stressful."

A Ghanaian drew attention to cost considerations as a barrier, with an emphasis on the brevity of many outsider visits in consideration to associated costs.

"Airfares - there is also the cost - to have someone come here and stay for two weeks and then go away."

Facilitators. The theme 'facilitators captured US participants' interpretations of what served as a facilitator for them while they attempted to acquire the knowledge base and information needed for role translation to the unfamiliar setting. Ghanaian participants defined what they perceived as facilitators for outsiders during a nursing education partnership endeavor in Ghana. Analytic thought processes and researcher reflections prompted the realization that some of the facilitators participants defined were enhanced through knowledge gained during preparatory processes while 'Personalizing Information Needs', Seeking Information, or 'Laying the Groundwork'.

Facilitators defined by participants that were a reflection of effective preparation processes while 'Seeking Information' and 'Laying the Groundwork' included networking and communications via gatekeepers or in-country liaisons, powerful advocates, and trusted associates. US participants identified human resources in Ghana as facilitators.

"When I got there, there was a nurse who had worked for the Nurses and Midwives Council.... She was invaluable to me in terms of getting information." "She had a, sort of big country picture.... Sometimes you go in and you meet with someone at a school and they know what's in their Region or in their school, but 
they don't really have the big picture. So that was really helpful to be able to have contacts with people that had the big picture."

Other facilitators defined by US participants involved outsider attitudes that readily adjusted to the setting, and behaviors that demonstrated honor and respect to the Ghanaian people.

"I think the matter of asking rather than telling."

"I think anybody, if you ask respectfully... will give you information that will be helpful to you."

"I found one of the things that was helpful was knowing some key words in the language."

"I found that that was one of my door openers, it was like the secret weapon.... I would always ask, what's your name? And they would say their name and I would say, get this in your head... if I saw them at the cafeteria I would say Hello so and so, and they would ... [exclaim] You remember my name! And that would get me into so many places!"

"To be able to adapt to things very easily ... I think that's really helped me."

"I would say ... being a good teacher, that is using a participatory style, which is something that they're very used to, using case study approach not lecture."

Existing efforts. Two participants defined learning about existing efforts in Ghana as a part of the preparatory processes recommended for outsiders. A US participant recommended that outsiders should "find out what people are doing." One of the Ghanaian participants defined why it was important for outsiders to learn about existing efforts. "Know what is there so that they can ... prepare ... we need preparation so that there is no duplication and frustration." The process of 'defining existing efforts' built on communication and networking preparation processes that may have been initiated while 'Seeking Information' and 'Laying the Groundwork. In instances when outsiders did not have existing networks or relationships to facilitate communications in country for accessing information about existing efforts, findings revealed the need for outsiders to develop those resources as part of 'Preparing for an Unfamiliar Setting'.

Needs and wants. Participants defined understanding the needs for the setting and the wants of insiders as an aspect of 'Preparing for an Unfamiliar Setting'. US participants defined the need for outsiders to understand what insiders wanted and needed, so interventions and efforts could be geared to address those wants and needs.

"I did a needs assessment based on what they wanted."

"The biggest thing I needed to do was to assess their level of knowledge. 
"They were wanting the Bachelor's degree, so ... if you're going to develop something it needs to be a Bachelor's degree.... And then they were talking about specialization of those nurses, that they wanted more like that.

A Ghanaian participant defined wanting sustainability for endeavors and interventions with outsiders.

"There is ... potential ... but I would want it to be sustainable."

One US participants defined insiders' needs, shared those needs with her successor, then described the successor's response, illustrating another dimension to the concept, when outsiders do not respond to insiders wants and needs.

"That was supposed to be part of the criteria when I gave feedback after that visit ... that they need hands on [demonstrations and return demonstrations] and the woman who followed up on me didn't do it. She said: No, no, no."

Several participants defined personal wants and needs while preparing. Personal wants and needs for US participants related to helping equip themselves and other outsiders for role translation to an unfamiliar setting. Two US participants talked about needing a mentor to guide them through the process. Participants' comments revealed challenges they had experienced while trying to identify a mentor.

"I need a mentor, that's the problem."

"I tried to get a mentor before I went that I knew there, somebody, I wanted to make a contact there."

One US participant validated the need for this research study as a mechanism to help equip other outsiders.

"We need to, like what you're doing, we need to pass this information on.... because it will build a body of knowledge on this."

Safety and travel considerations. Participants began to develop an understanding of issues related to safety and travel by defining what those considerations were and interpreting information and knowledge gained while 'Seeking Information' and Laying the Groundwork'. They added to their personal understanding of 'safety and travel considerations' after having first-hand experience in Ghana. Participants' definitions reflected their dimensional understandings of the concepts, as new information, knowledge, and experience were gained regarding safety and travel considerations.

Participants defined travel considerations related to the logistics for obtaining a visa and being allowed to enter the country. Outsiders can apply for two different types of visas when making application to enter Ghana. One visa will allow one visit to the 
country, while the other type of visa will last for 5 years. One US participant defined her visa as one that would last for "five years", thereby facilitating multiple visits to the setting. A Ghanaian participant who had worked with multiple outsiders coming into his country illustrated consequences of not understanding travel limitations imposed by limited visas. "Some of them have just an entry that may be for this much and they don't know, and they pop up from outside the country ... not knowing they don't have multiple times to come, they get stranded [at the airport, unable to get through customs and put back on a flight to the US]."

An insider highlighted travel considerations for outsiders when applying for a Visa to enter the country, and safety considerations to enable contact while in the country.

"It is not easy to get a Ghanaian visa, I'm told. Know where to get the visa, the application. [When applying, tell the Ghanaian embassy] that you have an invitation for a collaboration, and say that to them [the insider partners] so they can go to the Ghanaian consulate [on outsider's behalf], those at the university. And when they come [outsiders in to Ghana] you have to let their country know that they are here with you."

A Ghanaian participant defined safety considerations while inside Ghana that are related to dangers outsiders can inadvertently place themselves in when they have not followed Ghanaian policies and regulations, or have not understood that there were policies and regulations they should be following.

"People may have very good intentions and motives. But if it's wrongly done, in the first place you put yourself in danger. But when it happens nobody protects you. [If outsiders have not gone through proper channels and obtained the permissions required] You don't have the government or the authority to do that. We shouldn't assume that everything will be well. Of course, when everything goes well 100\%, praise God. But in case something happens, then who will protect you?"

A US faculty member who has taken nursing students to Ghana defined recent changes in her safety and travel preparation processes.

"This year I'm going to stress, just because of all the natural disasters going on, about the American Red Cross, and if something were to happen how they get on the American Red Cross site and click that they're OK.... I have not done that in the past but because of everything going on I just want them to know that if they somehow got separated that they need to know how to do that."

She also defined how she had adapted preparations for safety and travel considerations as a result of experiential learning across the years. 
"I... register with the State Department ... as a group, so everybody signs in and puts their address and their first contact.... At this point in my career I carry an emergency kit with me that I don't use with patients at all, only with my students. If there's an issue ... down to a band aid, all the way up ... I take sutures and stuff, just in case, and then I always take extra medicine... all that kind of stuff."

\section{Experiencing}

US Participants (7) credited 'Experiencing' things first-hand as one of the processes through which they developed an understanding of the unfamiliar setting. Experiential learning was revealed to be an ongoing process through which outsiders continued to acquire knowledge and increase personal understandings of contextual realities. Participants with multiple years of experience talked about ways they learned across time, and how their experiences during early visits to Ghana enhanced their preparations for later visits, demonstrating dimensional aspects for this sub-category.

"The more times you go the better at it you get. Every time I go, even right now with students, I learn something, every time, some new disease I was unfamiliar with, or some new medicine --- something."

"Knowing more about their cultural beliefs and so forth, that was something the first time, that we may have been uncomfortable with, that we were more comfortable with the next time."

"We provided care ... that [first] time, but the supplies that we had with us were rather sketchy, so that gave me some preparation, that the next time I went I knew how to structure it ... I also knew what we needed in order to provide primary healthcare to the community."

US participants acknowledged personal experience as a key factor in their ability to understand the setting, and recommended first hand experience as an essential element for others wishing to understand the setting.

"I think until you see it, no matter how much you read on the environment, the pictures, until you're there and you see it, you feel it, you smell it [you can't understand] because it's just so different."

Whether you're in the nurse educator role or provider role, you just have to go there and really experience it, and see."

"It takes ... being in the classroom, and being with the people, to figure out what kinds of resources they're using. How are they making decisions about ... who passes, who doesn't pass? How are they evaluating students? Are they evaluating students? How are they being evaluated?" 


\section{Figuring it Out}

The sub-category of 'Figuring it out' as a dimensional aspect of 'Developing Understanding' represented an extension of experiential learning, in which participants reflected, thought, and reasoned their way through first hand experiences to further understand the unfamiliar setting. The sub-category of 'Figuring it out' included thematic dimensional aspects whereby outsiders adapted, assessed, compared, interpreted, and translated their understanding of issues related to culture, education, the healthcare system, existing protocols, the unknown, and local ways of doing things in the unfamiliar setting. There were 97 pieces of data coded that were grouped under the sub-category 'Figuring it out'. Data codes were assigned under this sub-category for responses from all 16 of the participants. US participants described how they went about 'Figuring it Out'.

"I found a dilapidated board to do diagrams, and to explain that breast cancer can be local or systemic disease, that still wasn't understood, and yet they were talking already about these other treatments, but if the nurse didn't understand that [it could be local or systemic], it was all Greek to her. So...within 10 minutes I devised 10 questions to ask them, and I had brought little give away things, and they got to earn prizes ... if they knew the answers to these questions, which would give me feedback on how much they were absorbing.... so I ended up going back and re-teaching a lot of the things that were taught at the medical level, and brought it down to the nursing level."

"There are a lot of [mortality] causes that we would not even envision, like in the area I was working, the idea that if your child isn't born through the vagina it doesn't have a soul, and what do you do with a soul-less child? So women will wait for the very last moment to go to the hospital if they get in trouble, and because they're so anemic nutritious-wise, and malaria-wise, that a normal bleed for them may be a mortal bleed, so you have to recognize that you have to look at different signs and symptoms."

"When we have satellite clinics I always visit with the chiefs and the elders to ask their permission, and when I was doing some research into maternal mortality I would always visit with the chiefs and the elders to ask their permission to visit with the women."

"To me it was ... it's still a ...fractured [healthcare] system. Everything is in silos [sections of healthcare isolated from one another]. I especially found that out on my second trip, where I spent a week of my own vacation time, and all I did was walk around, and try to figure the system out."

"What does that mean? [a local phrase] - and I talked to somebody and they said: Well - By God's grace it won't rain, and wash out the road, and the car won't break down. So if they say 'By God's grace' what that's saying is: I will get there at this time if possible, but if not possible, God graced it to be later so don't get ... twisted about it." 
"You've got to come in and help them, with providing the best care and showing the ways that we would do things; but, also integrating it with the way they do things ... [balancing those perspectives to do] no harm."

A Ghanaian participant provided an example of how they had adapted the way the NMCG provides educational offerings to outside nurses seeking professional licensure in Ghana, thereby facilitating outsiders understanding of the process, and helping outsiders figure out the system.

"We have modified it a little. In times past we used to bring experts here and we would put all the foreign trainers here [to Accra, the capital city]. We have Indians, we have Americans, we have British. We would put everybody here and then we would bring experts. You know we are in the sub-region where malaria prevails. We go Ghana Malaria Control Project, and we bring the director to come and then they would come and do a presentation on updates of malaria. Or bring TB HIV/AIDS and all those things. Now we have modified it a little. We do it at the regional level for them."

\section{Internalizing}

The sub-category 'Internalizing' represents researcher interpretations of instances in which US participants' comments represented conscious efforts to not impede interactions in the unfamiliar setting through outsider attitudes, actions, or responses to cultural differences. Themes that emerged from the data that lent dimensional aspects to 'Internalizing' included acknowledging, adapting, being accountable, being open, being patient, having cultural humility, not complaining, respecting, reverencing, and valuing. All 10 of the US participants made comments that were coded under the sub-category of 'Internalizing', and interpreted by the researcher as a part of their preparatory processes while 'Developing Understanding' of the unfamiliar setting.

"One of the best interviews I ever had was with a traditional birth attendant that was certified.... I asked her how that certification changed her practice, and she said: 'Well it's really neat they gave me a pair of scissors instead of this rusty old knife I used to use to cut the cord, and I don't go out into the back and gather up these green herbs to put on the stump after I've severed it, and I found out that it really doesn't hasten labor when you beat a woman with a broomstick.' So you know, you have to leave yourself open to hearing all sorts of thing."

"There's a big difference between wanting to participate in international healthcare and clinical tourism ... I saw it a different way and so I ... spent a lot of time researching ... the humility piece and I've learned about the people as I've gone."

"If they have a cultural or a healing process that has no scientific or evidence based research or whatever behind it, but it's doing no harm, [leave it alone, 
respect their way] What's the problem?... Never coming in and trying to show them up - and saying the Western way is the best way."

"You ... have to learn to respect and adapt to whatever the situation is...."

"If we [outsiders] can just get to a place where we value somebody's perceptions of health, and not always be functioning in a sort of a silo of ethnocentrism."

One US participant shared an experience she had with another outsider that was a reflection of that outsider's not understanding and not internalizing the way her comments might be perceived by insiders.

"She was saying ... this going off of electricity ... she would complain to people about it, and I pulled her aside and I said: 'They can't change it! ... You're making them feel like you're looking down on their country'."

\section{Recognizing}

The sub-category of 'Recognizing' captured instances in which US participants talked about things they came to understand after seeing and experiencing the realities and actualities of the setting; and instances in which they demonstrated a recognition of limitations, and preconceptions relevant to the setting and partnership effort.

"When you're somewhere else you may have to deal with issues differently than when you're here [the US], ... and I knew that we would have to do things their way, it couldn't be my way."

"It's a pay as you go system, so ... a woman may come in and get a biopsy, but to get the biopsy read is a separate charge ... so patients literally go home with their specimen until they get the money together to get it read.... basic things that we utilize aren't in that system. There is no mammography there, other than at the hospitals."

"It was enlightening that not a whole lot [of the workshop content] had really gotten through to them, and that [the teaching methodology used] wasn't really the best approach ... [what] we had done for the previous day and a half for the nurses."

"It's an acculturated lifestyle, and its just the way it is, and as much as that feels not OK for us in our Western culture, it's everything about theirs' ... when I'm there, my watch is off, except for clinical work, ... that's one thing I instill in the students, is that if you're scheduled to eat at 12, plan on 2, and so, I appreciate that, but I ... soon forget when I'm back home.... I'm going to be very careful how I write my limitations [for funded research in Ghana], because I'm not sure that the lack of being able to adhere to a timeline, it's more my limitation than it is 
theirs, because I didn 't..." [build the different time interpretations into the study timeline].

'You ... can't expect that everybody will have read something, although they will try their hardest to do it before they come to class, because they're sharing books, but ... recognize that their eagerness to learn is almost overwhelming for faculty."

"Although you may be able in a classroom to have a computer and be able to use PowerPoint, the opportunity to photocopy things for your students is very extreme.... When I was at XXXX ... the students ... owned a photocopier out under a tree, so when it started to sprinkle, everybody from the classroom ... got up, ran out, to carry the photocopier into the hall. I mean there's a uniqueness you won't find anywhere."

\section{Sharing}

The sub-category 'Sharing' captured instances in which participants shared stories or vignettes that demonstrated ways an outsider could develop an understanding of an unfamiliar setting. Themes that emerged from the data revealed instances in which participants shared information from their background (including background work in Ghana), about challenges they had faced, personal experiences, feelings, and personal perspectives. There were 214 pieces of data coded as 'Sharing', with information shared by all 16 study participants in order to help outsiders better understand how to prepare for the setting. US participants shared information about how they strove to understand the realities of the setting.

"One of my main challenges was just to communicate with people."

"I think if you're teaching in a university, all of the students there, because it's so competitive, their language skills are very good; now if you were teaching someplace like the diploma program at the hospital in Kumasi, because they bring in essentially young people from all over that area, many of them are not as well educated."

"If I spoke to somebody in Fante, they looked at me and said ... what did she say? Because I was white, and they didn't expect me to say anything in Fante."

"It's almost like, before you go and teach anything, you need to go and just investigate."

"We were all talking about ... clinical breast exams, the first time I was there, and everybody is talking about it, but I got this weird feeling that they hadn't done $i t$, and I'm making an assumption that they had actually ... done it, and I said 'Well how many of you here have actually done a clinical breast exam on a 
patient?' And there was one. And I said: OK that's it. Come on up, and they're like - What? And I said pull this table up - And I got on the table, and they did it on me ... And I said - we're going to learn this, this is our clinical."

"I had a rather unique opportunity ... because I taught at XXXX when they were starting the nursing program and of course their students come from all over Ghana ... I would take a health behavior or a belief that I had heard, like if your child is not born through the vagina it doesn't have a soul.... I would say how many of you believe that? And about a third of them would raise their hand, and I would say how many of you have heard about that? And a third would raise their hand, and I would say how many of you had never heard of it? And a third would raise their hand, so it gave me an opportunity to test it out, and then I'd ask them where they were from and I could get a feel for mapping out some of the belief systems. It was quite interesting."

"Informal discussions we thought seemed to work so much better, than our formal lectures.... the Ghanaians seem to be more reserved, in sharing their knowledge and their ways of practicing, for one reason or another."

"I realized that as a woman, because I can be rather assertive at times, that that didn't fly too well, so I remember speaking up, because I will do that, and I'm getting - the vibe I got off that - I was like - OK ... I'll not say anything else - I'll just sit here and nod, smile, and I'd write notes and pass them on, and I ended up doing that kind of conduit, so the idea came from someone there, seemed to have been accepted better."

"We told people when we came back - It was like going back into the '60s, [in relation to resource actualities and facilities at the teaching hospitals in Ghana] it was definitely ... like going back to the '60s."

A Ghanaian participant shared his perspective about why it is important for outsiders to develop an understanding of the setting, and work in collaboration with insiders when embarking upon an effort in an unfamiliar setting.

"I think people may have the right intentions, and a very good heart, but that does not necessarily mean that it will work well, if you don't know the proper mechanism to get your point across to the people."

\section{Not Knowing}

The sub-category of 'Not knowing' represented researcher interpretations of US participants' comments regarding issues or concepts they had been unprepared for, or had not understood that it was something they even needed to prepare for. 'Not knowing' captured participant comments that reflected instances in which US participants had 
anticipated and prepared for a concept, but realized after arriving in the unfamiliar setting that they had prepared inadequately, and needed to more fully develop understanding.

Data revealed instances where US participants' initial personalization of information needs proved inadequate and resulted in participants 'Not Knowing' something later. Those instances were based, in part, on experiential knowledge gained from other settings, and an incomplete understanding of the Ghana setting. In such instances participants' first-hand experiences in the setting subsequently identified knowledge gaps, which prompted a re-personalization of information needs to facilitate preparation processes for future experiences.

“...Our first trip, we did not prepare adequately ... we thought we were prepared ... we had gone back and forth via email with the director of the school ... what did they want us to talk about ... we talked about our goals and what our aims were, and we were totally off ... when we got there and realized what a different culture - talk about culture shock! ... I really thought that I'd seen it all, and I was prepared, I know what it's like to be in an unfamiliar setting ... I've been through some culture shocks before ... I've been there, done that ... one of the things was we brought the wrong clothes, it was really hot-just environmentally.... We expected it to be hot but we didn't expect it to be that hot! ... it was 120 degrees .... There were no flushable toilets in the school ... a student would follow you in ... with a bucket of water, and ... pour the bucket of water into the toilet.... One of the things they wanted us to talk about was wound care, so I have done wound care lectures before, we talked about ... venous versus arterial versus neuropathic. Well they wanted to know about buruli ulcers and how we care for infectious wounds... I was like-you need to teach me, it ended up being they were teaching me about infectious ulcers using the resources that they have, we knew that the life span was mid 50 to late 50s, but ... we were just so focused on teaching wound care and how we do it here.... Looking back we learned much more from them than they learned from us.... We just learned so much more about the challenges that they're facing, and how well they do utilize all the resources that are there for them.

\section{Questioning}

The sub-category of 'Questioning' captured instances in which US participants (outsiders) had verbalized confusion about something they had encountered or been unable to gather information while attempting to 'Lay the Groundwork' and 'Develop an Understanding' of the unfamiliar setting. The sub-category 'Questioning' also captured instances in which insiders or outsiders questioned psychosocial interactions and principles related to human rights. Themes that emerged from the data included incidences when participants questioned access, attitudes, fit, interpretations, methods, ethics, and social justice. 
Constant comparative analysis during US interviews enabled researcher identification of the themes that US participants had identified as problematic for them to understand. During Ghanaian interviews, question probes were used to solicit insider's clarification of the concepts that US participants had questioned, thereby facilitating outsider understanding of those concepts. Clarification of questioned concepts represents a feedback loop in the conceptual model of the theory 'Preparing for an Unfamiliar Setting' during which one process step prompted another in the cycle of preparing.

US participants questioned the way things were done in the unfamiliar setting, and expressed uncertainty about attitudes and reasoning processes from insiders and outsiders alike.

"If you just showed up at a place, or even if you tried to call and make an appointment, sometimes you got the run around. People didn't want to talk with you, or they wouldn't."

"I think that people [in Ghana] hold documents really closely. I'm not quite sure of the reason for that, if it's sort of like intellectual property, the idea that they don't want anybody to steal it ... stealing ideas is what I mean by that."

"I really got kind of sick on a conference call the other day when someone who'd been there once said: 'Well I've been to Africa and I understand the difference in time between American time and African time'... I almost died because the person on the phone had studied in England and the States, and he's just as westernized as anybody could be."

[We should be] helping them improve their own system. There's no reason that the nurses there could not be doing the kinds of clinics that we're doing, and we're coming halfway across the world to do this. You start wondering about the ethics of the whole thing in some regards."

"Sometimes, especially the younger they [US students doing a clinical emersion experience in Ghana] are, the harder it is for them to hold back... It's that social justices thing coming out in them, but they have a hard time filtering."

A US participant's comment drew attention to questions outsiders may have when they do not fully understand something that is being done in that setting. The exemplar was interpreted to mean the outsider did not have a full understanding of task shifting for health care delivery in the unfamiliar setting, and raised researcher questions as to whether misinformation or inadequate information may have contributed to outsider perspectives. The exemplar illustrated a situation in which outsider perceptions of what were being done were in conflict with personal beliefs and values.

"The thing that kind of jumped out at me and caused me horror and trepidation was that they were taking lay people and training them in roles, and how to do tasks, and no background but just how to do a task, even surgeries.... They're 
teaching people how to do appendectomies, but if there were any complications, forget about it, they were just trained to do the task, so if anything went wrong you were just dead! So ... I was horrified by the whole idea."

\section{Category 5: Framing the Outsider Role}

The category 'Framing the Outsider Role' captures preparatory processes related to interpretation of the manner in which the outsider's role should be enacted in the unfamiliar setting. The exemplar immediately preceding this section demonstrated the need for outsiders to be able to understand and frame their role in keeping with the realities of the unfamiliar setting. This category signals the culmination of preparatory processes, when outsiders and insiders work together effectively as partners in accordance with global development guidelines and principles. 'Framing the Outsider Role' effectively assumes outsiders have successfully networked, communicated, built relationships, and developed a working understanding of the unfamiliar setting, as earlier processes in their preparation for the unfamiliar setting. It then assumes that outsiders should align their efforts in keeping with global development standards, such as those mentioned by Ghanaian participants and laid out in the Paris Declaration (High Level Forum on Aid Effectiveness [HLF], 2005). Categorical findings have been organized to demonstrate researcher interpretation of the outsider role in keeping with interview data, according to sub-categorical headings that represent effective preparation processes, a) listening, b) following, c) supporting, d) working together, and another sub-categorical heading that represented ineffective processes, e) ineffective partnering.

\section{Listening}

The sub-category of 'Listening' captured the ways outsiders could take note and learn from insiders when framing their role for the unfamiliar setting. Two different themes (two-way learning and seeking insider insights) emerged from the data under the 'Listening' sub-category. US participants revealed instances of two-way learning that enhanced their understanding of organizational structures affecting healthcare delivery, nursing education, and nursing practice in Ghana, thereby facilitating an understanding of the nurse educator role in the setting. US participants talked about how they listened and gained insight from Ghanaian insiders.

"The students were very open, we talked with them informally... What really struck us was their respect for faculty. When we had our discussions on wound care and infection control, we learned a lot from them on how they were taught to do things. When we spoke with the students we said ... Is nursing school hard? ... She said, 'Oh no, it's very easy, you just come to class, you write everything down, and you memorize it' And it hit us ... they really don't have the resources ... they're just coming to class with their paper and pencil and writing everything down." 
"When we went the first time they were promoting ... what they called the Health Scheme, the government health insurance, so we talked a lot about that and the barriers that they faced in getting people to sign up. We just learned so much more about the challenges that they're facing and how well they do utilize all the resources that are there for them."

"I was able to talk with the local doctors that we worked with.... I learn something every time, some new disease I was unfamiliar with, or some medicine.... I love to speak with the local healers, because I find it fascinating, the alternative therapies out there."

A Ghanaian participant revealed insider perspectives that drive the organizational structure of healthcare delivery in Ghana, lending insight for outsider's understanding of the setting, as a backdrop against which their role could be enacted.

"We think that our mission is to ensure a healthy population for national development. We can only enjoy a healthy population for national development if we make healthcare accessible to the people living in Ghana. How do you make healthcare accessible to the people living in Ghana? We can only make healthcare accessible to the people living in Ghana if you have healthcare workers who are actually at the doorsteps of the population, you see health care should be accessible to the people living in Ghana, so if you have a lot of community health nurses they go to the periphery and they look at cases and they give that first care, One: to prevent complications, Two: to signal to the individual that this is a complication to move to the next level, by so doing they are also rendering a critical health care to the people."

One US participant's response summarized 'Listening' as a step in the process of framing the outsider role.

"I really want to emphasize the thing about listening to the people that you're working with, because it's typically the truth that they know what the problems are, and what the solutions are, and they just don't have the resources to implement the solution. So it's really listening to what their priorities are."

\section{Following}

The sub-category 'Following' captured pieces of data that represented areas outsiders should focus on when framing their role for the unfamiliar setting. Themes that emerged under the sub-category of 'Following' included following national needs, priorities, and the strategic plan; existing guidelines, regulations, policies, and procedures; and healthcare system realities. Participants from the US and Ghana drew attention to the need for outsiders to frame their efforts against the backdrop of the country's strategic plan, healthcare priority needs, treatment guidelines, and healthcare system realities. A Ghanaian participant noted: 
"I also like it ... [when] everything falls into the national plan, that's the strategic thinking, our vision, and our mission. So that everything will just enhance what we are doing. [Ghanaian] people will be very happy when whatever is brought touches their reality and then we are motivated too to work."

A US participant noted:

"The Ghana Health Service Strategic Plan ... is very informative ... [and] I use the Ghana Health Service treatment guidelines."

A Ghanaian insider also drew attention to resource realities in the setting, and served to remind outsiders that their role should be framed against that backdrop.

"You can be the best surgeon on earth, if you are sent to a facility where there is no tools to work with you are as irrelevant as someone who doesn't know anything. You have the skills, a case is brought to you, you look at the case, this is something I can manage, but the tools to work with are still not there."

Participants from both sides highlighted the need for outsiders to frame their role in compliance with the existing regulatory practices, professional guidelines, and procedural practices for the setting. A US participant recommended that outsiders should:

"Know... the legal issues involved when traveling abroad, as a provider and as a faculty [member]. What the student role is legally, what the faculty role is legally. As a provider you can't just walk into a country and say I'm here to treat.You have to respect their rules, their nursing practice acts, as far as understanding what liability would be involved if there was a legal issue, as far as malpractice."

Ghanaian participants lent insight to existing regulations and procedural practices that outsiders would need to be aware of in order to frame their role to fit the setting.

"First and foremost, we in Ghana appreciate the fact that nursing is four broad categories for 1) nursing administration, 2) nursing education, 3) nursing research, and 4) nursing practice. So whatever form it takes, it could be public health - it could be pediatrics nurses, whatever. So if you take these four areas, anybody who is trained outside but wants to come and work in Ghana, be it in research, be it in education, be it in practice, or nursing administration, you must go through registration processes to get registered-get licensed. The license can take the form of a temporary one - that is from day one to three months, or a permanent one where you sit-our licensing exam is conducted twice a year.... When it is nursing education, we have a curriculum, various curriculums for various programs. So wherever you will find yourself, you will need to go according to the dictates of the curriculum and it will always be under the supervision of the head of the institution." 
"Collaboration has to do with all our stakeholders. For example if anybody [an outsider] is coming in with any new knowledge or skills, we know nursing is also practice, so we ...form a team or a committee comprising nurse educators, nurse regulators and nurse clinicians, who are in the practice area. We come together, and other relevant stakeholders, we come together and look at the material vis a vis the African content or Ghanaian context and then we use" [it].

A Ghanaian participant with experience as a key stakeholder in development efforts for healthcare in Ghana drew attention to the need for all development efforts to be aligned or framed in consideration to global standards.

"For the Paris Declaration it was agreed ... that when ... partners are coming to developing countries to help, they should behave as health partners, that is the declaration. And what it means is that you will avail whatever you have with the developing nation, and then you will ... give technical support for its implementation.... So that they will be [following] us."

\section{Supporting}

The sub-category of 'Supporting' captures pieces of data that represented ways in which outsiders could frame role interactions to help build and strengthen the capabilities and capacities of insiders. Themes that were grouped under 'Supporting' included a) empowering, b) offering guidance, c) sustaining, and d) not imposing. US participants provided the majority of data that were coded under the sub-category of 'Supporting.'

"I think that there are always challenges in being an outsider... You have to be sincere with people. They have to believe that you're sincere. You have to listen to what they have to say. You can't go in thinking that you know the answers. And you have to really develop a true partnership, and that's not always easy for us Americans, because we always think that we have the answers; but our answer isn't necessarily transferable in the context that we're working in. So I think that you have to be flexible, and I think that the people that you're working with, that you're becoming partners with, have to feel like they are being equal partners, and you're not just there to ram something down their throats."

"There are some experts there... for us to go in and assume that we have more knowledge... If you already have an expert there ... you need to tap into that, because that's very insulting, and it's not appropriate, and you're loosing the validation of what they have in their own country, and I said I really want XXXX to teach that class ... and I fought for that.... she said it changed her life, that she got up there and presented, and got the recognition, and now she's doing her own research study on it."

"We did see that there were other opportunities for us to help them improve their own system as opposed to relying on outside help." 
"Respecting the local healers, the local providers, never coming in and trying to show them up, and saying the Western way is the best way, because once you leave, they are the only ones left there to help, and ... if you disrespect them, and the community sees you disrespecting them, then they loose face or honor... so you've got to come in and help them, with providing the best care, and showing the ways that we would do things, but also integrating it with the way they do things.... You need to find out what's available there ... make sure they have what their resources are and then be able to promote the resources from within." "If something is not harmful [traditional healing practices], leave it alone! If something is harmful then you try to find out what you could substitute for that practice; and, things that are helpful, to support them."

"Know... what they have access to in their own culture and country, so that we don't take the Western high-end view, thinking that we're going to implement all of that there, because it's just not going to be possible."

"We took books the first time ... and we took books the second time. We also ... sent some computers and ... fix[ed] it so they had access to our library on the Internet."

A Ghanaian participant reminded outsiders that efforts should be realistically designed to work in the realities of the setting, so outsiders can support what is already being done.

"Match your skill with the type of facility you are going to if you can, actually be relevant to that facility."

\section{Working Together}

The sub-category of 'Working together' captured pieces of data that illustrated ways outsider roles have been framed in keeping with global development principles cited under the process of 'Following'. 'Working together' was demonstrated through participant responses that were organized under the themes of a) collaborating alongside one another, b) making adaptations, c) respecting one another, d), referring to locals, and e) relying on insiders.

Ghanaian participants talked about how they had worked with outsiders.

"We have our good friends from $X X X X$, they said, Look, we have these goodies ... and we want to share with you. We went through the processes, and to cut a long story short, experts have come from $X X X X$, they met with regulators, clinicians, tutors, and we fine-tuned the program they had brought to suit the Ghana context.... We went into a three day residential retreat ... broadened it up, and when we finished it was fantastic." 
If you have certain best practices that you ... want to integrate, you share it with us. Then we see it, we look at how we can customize it to fit ... the Ghanaian context. So we do not just take something from the developed countries and just integrate it into our communities. No, we look at how best we can integrate it and if the desire is, we integrate."

"By regulation you can only register temporarily for the 1st time and the 2 nd time. But ...for one of the lecturers, we had ... a 3rd time ... So, the regulation is there, but sometimes we add the human face to it."

US participants talked about how they had purposefully worked with insiders.

"With our clinical work we're doing active exchanges with the school of nursing, so our health worker students are working alongside their health workers."

"If we found someone with diabetes ... we sent them out there for care, we always went and visited the folks in the hospital, they always knew we were in the community, we always used them for x-rays or lab tests when we felt that that was necessary."

"We have a very good relationship with the health system, so as people come in who have chronic conditions we refer them to the National Health Service."

"They [NMCG practice regulators] are willing to negotiate. I went in because one of their requirements was that you practice 6 weeks on a ward before you get licensed, and I can understand why they would do that, but what I did was negotiate with them, because I'm a nurse practitioner... I said, you know I would have difficulty on the wards in the US because that's not what I practice, I practice primary care, and I had the level of technology that was evident on their clinical ward to teach, so they were perfectly willing."

Outsider's reliance on insiders was another aspect of their role, representing instances when insiders provided access to information resources, key informants and gatekeepers, in-country transportation and accommodations, and served to safeguard outsiders against health hazards in the setting. A US participant talked about her reliance on her Ghanaian contacts. [They] "... picked me up from the airport, they took me out to dinner, they took me shopping." outsiders.

Ghanaian participants revealed their purposeful interactions on behalf of

"If someone would like to be on her own... you offer it up in an open kind of choice for her to have her own accommodation, and ... to make sure that at least she'd have water, potable water." 
"There were ... documents that were given to them from the Human Resources Development Division $[\mathrm{MOH}]$ or the Director."

"You come to work in the country and therefore we have to introduce you to the regulatory body."

A Ghanaian participant summarized concepts for outsiders to consider when framing their role and working together effectively in an unfamiliar setting.

"We have different levels of development, and of course the truth also is the fact that [if] one is not understanding [another] one's point of view does not necessarily mean that ... [the different view] is not right. Many do that. So it all boils to understanding, cooperation, collaboration, respect, maturity."

\section{Ineffective Partnering}

Data revealed circumstances when outsiders could be 'Ineffective partnering' while 'Framing the Outsider Role', and enact it inappropriately by failing to understand, cooperate, collaborate, respect one another, or display maturity in actions. Those instances represented the opposite spectrum from the first four sub-categorical headings, and were linked to ineffective networking, inadequate or ineffective communications, failing to build relationships, and an inadequate understanding of the unfamiliar setting, when earlier steps in the process of 'Preparing for an Unfamiliar Setting' proved inadequate. Outsider's recognition of ineffective processes could prompt a return to the earlier preparatory processes and eventually enable an effective outsider role if preparations were expanded adequately. Findings for 'Ineffective partnering' were grouped thematically as a) not listening, and b) not working together. A US participant shared her experiences after returning from Ghana, when she was attempting to help guide another individual who was embarking on a similar endeavor.

"I gave feedback after that visit ... that they need[ed] hands on [demonstrations and return demonstrations], ... the woman who followed up on me didn't do it, she said, No, No, No, No, No, No.”

Ghanaian participants shared experiences that were interpreted as 'Ineffective partnering'.

[Some outsiders] “... want to play a frontline role to implement.... Recently I was in Denmark, and I was told that when they come we should tell them that it is technical assistance they should give. They are health partners, they are not implementers."

"The big problem we ... have is that a health partner will come and ... sometimes we don't know how the proposals are written. Because they [funded grants] all emanate from the way the proposal was written, and as a result the granting 
coming in, because if the proposal didn't cover that area, ... the grant will not let us ... [align our goals] And so, applying the budget becomes very difficult."

"It is only XXXX that is trying ... the program, but they have not been recognized, because there is no ... establishment for it. When they started the program they did not inform the Ministry of Health to ... give them an establishment.... They came from $X X X X$... to start the program, and they didn't have any [approval from regulatory agencies], the Ghana government wasn't aware of it. So that ... [program] is not working in the [Ghanaian nursing education] system."

"If it's wrongly done, in the first place you put yourself in danger... nobody protects you. You don't have the government or the authority to do that. [Outsiders] ... shouldn't assume that everything will be well."

A Ghanaian participant shared an example of what can occur when outsider preparations have not adequately provided an accurate understanding of contextual realities. The example demonstrates a misalignment of value and belief systems in which 'Ineffective partnering' resulted.

"Something happened for her, she said she was not a Christian, and so it was difficult for her to.... Adjust to some of these things, because in this university we have ... integration of faith and learning ... as you are teaching whatever course, [you bring your faith into it].... And another issue ... had to do with adjustment to local ...food, she felt that some of her needs were not being met by the institution.... she wrote a letter ... that was given to the administration ... she left on a Sunday, it was not on a working day... It was difficult ... she was in town somewhere for about three days before she left for the United States. We tried to contact her, she refused."

Regardless of the way it was enacted, 'Ineffective partnering' arose from outsider's being inadequately prepared for the unfamiliar setting they were going to. A graphic illustration of theoretical concepts for 'Preparing for an Unfamiliar Setting' is provided in the next section, as a theoretical model to facilitate understanding of the various processes that can occur when outsiders embark upon such an endeavor.

Feedback loops are included in the theoretical model to indicate instances when outsiders may have needed to return to an earlier process step for remediation as a new knowledge gap or instance of 'Ineffective partnering' was recognized. In instances where outsiders failed to recognize 'Ineffective partnering,' their preparation processes became stalled at earlier stages, and they were not able to fully develop their outsider role, nor frame it according to global development expectations.

\section{Summary of Study and Emergent Theory}

The purpose of this study was to generate a grounded theory to guide aspiring nursing faculty in developing the necessary knowledge base to fulfill the nurse educator 
role in an unfamiliar setting. A substantive theory, 'Preparing for an Unfamiliar Setting,' was generated from researcher interpretation of findings that emerged as participants' insider perspectives and tacit knowledge were explored. Exploration revealed the processes whereby outsider nurse educators had gone about acquiring the necessary knowledge base to fulfill their nurse educator role in the unfamiliar setting. Theoretical concepts that emerged and were coded as various steps in the process of 'Preparing for an Unfamiliar Setting' can serve to guide other nurse educators embarking upon similar endeavors, since those concepts represent relational tacit knowledge that can be shared, understood, and interpreted by members of the nursing education community.

A brief overview of the major findings related to the processes that emerged from the categorical data is provided next, including researcher interpretations of the meaning behind each category, to facilitate understanding of the process steps that were recognized during data deconstruction and reconstruction. After the overview, the theoretical model is provided, as a visual representation of theoretical concepts throughout all phases of the process while 'Preparing for an Unfamiliar Setting.' The theoretical model includes representation of feedback loops during the process steps, when outsiders recognized a new knowledge deficit that prompted re-exploration of earlier process steps; or when outsiders were ineffective in partnering efforts thereby necessitating re-exploration of earlier process steps in order to move forward. The theory that was grounded in this study's data revealed that 'Preparing for an Unfamiliar Setting' has sequential steps to the process; but, each outsider's baseline knowledge level, past experience, and existing network served as antecedents to the various steps in the process, and individualized their trajectory along their path to preparation. Study findings that revealed participants' recommendations that can serve to guide other nurse educators through similar preparation processes will be discussed in Chapter 5 and synthesized in table format for quick reference regarding key information resources, outsider information needs, barriers, and facilitators.

\section{Overview of Study Findings}

First and second cycle coded data segments that had been deconstructed from interview transcripts were repeatedly examined, rearranged, and reconstructed during theoretical coding until they were logically organized around the central overarching category 'Preparing for an Unfamiliar Setting'. Theoretical coding was used to further develop five categories that emerged from the data, and combined to generate an explanatory theory that intuitively felt right. The first three categories (steps 1-3) represent preparation processes in advance of travel to the unfamiliar setting. The last two categories (steps 4-5) represent fine-tuning of preparation processes to fit the realities and needs of the setting. Analysis revealed that steps 1 and 2 included data from only the US participants, as they began their preparation processes. Data from US and Ghanaian participants provided insight to steps 3-5, when outsiders were finalizing preparations before travel to the unfamiliar setting, and while they were in-country, continuing to refine their preparations with the help of insiders so they could structure their preparations to fit the setting. 
Researcher reflections prompted the realization that each of the five categories could be represented by focused questions that an outsider might ask to summarize and guide each phase of the preparation process. Brief summaries of each of the five process steps are presented next, along with focused questions that help explain what occurred during each step of the preparation process. In each of the focused questions 'I' refers to the outsider who is preparing for an unfamiliar setting.

All of the US participants $(n=10)$ began their preparation processes (step 1) by 'Personalizing Information Needs'. The focused questions that seemed to guide preparation processes for step 1 were: 'What do I know'; and, 'What do I think I need to know?' US participants were either 'Drawing on experience' or 'Breaking new ground' as they individually determined what they already knew, and needed to know to begin their preparation processes.

The next step (2) in participants' preparation processes involved 'Seeking Information'. The focused question that represented preparation processes for step 2 was: 'How do I get the information I need?' US participants $(n=10)$ sought information by 'Networking' with select individuals as information resources, and by 'Searching for information' via online and print resources. US participants who had been 'Breaking new ground' expressed uncertainty about networking and searching, and indicated they didn't know who to contact, or what they needed to know when they initially started their preparation processes. They also indicated they were challenged to network effectively in order to gather the information they needed. Participants who had been 'Drawing on experience' displayed varying levels of expertise and baseline knowledge as antecedents to step 2 that facilitated preparation processes while 'Seeking Information'.

The third step in the process of 'Preparing for an Unfamiliar Setting' included data that were categorized as 'Laying the Groundwork.' The focused questions that guided preparation processes for step 3 were: 'How do I know what is important to know?' and, 'Where do I go to get the information I need?' Participants that had been 'Breaking new ground' expressed uncertainty about identifying the necessary information, where and how to access the information, and what to expect when beginning to build a partnership relationship. Participants who had been 'Drawing on experience' and already had pre-established networks, pre-existing relationships incountry, and an existing knowledge base of information needs and available information resources were at an advantage in their advance preparations before travel to the unknown setting.

Step 4 represented continued preparation processes that outsiders underwent after traveling to the unfamiliar setting. 'Developing an Understanding' of their role encompassed preparations in consideration to the realities and actualities of the unfamiliar setting. Focused questions that guided this step of the process included: 'How do I make use of what I've found?' and, 'How do I correct gaps in understanding?' Ghanaian insiders were key information resources during this step of preparation, as they clarified and shared information, answered questions, and helped define things that were unknown by outsiders. US participants also shared information, and enhanced their own 
understanding through experiential knowledge and reflective thought processes to critically think about what they had read about and seen, then interpret it in light of the setting. While 'Developing Understanding' and experiencing the setting, outsiders who had been 'Breaking new ground' realized the limits of their advance preparation, and recognized the presence of information needs in response to newly recognized gaps in understanding. Outsider participants who had been 'Drawing on experience' and successfully completed steps 2 and 3, but had not been to the unfamiliar setting before, began step 4 thinking they had a beginning understanding of what to expect (based on past experiences in other unfamiliar settings). Participants who had drawn on experience (but not been to a setting like Ghana) realized that realities and actualities of low resource settings like Ghana can be vastly different from experiences and expectations in other unfamiliar settings, and were prompted to re-visit earlier preparation processes. Participants with first-hand experience in sub-Saharan African settings were more easily able to transfer some of their existing understandings to the Ghanaian context.

The fifth and final step of 'Preparing for an Unfamiliar Setting' involved processes that were 'Framing the Outsider Role.' Questions that focused preparation processes during step 5 were: 'How do I connect the pieces to fit the setting?' and, 'How do I operationalize my role?' 'Framing the Outsider Role' in an effective manner represents the culmination of the process, and pre-supposes that previous preparatory processes have been completed successfully. Step 5 represents processes that have equipped outsiders to advance to an active partner role in the unfamiliar setting. After outsiders have successfully framed their role, a feedback loop remains for ongoing and future endeavors in the setting, whereby experiences from the initial preparation processes are drawn upon for future preparations. Of primary importance while 'Framing the Outsider Role' is the need for outsiders to adhere to global development policies for insiders to drive the partnership endeavor, and for outsiders to simply function in a support staff or advisory capacity. 'Ineffective partnering' captured instances of outsiders who where not successful 'Framing the Outsider Role' in keeping with global development policies. When that situation arose the outsider could return to earlier preparation processes to learn what was needed, or stop and not advance further toward partnership goals for the unfamiliar setting.

\section{Theoretical Model}

Figure 4-1 illustrates the substantive theory generated by this study. Process steps are displayed sequentially, with large arrows to indicate forward movement through the five steps in the process of 'Preparing for an Unfamiliar Setting.' Smaller arrows represent outsiders' re-visiting earlier steps in their preparation processes as individually needed. Instances in which outsiders recognized a gap in knowledge or information, and instances in which outsiders had insufficient understanding or ineffective preparation are represented as italicized text in the theoretical model. Any of those instances could serve to prompt outsiders to re-visit earlier preparation processes. Experiential knowledge gained from each step of preparation can be drawn upon to enhance understandings, thereby speeding up various steps in the process in conjunction with antecedent 


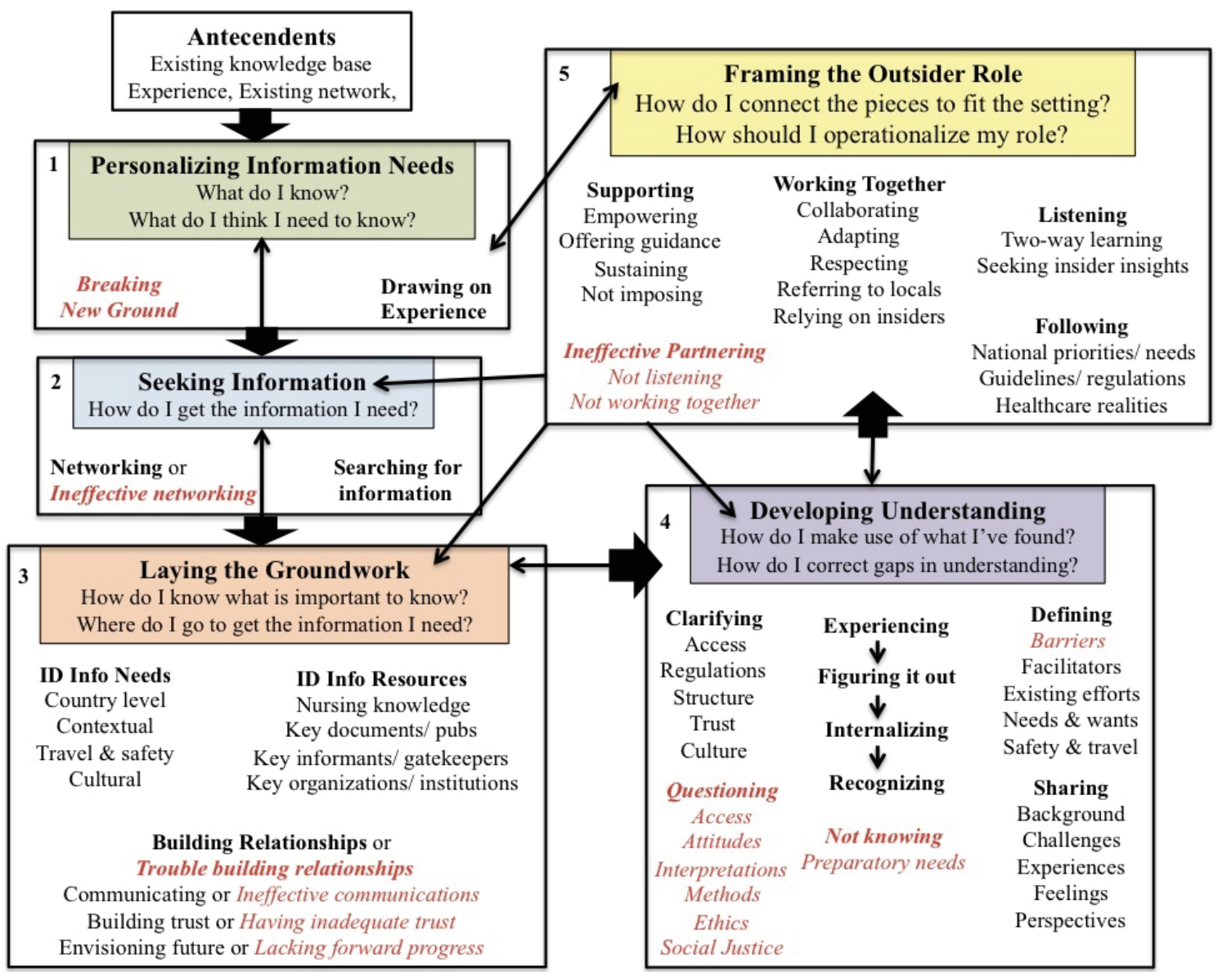

Figure 4-1. Substantive theory: Preparing for an Unfamiliar Setting

Note: Italic red text indicates information gaps or ineffective preparation that prompted a return to earlier preparation processes. 
characteristics (past experience, existing network, and existing knowledge base). After outsiders have successfully completed all five steps of the preparation processes their experiential knowledge from preparing can be used to draw upon for ongoing and future endeavors in the unfamiliar setting. 


\section{CHAPTER 5. DISCUSSION}

The discussion of findings is opened by comparing and contrasting findings from this study against literature evidence and preliminary research that had been presented in the background to this study (see Chapter 2). Study findings are then compared to evidence from recently published literature. Next, this study's findings are discussed in conjunction with prevailing global development policies and guidelines for partnerships in low resource settings.

Implications and recommendations for practice, theory development, and future research are discussed next. Study findings that captured participant recommendations to guide other nurse educators in similar endeavors are included in the discussion on recommendations for practice. Key information resources, outsider information needs, barriers, and facilitators revealed during participant interviews are presented in table format as a quick reference for practice application.

The discussion continues with an evaluation of the quality of the study as compared to criteria set forth by Corbin and Strauss (2008) for research using their methodology. The section includes researcher acknowledgement of the strengths and limitations for this grounded theory study. The discussion of study quality includes researcher perspectives regarding transferability of study findings and theory to other settings. The chapter ends with a discussion of researcher interpretations and conclusions in relation to the research purpose and what this study adds to the body of evidence and professional knowledge base.

\section{Discussion of Findings}

\section{Background Evidence}

Researcher interpretations of findings and comparison to existing literature are based on personal antecedent characteristics as revealed in this study, and consistent with grounded theory methodology (Corbin \& Strauss, 2008). Combined, my knowledge base, experience, and network have shaped the lens through which I have examined and interpreted the data. Findings from extensive literature reviews and preliminary research while gathering background information for this grounded theory study essentially represented my own experiences and knowledge acquisition processes while I was 'Preparing for an Unfamiliar Setting' and progressing through the five steps of the process. I purposefully focused on participant responses during data collection and analysis, and attempted to hold my own experiences at bay. The theory that emerged reflects the participants' experiences, and can now be examined against the background evidence and my experiential knowledge as presented in Chapter 2, while I was 'Laying

the Groundwork' for this study about nurse educator experiences in an unfamiliar setting. 
Nurse educators in unfamiliar settings. This study's findings regarding preparatory learning needs by outsiders going into an unfamiliar setting paralleled recommendations to learn about the social and cultural contexts of the setting (Desantis, 1995; Harrison \& Malone, 2004; Haussler, 2006; Lange \& Ailinger, 2001; Shah, Robinson, \& Enezi, 2002; Wright, Zerbe, \& Korniewicz, 2001), educational system and institutional resources (Byrne, 1998; Desantis; Haussler; Johnson, Ghebreyohanes, Cunningham, Kutenplon, \& Bouey, 2007; Lange \& Ailinger; Melby, Dodgson, \& Tarrant, 2008; Tlou, 1998), other existing efforts (Desantis), technological capabilities (Shah, Robinson, \& Enezi); nursing roles and scopes of practice (Kuehn et al., 2005), and socio-political influences on health care (Girot \& Enders, 2004; Wright, Zerbe, \& Korniewicz). Partnership actions that are mutually beneficial and demonstrate respect for one another (Shah, Robinson, \& Enezi), and developmental aid geared toward national needs of the host partner and capacity building efforts (King, 2009; Wright, Zerbe, \& Korniewicz) were likewise reported in step 5 of the preparatory processes that framed the outsider role in the theory that emerged from this research.

Health in the unfamiliar setting. Literature findings while raising my own understanding of health disparities in the unfamiliar setting (Figure 2-1) reflected concepts that were repeated by participants in this study. Emerging concepts confirmed previous literature describing the need to explore factors influencing health of the population in an unfamiliar setting. Such factors included existing resources, environmental factors, socio-cultural context, socio-economics, socio-political factors, traditional practices, different skill sets for different healthcare workers, nursing workforce realities, and healthcare education needs for the setting.

This study highlighted changes in nursing workforce issues in the unfamiliar setting since the literature review on health disparities (Patterson \& Russell, 2008). Brain drain and lack of leadership development were findings from the literature review in 2008. Ghanaian interviews and informal discussions during this study's fieldwork revealed governmental efforts to reduce the brain drain from Ghana by increasing wages for nurses, and focused efforts for leadership development across all disciplines, including nursing. Several high-ranking officials at the $\mathrm{MOH}$ and its' agencies were enrolled or had recently completed leadership courses at a university in the capital city, through classes taught by the gatekeeper for this study.

A Ghanaian nurse educator and nursing program administrator recounted an instance that demonstrates 'Ineffective partnering,' in contradiction to earlier reports. International nursing education collaborations at three of the four public universities in Ghana had been revealed through the literature (Patterson \& Russell, 2008), and reported in Chapter 2. Information shared during the Ghanaian interview indicated that host and outsider partners in one of those three partnership efforts had failed to involve regulatory bodies in Ghana during the planning stages for the nursing education program they had developed. Consequently, it was reported that the program has not been accredited or recognized by the government as a valid nursing education program in Ghana. Awareness to potential bias in interpretations, and the lack of documented evidence to substantiate the occurrence or capture the perspective of partners involved in that occurrence 
demanded caution in reporting. Nonetheless, the reported instance illustrates potential ineffective preparation processes when outsiders should be 'Developing Understanding' of the unfamiliar setting (step 4), and 'Working together' with insiders while 'Framing the Outsider Role' (step 5).

Societal factors and cultural understanding. Background understandings of societal factors relevant to developing cultural awareness (Figure 2-2) were validated by societal considerations that emerged from this study. Findings from this grounded theory research extended literature findings and revealed that outsiders should prepare for an unfamiliar setting by learning about socio-political, socio-economic, socio-cultural, and socio-geographic factors for the setting, while striving to develop cultural humility rather than simply cultural awareness. Josepha Campinha-Bacote's theoretical principles were cited by participants in this study, as a resource while 'Preparing for an Unfamiliar Setting,' in an ongoing process of "becoming culturally competent, not being culturally competent" (Camphina-Bacote, 2012, ๆ 1). Participants likewise used the phrase "cultural humility' when describing cultural preparation processes and attitudes for outsiders to have. Tervalon and Murray-Garcia (1998) introduced the concept of cultural humility as a mindset to be fostered and developed during healthcare education training programs. They defined cultural humility as "a process that requires humility as individuals continually engage in self-reflection and self-critique as lifelong learners and reflective practitioners... To develop and maintain mutually respectful and dynamic partnerships...." (Tervalon \& Murray-Garcia, p.118).

Preliminary research. Ethnographic findings revealed environmental, social, and cultural considerations related to a) the country of Ghana, b) nursing practice and education in Ghana, and c) institutions associated with nursing education and practice in Ghana. Participants in this grounded theory research revealed needs for outsiders to a) get a big picture understanding of the country that is unfamiliar, b) understand the structure of nursing education, the scope of nursing practice, and practice regulations for nurses, and c) learn about health care and institutions, regulatory bodies for nursing, and governmental agencies associated with health in the unfamiliar setting. Concerning the big picture context of the country, this study's findings drew attention to the low-resource realities of the setting, and hinted at regional variations in resource allocations. Grounded theory findings failed to illuminate urban-rural inequities or north-south regional inequities that had been revealed by preliminary research during ethnographic fieldwork. A possible explanation for the discrepancy is that Ghanaian sampling during the grounded theory research was limited to participants and fieldwork near the capital city of Accra, and most of the US participants' Ghanaian exposure were limited to a specific partner location; while research during ethnographic fieldwork encompassed researcher experiences and observations in 6 of the 10 regions of Ghana, as well as interviews with participants in rural, urban, north, and south locations.

In 2008, ethnographic findings revealed a low public perception of nurses in Ghana, and suggested that the low perception may have been associated with lower senior secondary exam scores for nursing school admission versus those required for university admission. Findings from this study revealed a change in professional context 
for nursing in Ghana that was reflected by a reported increase in the public perception of the profession. Nursing has been transitioned to tertiary level education, rather than the level of a training program, with entrance requirements as stringent as university admission requirements. Informal discussions with my Ghanaian host and gatekeeper revealed that applications for nursing education programs ranked $3^{\text {rd }}$ in the nation as compared to other educational offerings. Combined with an increase in nursing wages, the changes have made nursing one of the more desirable professions in the country, while structuring admission requirements to attract the most qualified candidates.

This study expanded understandings of institutional contexts and health system structures in Ghana, beyond understandings from preliminary research. A Ghanaian participant shared information about a third teaching hospital in the Northern part of Ghana, in the city of Tamale. That information represented a change from the two teaching hospitals discovered in 2008, and highlighted the need for outsiders to be mindful of change and growth in unfamiliar settings.

This grounded theory research specifically sought to understand issues that had been study limitations during preliminary research. Purposive networking while 'Preparing for the Unfamiliar Setting' following the ethnographic study helped build relationships and trust thereby facilitating access to key informants and key information resources for this study. Preparing for fieldwork a second time in Ghana illustrates the ongoing learning and cyclical preparation processes for subsequent encounters in a setting that had been unfamiliar, and demonstrates an outsiders' advancement from 'Breaking new ground' as a novice in step 1 of preparation processes to Drawing on experience' the next time they are preparing to return to the setting.

\section{Recent Evidence}

This grounded theory research revealed the need for outsiders to frame their role (step 5) to support and empower host partners, in a two-way exchange of information that demonstrates mutual respect, and collaboration. The findings were consistent with a recent literature synthesis. Kulbock, Glick, Mitchell, and Greiner (2012) synthesized literature evidence from international nursing education experiences that were focused on study abroad and service learning opportunities in global health. Their review of 23 publications spanning 2003-2010, revealed needs for models to guide the practice of international partnership efforts, and focused efforts to promote authorship and active involvement of host partners through two-way exchanges that are mutually beneficial to host and outsider partners.

Processes while 'Preparing for an Unfamiliar Setting' were consistent with input factors during the engagement phase of Leffers' and Mitchell's (2011) grounded theory "Conceptual Framework for Partnership and Sustainability in Global Health Nursing" (p. 91). They identified baseline factors for outsider and host partners that were essential to the partnership process. Both studies revealed that outsider knowledge about the unfamiliar setting (including socio-economic, socio-environmental, socio-cultural, and 
socio-political aspects), coupled with outsider attributes, expectations of both partners, and insider wants and needs were essential elements to the preparation process. Other commonalities for partnering recommendations across the two studies included needs for mutually defined goals, relational trust, sustainable project leadership, insiders' driving project interventions, and collaborative efforts that are geared toward capacity building and empowerment of host participants.

Recently, partnership experiences between the University of Michigan, Ghanaian universities, and the government of Ghana were shared as a guide for developing collaborative relationships that are specifically geared toward strengthening and training HRH. The collaborative model they developed had four key components: 1) HRH priorities of the host country as a framework, 2) policy decisions regarding HRH should be informed by data from research findings, 3) the realities of HRH education and training needs in the setting, and 4) the research infrastructure for HRH should be expanded and strengthened (Lawson, 2010). Their model supports the findings of this research, particularly steps 4 and 5 of the theory Preparing for an Unfamiliar Setting, when outsiders are working with insiders to better understand the realities of the unfamiliar setting, and framing the outsider role to focus on the priority needs of the host country, strengthen, and support insiders as the program implementers. The report (Lawson, 2010), summarized at a meeting, and housed on an organizational web site, supports this study's findings related to hidden knowledge that can be buried inside organizational or institutional confines. Their work could have served as a valued information resource during this study, however I was not aware of it until my dissertation defense, when it was brought to my attention. Despite in-depth literature reviews and weekly Pub Med alerts to new publications in the field, this report had remained 'hidden' from this researcher, and was revealed through 'Networking' with human information resources (part of the process in step 2 of the theory). Instances of 'hidden' information such as this further substantiate the need for ongoing 'Networking' as a key information resource when Preparing for an Unfamiliar Setting.

In a recent seminal publication (Frenk, et al., 2010), a global "Commission on the education of health professionals for the $21^{\text {st }}$ century" (p. 1927) developed a systems framework for health education reform to meet global needs. They recognized people within educational and health systems as those who would drive needs and demands based on the realities of their existing systems. The Commission acknowledged the significance of social determinants on health, and the need for health providers to become knowledge brokers to facilitate translation of knowledge into evidence-based practice that is relevant to the social context. Their report drew attention to educational constraints in low-resource settings that are imposed by a scarce supply of qualified faculty members for health care education. They recommended exploring short-term placement of qualified teachers from high-resource settings, sharing open education resources via information communication technologies coupled with capacity building efforts, and mutual learning to promote a global perspective among health professionals. Theoretical concepts and study findings revealed while 'Framing the Outsider Role' (step 5) are congruent with the Commission's report and global development expectations for outsider partners. 


\section{Global Development Expectations}

Findings from this study revealed the need for outsiders to frame their role (step 5 of Preparing for an Unfamiliar Setting) in conjunction with existing global policies and guidelines for international development efforts. The Paris Declaration (HLF, 2005) was identified as a key document for outsiders to use while framing their role for an unfamiliar setting. When viewed historically, the Paris Declaration fits into a bigger picture context enabling outsiders to better understand principles guiding international development efforts. Aid effectiveness has been a focus for global development efforts since 2002, when the First High Level Forum outlined aid effectiveness principles in the Rome Declaration (HLF, 2003). After that, the Second High Level Forum on Aid Effectiveness laid out five practical principles to guide the effectiveness of developmental aid in The Paris Declaration (HLF, 2005). Those principles specified 1) host countries as the owners who should drive partnerships, 2) donor countries aligning their efforts with host country goals and existing systems, 3) harmonization of efforts to avoid duplication, 4) measurable results and focused endeavors, and 5) partners who are mutually accountable for results. The Third High Level Forum on Aid Effectiveness took place in Ghana in 2008, suggesting why Ghanaian insider participants were so attuned to developmental principles set forth in The Paris Declaration. The Accra Agenda for Action (HLF, 2008) targeted three areas for improving aid effectiveness as a follow up to The Paris Declaration. Those three areas stressed the importance of 1) host countries as the primary partner and owner, with the right to decide development processes as needed in their country, 2) full participation by all partners, and 3) developmental aid must deliver measurable results. The Accra Agenda for Action (HLF, 2008) focuses on capacity building developmental efforts.

More recently, the Fourth High Level Forum on Aid Effectiveness took place in Busan, Korea, in late 2011. The outcome of that HLF meeting was the Busan Partnership for Effective Development Cooperation (HLF, 2011a). It provides an agreed upon framework for developmental aid that fine tunes years of practical experience and research evidence into a working document to hold partners accountable for their actions. In June 2012, following the HLF meeting in Busan, a steering committee was appointed to monitor the Busan commitments. Discussing the Busan Partnership as it relates to Ghana, the Ghanaian Minister of Finance noted that following the Paris Declaration (HLF, 2005) and Accra Agenda for Action (2008) the government of Ghana developed the Ghana Aid Policy and Strategy: 2011-2013 to formalize their country's stance on foreign aid and country ownership of developmental aid efforts (HLF, 2011b). The Minister further noted that Ghana recently created a framework to assess development partners performance as a tool to track partner adherence to mutually agreed upon goals for developmental aid. In combination with recent legislative acts, and active participation as a member of the working party on aid effectiveness for the HLF, Ghana has poised itself as an active, informed host partner for international development efforts (HLF, n.d.). 


\section{Implications and Recommendations}

\section{Practice}

Implications for nursing practice that arose from this study's findings involved raising awareness to outsiders' information needs, key information resources, and lessons learned from past participants regarding barriers and facilitators they experienced while 'Preparing for an Unfamiliar Setting.' Practice implications while 'Developing Understanding' (step 4) and 'Framing the Outsider Role' (step 5) revealed attitudes and mindsets necessary for outsiders to internalize as they are preparing themselves for role enactment and effective partnering in an unfamiliar setting. Those mindsets reflected study findings indicative of prevailing global development guidelines, and included practice implications that would necessitate outsiders learning about existing efforts in an unfamiliar setting.

Outsiders' information needs. Participants in this study identified key information needs for outsiders going into an unfamiliar setting. The information needs they identified can be used to guide outsiders who are 'Breaking new ground' while 'Preparing for an Unfamiliar Setting' by focusing outsiders' information searching processes to key topic areas that are important for them to understand. Identification of key information needs can guide outsiders as they ask 'How do I know what is important to know?' Table 5-1 organizes this study's findings related to key information needs, and captures those findings in a quick reference format.

Key information resources. In addition to identifying key information needs, findings from this study revealed key information resources that can guide outsiders toward information acquisition, and preparation for effective role development and nursing practice in the unfamiliar setting. Identification of key information resources can guide outsiders as they ask 'Where do I go to get the information I need?' Table 5-2 presents study findings concerning key information resources as a tool for quick reference.

Lessons learned, barriers and facilitators. Participants shared lessons learned, and identified barriers and facilitators to their preparation processes for the unfamiliar setting. Outsiders can gain a better understanding of an unfamiliar setting by learning from the experiences of others, and raising their own awareness to situational and contextual realities, communication patterns, and attitudes that can affect outsider understandings (step 4) while 'Preparing for an Unfamiliar Setting.' Identification of lessons learned can serve to guide outsiders as they ask 'How do I make use of what I've found?' and, 'How do I correct gaps in my understanding?' Table 5-3 provides a quick list of study findings related to lessons learned concerning barriers, and facilitators to outsider preparation processes.

Awareness of developmental efforts. Findings from this grounded theory

research revealed key organizations that can serve as information resources for outsiders 
Table 5-1. Key information needs.

\begin{tabular}{ll}
\hline Information Category & Topic Areas \\
\hline Country level & Big picture, British background, language, burden of \\
& disease, geographic inequities, health needs, country level \\
priorities, country health plan, governmental oversight, & socio-political structure, scope of practice, practice \\
& regulations, licensure requirements, accreditation \\
& guidelines, educational structure, health system structure, \\
& nurse population ratio, nursing curriculum, drug formulary, \\
& existing efforts, national insurance scheme, health of \\
& population, and country wants. \\
Contextual & Resource realities, alternative therapies, traditional \\
medicine, environmental realities (water, sanitation, & climate, Internet, communication technology capabilities, \\
roads), indigenous disease processes, institutional \\
processes, decision making processes, teaching and \\
learning processes, institutional resources, faculty role \\
expectations, infectious diseases, tropical diseases, and \\
global development expectations. \\
Cultural behavior, protocols, time interpretations, cultural \\
taboos, etiquette, role expectations, gender expectations, \\
communication patterns, cultural beliefs, dress code, \\
cultural hierarchy, family structures, and tribal structure. \\
Immunization needs, precautionary measures for health \\
(malaria prophylaxis, well-stocked first aid and medicine \\
kit, bottled drinking water, medical evacuation insurance, \\
disease and injury prevention), food, lodging, and \\
transportation.
\end{tabular}

Note: Identifying key information needs occurred while participants were 'Laying the Groundwork' (step 3) in the process of Preparing for an Unfamiliar Setting. 
Table 5-2. Key information resources.

\begin{tabular}{|c|c|}
\hline Resource Category & Specific Resource \\
\hline Nursing knowledge & $\begin{array}{l}\text { Core nursing knowledge, host nursing program orientation, } \\
\text { cultural nursing theory, diagnostic reasoning process } \\
\text { subjective/ objective assessment, } 7 \text { characteristics of a } \\
\text { symptom, }\end{array}$ \\
\hline Key documents & $\begin{array}{l}\text { National nursing curriculum, code of conduct for nurses, } \\
\text { code of conduct for midwives, clinical schedule books, log } \\
\text { books for students and nursing tutors, } 5 \text {-year Strategic Plan, } \\
\text { National Health Policy, 5-year Program of Work } 2011 \text { - } \\
\text { 2015, accreditation documents, program requirements, }\end{array}$ \\
\hline Key publications & $\begin{array}{l}\text { Local publications (sociology focus, context specific, Twi } \\
\text { phrase book), Paris Declaration }\left(\mathrm{HLF}^{\mathrm{a}}, 2005\right) \text {, global } \\
\text { policies for international development, WHO }{ }^{b} \text { Essential } \\
\text { Medicines, theoretical/ philosophical literature, Where } \\
\text { there is no doctor: A village health care handbook } \\
\text { (Werner, Thuman, \& Maxwell, 2007), } \\
\text { Josepha Campina-Bacote's theoretical work, } \\
\text { Health and culture: Beyond the Western paradigm, and } \\
\text { Healing our differences: The crisis of global health and the } \\
\text { politics of identity by Collins Airhihenbuwa. }\end{array}$ \\
\hline Key informants & $\begin{array}{l}\text { In-house resources (programs abroad, or international } \\
\text { health clinics), in-country experts, cultural centers, past } \\
\text { participants, community health workers, local providers, } \\
\text { natives living abroad, hosts (partners, nursing students, } \\
\text { administration, tutors, MOH }{ }^{\mathrm{c}} \text { officials, NMCG }{ }^{\mathrm{d}} \text { officials }\end{array}$ \\
\hline Key organizations & $\begin{array}{l}\text { WHO, CDC }{ }^{\mathrm{e}}, \mathrm{ICN}^{\mathrm{f}} \text {, Host institution, NGOs }{ }^{\mathrm{g}} \text {, faith-based } \\
\text { organizations, NMCG, MOH, GRNA }{ }^{\mathrm{h}} \text {, Ghana Health } \\
\text { Service, Ghana Health Workforce Observatory, Peace } \\
\text { Corp, Encore Service Corps International }\end{array}$ \\
\hline
\end{tabular}

Notes: Identifying key information resources occurred while participants were 'Laying the Groundwork' (step 3) in the process of Preparing for an Unfamiliar Setting.

${ }^{a}$ HLF: High Level Forum on Aid Effectiveness.

${ }^{\mathrm{b}}$ WHO: World Health Organization.

${ }^{\mathrm{c}} \mathrm{MOH}$ : Ministry of Health.

${ }^{\mathrm{d}}$ NMCG: Nursing and Midwifery Council of Ghana.

${ }^{\mathrm{e}} \mathrm{CDC}$ : Centers for Disease Control and Prevention.

${ }^{\mathrm{f}} \mathrm{ICN}$ : International Council of Nurses.

${ }^{g}$ GRNA: Ghana Registered Nurses Association. 
Table 5-3. Lessons learned: Barriers and facilitators to preparation process.

\begin{tabular}{|c|c|}
\hline Barriers & Facilitators \\
\hline Contextual barriers $^{\mathrm{a}}$ & Human facilitators $^{c}$ \\
\hline Cultural considerations & Big picture contacts \\
\hline Tribal beliefs & In-country gatekeepers \\
\hline Time interpretations & In-country liaisons \\
\hline Language & Liaisons \& hosts \\
\hline Unfamiliar nomenclature & Liaisons, former participants \\
\hline Not following regulations & $\mathrm{NMCG}^{\mathrm{d}}$ contacts $\&$ hosts \\
\hline Understanding health care system & $\mathrm{MOH}^{\mathrm{e}}$ contacts $\&$ hosts \\
\hline Inaccurate understandings ${ }^{a}$ & Personal actions \& attitudes ${ }^{c}$ \\
\hline Misinformation & Learning \\
\hline Lacking cultural humility & Seeking cultural humility \\
\hline Know it all experts & Following, listening \\
\hline Unsustainable interventions & Adapting, referring to locals \\
\hline Access requirements & Relying on insiders, respect \\
\hline Reality considerations ${ }^{b}$ & Communication realities ${ }^{\mathrm{c}}$ \\
\hline Costs for distance partnership & Awareness \\
\hline Time limitations of partners & Collaborative planning \\
\hline Resource \& environmental realities & Sharing wants \& needs \\
\hline Outsider status & Learning language basics \\
\hline Needing trust & Time to develop trust \\
\hline Associated stressors & Anticipating culture shock \\
\hline
\end{tabular}

Notes: ${ }^{a}$ Barriers arising from ineffective preparation from the process theory Preparing for an Unfamiliar Setting.

${ }^{b}$ Barriers arising from reality considerations, not related to advance preparation, from the process theory Preparing for an Unfamiliar Setting.

${ }^{\mathrm{c}}$ Facilitators reflecting effective preparation processes from the process theory Preparing for an Unfamiliar Setting.

${ }^{\mathrm{d}}$ NMCG: Nursing and Midwifery Council of Ghana.

${ }^{\mathrm{e}} \mathrm{MOH}$ : Ministry of Health. 
Preparing for an Unfamiliar Setting. In addition to organizations identified during interviews, findings revealed the need for outsiders' awareness of existing work, to reduce duplication of efforts. Awareness of existing efforts suggests preparation process applications beyond awareness of existing efforts in the specified unfamiliar setting to a broader awareness of existing development efforts in the global community and lessons learned in the international arena. Although participants in this study recommended that outsiders should learn about existing efforts, no US developmental agencies were cited as information resources. Online searching during preliminary research in advance of this grounded theory research helped identify the United States Agency for International Development (USAID) as the national developmental agency for international aid funded by the US.

Capacity Plus (n.d. a) is a current USAID project that extends work following the Capacity Project to strengthen and equip HRH in low-resource countries to meet MDGs. The Project's final report (Capacity Project, 2009) shared knowledge gained and lessons learned as a guide for HRH development efforts. Capacity Plus has an open access online knowledge library to share and disseminate the collection of evidence compiled from the Capacity Project and Capacity Plus (Capacity Plus, n.d. c). Particularly applicable to the unfamiliar setting for this grounded theory research, Capacity Plus began ongoing work in 2010 to support Ghanaian efforts to build their HRH (Capacity Plus, n.d. b), and extended a collaborative partnership that had involved Ghana as a case study for HRH practices during the Capacity Project (Capacity Project, n.d.).

Multiple information resources are available through the knowledge library at Capacity Plus. Of particular relevance to this study's research purpose, Learning for Performance: A Guide and Toolkit for Health Worker Training and Education Programs (IntraHealth International, 2007) was the product of partner efforts and lessons learned during the Capacity Project. It details 12 step-by-step instructions for outsiders when developing health education partnership endeavors in low-resource settings, and includes worksheets for planning.

Developmental agencies from other countries can also serve as information resources for outsiders 'Preparing for an Unfamiliar Setting.' Study participants for this research did cite the Canadian International Development Agency (CIDA) as an active partner for development efforts in Ghana. An exploration of their website (CIDA, 2012) revealed that Ghana is one of the focus countries for their developmental efforts. The Overseas Development Institute serves as another information resource. Their country study on Ghana's untied aid (Geddes, Mensah, Natali, \& Quartey, 2009) revealed foreign aid during 2007 going into Ghana from 11 separate nations, and suggesting further evidence of the need for outsiders to be aware of existing work and avoid duplication of efforts when 'Preparing for an Unfamiliar Setting.'

From breaking new ground to drawing from experience. Practice implications arose from this study's findings relevant to an outsider's baseline knowledge and experience level when beginning the process of 'Preparing for an Unfamiliar Setting.' Participants judged to be experts in the field (as evidenced by researcher interpretation of 
demographic characteristics) progressed quickly from step 1 to step 4 of the theoretical model, with shorter, focused preparations during steps 2 and 3 of their own preparation processes. Practice implications suggest that the theoretical model can guide future preparations by making novice and experienced outsiders alike more aware of existing information needs and information resources, thereby shortening preparation processes and equipping outsiders with necessary knowledge and insights to understanding contextual realities and regulations in the unfamiliar setting.

\section{Theory Development and Future Research}

Future plans include sharing the completed theoretical model (Figure 4-1) with host and US participants, and soliciting their review of the research findings in a member check of researcher interpretations and the model itself. Officials at the $\mathrm{MOH}$ in Ghana have expressed a desire to have a copy of the published dissertation and theoretical model, to use as a potential framework to guide preparation processes for future work with outsiders coming into their country for nursing education endeavors. Those interactions represent the potential to test researcher interpretations and the potential to test the model regarding applicability and preparation effectiveness for outsider going into the specified unfamiliar setting of Ghana.

Future research can test the model in other unfamiliar settings. Conducting a Delphi Study to explore expert opinion of theoretical concepts and look for gaps in understanding could expand the theory. Future plans include disseminating the model to a focused panel of nursing experts from the global community with experience in various unfamiliar settings, seeking a consensus on theoretical interpretations, refinement, and validation of the model. A Delphi Study would offer opportunity to generate further ideas, potentially validate other evidence from literature findings and incorporate them into the model that were not included in this study's participant experiences, and build relational tacit knowledge between nurse educators to help guide members of the profession in the global community who may be 'Preparing for an Unfamiliar Setting.' Revised versions of the theoretical model could continue to be tested in unfamiliar settings and continually refined to address changes in the global community.

\section{Study Quality}

Corbin and Strauss's (2008) grounded theory methodology provided the framework for this research. They laid out a set of 10 criteria to use when evaluating methodological rigor and the quality of findings for studies that used their methodology. Those 10 criteria include 1) fit, 2) sensitivity, 3) evidence of memos, 4) context, 5) logic, 6) variation, 7) applicability, 8) concepts, 9) richness or depth, and 10) creativity or newness. Next, this study's findings are evaluated against those 10 criteria as further evidence of the quality and trustworthiness of the study. 


\section{Evaluation of Study Quality}

Fit of study findings against participant experiences was included participant verification of interview transcriptions (50\% response rate), and informal member checks during interviews to repeat back portions of the conversation and clarify researcher interpretations. Focused efforts by the researcher strove to hold personal experiences at bay, be sensitive to participant words, and allow the data to speak for themselves. Future plans to offer participants the option to member check researcher interpretations of the data and theoretical model have the potential to enhance fittingness of findings. Instances where researcher understandings of personal preparation processes extended beyond study findings were compared and contrasted in the discussion of findings, and not allowed to impede on researcher interpretations of participant responses. Written and voice memos tracked researcher reflections and analytical memos throughout the research and reporting process.

Context played a major role in this study, and findings were reported in consideration to various contextual considerations, as evidenced by outsider information needs relevant to the unfamiliar setting (Table 5-1), key information resources for the unfamiliar setting (Table 5-2), lessons learned, barriers, and facilitators relevant to the unfamiliar setting (Table 5-3). The theoretical model provides evidence of the logical flow of preparation processes, including feedback loops when variations in participants' preparation processes were evidenced due to knowledge gaps, or ineffective preparation processes, and instances when they returned to earlier preparation processes to correct gaps in understanding.

Study findings are useful and applicable as a theoretical model that can guide aspiring nursing faculty toward developing the necessary knowledge base to fulfill the nurse educator role in an unfamiliar setting. This study reveals participant experiences and makes their relational tacit knowledge explicit for others to learn from as they are preparing to go into an unfamiliar setting. Theoretical concepts were explored and developed through researcher interpretative thoughts to capture the properties and dimensions of theoretical categories, in such a way that the concepts would make sense to nurse educators who can interpret and share relational tacit knowledge. Rich thick in vivo coded segments of data were used to illustrate researcher interpretation of theoretical concepts and categorical arrangement of the steps in the process for the theoretical model. Study findings add new understanding to outsiders' preparation processes in consideration to prevailing guidelines for developmental aid and expected partner behaviors in the $21^{\text {st }}$ century. Disseminating the completed dissertation and theoretical model to study participants that requested the publication could afford another opportunity to assess fit of findings against participant experiences and expectations of end users of the theory. 


\section{Strengths and Limitations}

Strengths. Focusing this grounded theory research in the specified unfamiliar setting of Ghana represented strength in support of the study purpose. Personal contacts and an existing network of key informants and past research experience in the setting proved beneficial to this research effort. Past partnerships for nursing education in Ghana afforded opportunity to capture data from a variety of outsiders who had first-hand experience implementing the nurse educator role in the setting. Ghana's active involvement in HLF efforts, policies, and guidelines for international partnerships provided evidence of their expertise in the area, as knowledge brokers who could share understandings with outsiders 'Preparing for an Unfamiliar Setting.' Ghanaian officials' continued interest in this dissertation research and the theoretical model that emerged suggests the potential for an ongoing partnership relationship.

Faculty oversight and committee member expertise represented another strength of this dissertation research. In combination with preliminary fieldwork, those human resources lent credibility to the research. The dissertation chair and $\mathrm{PhD}$ program director each provided faculty oversight at various points throughout the process of study design, data collection, and analysis. The dissertation chair was the primary source of guidance. Committee members served as content experts in relation to various aspects of the research, and critiqued the initial study plan and final report in consideration to those areas of expertise. An outside committee member served as the expert on international nursing education collaborations, and served as cognate course faculty for independent studies that facilitated researcher understandings of background information prior to conducting the ethnographic study. As the deputy director of a WHO Collaborating Center for International Nursing, she exemplified expertise in the area. Another committee member with years of public health experience consulting with post-civil war governments served as the content expert for practical application of the theoretical model to low-resource settings. The fourth committee member was present at the Arista 3 Conferences that targeted understanding of international nursing issues and concerns, completed her own dissertation using grounded theory methodology, and prompted researcher insights relating to administering, monitoring, and regulating nursing education programs. As a nurse practitioner, the last committee member likewise brought a background in public health to research table, as well as expertise in faculty evaluations and developing an evidence-base for practice.

Using Corbin and Strauss (2008) framework for this dissertation research represented another strength to the study. Their methodology guided all aspects of the research, from development of the research purpose and specific aims to the research design, data collection, data analysis, report of findings, and study evaluation.

Limitations. A methodological limitation that arose during this study occurred when plans to have Ghanaian transcriptionists transcribe fieldwork interviews were delayed because the intended transcriptionist was unavailable, and the back-up transcriptionist became ill with typhoid fever. The occurrences drew attention to the need for flexibility and adaptability to contextual realities inherent with working in unfamiliar 
settings. After receiving the completed transcriptions I realized there were several gaps, and had to re-transcribe them myself to ensure accuracy of transcribed content. Delays in transcription delayed data analysis and development of the grounded theory. I had thought I was building in plans and back-up plans to address language limitations that had been recognized during preliminary research in 2008, but came to realize that contextual realities exist and have to be managed for effective work in unfamiliar settings.

Limitations related to being an outsider in an unfamiliar setting were addressed by reliance on a Ghanaian gatekeeper, host, and key informant. He and his family served as human resources to explain and clarify things I may have misunderstood or failed to understand. They also served as guardians of my safety while in Ghana, and provided transportation and interpretative services as needed during fieldwork and cultural excursions that included worship services at two different locations on two separate Sunday mornings, and shopping in local markets, adding to my own cultural understanding of the setting.

Sampling presented other limitations to the study. Theoretical saturation of the data helped determine the end of sampling for US participants, but variation in their experience level revealed limited participants who had been drawing on experience when they began their preparation processes. Using a sample with more extensive experience in the international arena may have yielded other information that could have been incorporated into the theoretical model. Future plans to conduct a Delphi Study with a panel of experts can address this potential limitation. Time limitations during fieldwork affected sampling in Ghana. Repetitive themes were noted after the first 4 Ghanaian interviews, including commonalities with US interviews and common clarifications by Ghanaian participants for issues US participants had not understood. Theoretical saturation of Ghanaian interview data was uncertain. Data collection in Ghana ended after 6 interviews, due in part to travel restrictions that prohibited further interviews. The small sample size represents a limitation. Feasibility considerations for sampling included time for fieldwork, and scheduling that could accommodate my Ghanaian host, the drivers he had arranged, and potential Ghanaian participants. Likewise, geographic location in Ghana was a feasibility consideration. All of the fieldwork interviews took place within an hours' drive of the capital city. It is possible that other Ghanaian participants may have included different insights than those that were interviewed. Future plans to conduct member checks of study findings (including the theoretical model) with Ghanaian participants, combined with the governments' potential use of the model as a resource to guide outsiders coming into the Ghana setting, may help counteract this sampling limitation by verifying and testing the model from the perspective of Ghanaian insiders.

\section{Transferability}

In keeping with principles for qualitative research (Lincoln \& Guba, 1985) transferability of this study's findings to other settings as been addressed by providing a 
rich, contextual description of theoretical concepts and a detailed account of study findings as a mechanism to allow future users of the data and theoretical model to draw their own conclusions about the applicability of these findings in other settings. Constant comparative methods of data analysis and interpretation inherent with grounded theory research guided this study (Corbin \& Strauss, 2008). An audit trail was maintained to track researcher interpretation and data sources throughout the study. Future researchers could explore de-identified study records to determine fit and appropriateness of transferability to another context if necessary.

\section{Conclusions}

This study addressed a gap in evidence to guide individuals as they prepare for nursing education partnerships in unfamiliar settings. Shifts in developmental aid to lowresource settings and prevailing guidelines for partnership endeavors indicated a need for research that could equip outsiders with the necessary knowledge base and understanding to be an effective partner in an unfamiliar setting. This study responded to gaps in understanding regarding cultural contexts for higher education in sub-Saharan Africa (King, 2009); and, provides a model to guide other nurse educators as they prepare for nursing education partnerships in unfamiliar settings.

The theoretical model 'Preparing for an Unfamiliar Setting' represents shared tacit knowledge among nurse educators and healthcare professionals regarding the preparation processes whereby outsiders gained the knowledge and understanding necessary to enact their role effectively in an unfamiliar setting. This study adds to the body of evidence and professional knowledge base for international nursing education endeavors, in the context of $21^{\text {st }}$ century developmental aid policies and guidelines. Although focused on Ghana, Africa, as the specified unfamiliar setting, findings from this grounded theory research have been reported in context to facilitate transferability of findings and the theoretical model to other research settings. Theoretical preparation processes were intentionally interpreted in a broad manner, using Ghana as the specified unfamiliar setting from which examples were drawn, with attention to the potential for global application of the theory by researchers in other unfamiliar settings. 


\section{LIST OF REFERENCES}

Advisory Council for Scientific Research in Development Problems. (1998). Framework for a Ghanaian-Dutch Programme of Health Research for Development. [Publication no. 15]. The Hague, Netherlands: Author. Retrieved December 19, 2012 from http://www.cohred.org/publications/library-andarchive/framework_for_a_ghan_1_147/

Africa Guide. (1996-2008). Retrieved February 14, 2008, from http://www.africaguide.com/country/ghana/culture.htm

Aiga, H. (2006). Reasons for participation in and needs for continuing professional education among health workers in Ghana. Health Policy, 77, 290-303.

Aiga, H., \& Kuroiwa, C. (2006). Quantity and distribution of continuing professional education opportunities among healthcare workers in Ghana. Journal of Continuing Education in Nursing, 37, 270-279.

Aiken, L. H., \& Poghosyan, L. (2009). Evaluation of "magnet journey to nursing excellence program" in Russia and Armenia. Journal of Nursing Scholarship, 41, $166-174$.

Akiwumi, A. (1994). In search of the $21^{\text {st }}$ century nurse for Ghana. International Nursing Review, 41, 118-122.

American Association of Colleges of Nursing. (2008). Essentials of baccalaureate education for professional nursing. Washington, DC: Author. Available at http://www.aacn.nche.edu/Education/pdf/BaccEssentials08.pdf

American International Health Alliance. (2006). Twinning for Health: AIHA Twinning Partnership Toolkit. Retrieved May 4, 2010 from http://www.twinningagainstaids.org/twinningtoolkit/model.html

Anquandah, J. (n.d.). Ghanaian Culture, National Identity and Development. From the National Commission on Culture. Retrieved February 14, 2008 from http://www.ghanaculture.gov.gh/index1.php?linkid=352

Anyangwe, S. C. E., \& Mtonga, C. (2007). Inequities in the global health workforce: The greatest impediment to health in Sub-Saharan Africa. International Journal of Environmental Research and Public Health, 4, 93-100.

Asante, A. D., Zwi, A. B., \& Ho, M. T. (2006). Getting by on credit: How district health managers in Ghana cope with the untimely release of funds. BMC Health Services Research, 6, 105. 
Berry, L. V. (Ed.). (1994). Ghana: A country study. Washington, D.C.: GPO for the Library of Congress. Retrieved March 27, 2010 from the United States Country Studies Index Website@ http://countrystudies.us/ghana/

Billings, D. M., \& Halstead, J. A. (1998). Teaching in nursing: A guide for faculty. Philadelphia, PA: W. B. Saunders.

Boateng, F. (1996-2007). Ghana Life. Retrieved February 14, 2008, from the Yale University Library website http://www.library.yale.edu/ fboateng/fbhp.htm

Bosworth, T. L., Haloburdo, E. P., Hetrick, C., Patchett, K., Thompson, M., \& Welch, M. (2006). International partnerships to promote quality care: Faculty groundwork, student projects, and outcomes. Journal of Continuing Education in Nursing, 37, 32-38.

Buchan, J., \& Calman, L. (2005). Summary: The global shortage of registered nurses: An overview of issues and actions. Geneva, Switzerland: ICN.

Burke, L. A., \& Miller, M. K. (2001). Phone interviewing as a means of data collection: Lessons learned and practical recommendations. Forum: Qualitative Social Research, 2(2), Article 7. Retrieved from http://www.qualitativeresearch.net/index.php/fqs/article/viewArticle/959/2094

Byrne MW. (1998). Productive international faculty exchange: One Columbia University to Gothenburg University example. Journal of Advanced Nursing, 27, 1296-304.

Callister, L. C., \& Cox, A. H. (2006). Opening our hearts and minds: The meaning of international clinical nursing electives in the personal and professional lives of nurses. Nursing and Health Sciences, 8(2), 95-102.

Campinha-Bacote, J. (2012. May). The process of cultural competency in the delivery of healthcare services. Retrieved September 21, 2012 from http://www.transculturalcare.net/

Canadian International Development Agency. (2012, July). Where we work: Sub-Saharan Africa. Retrieved November 21, 2012 from http://www.acdicida.gc.ca/subsaharanafrica

Capacity Plus. (n.d. a.) About us. Retrieved November 21, 2012 from http://www.capacityplus.org/about-us

Capacity Plus. (n.d. b) Ghana. Retrieved November 21, 2012 from http://www.capacityplus.org/ghana

Capacity Plus. (n.d. c.) Knowledge library. Retrieved November 21, 2012 from http://www.capacityplus.org/knowledge-library/all 
Capacity Project. (n.d.). Where we work: Ghana. Retrieved November 27, 2012 from http://www.capacityproject.org/index.php?option=com content\&task=view\&id=1 $\underline{37 \& \text { Itemid }=90}$

Capacity Project. (2009, September). Planning, developing, and supporting the health workforce: Results and lessons learned from The Capacity Project, 2004-2009. Retrieved November 21, 2012 from http://www.capacityproject.org/images/stories/capacity_project_final_report.pdf

Carty, R. M., \& White, J. F. (1996). Strategic planning for international nursing education. Nursing Outlook, 44, 89-93.

Centers for Disease Control and Prevention. (2007, August 17). Progress toward global eradication of dracunculiasis, January 2005--May 2007. Morbidity and Mortality Weekly Report (MMWR), 56, 813-817.

Central Intelligence Agency. (2012, December 5). The world factbook: Ghana. Retrieved December 19, 2012, from https://www.cia.gov/library/publications/the-worldfactbook/geos/gh.html

Chen, H. Y., \& Boore, J. R. P. (2009). Using a synthesized technique for grounded theory in nursing research. Journal of Clinical Nursing, 18, 2251-2260.

Chen, L., Evans, T., Anand, S., Boufford, J. I., Brown, H., Chowdhury, M., ... Wibulpolprasert, S. (2004). Human resources for health: Overcoming the crisis. Lancet, 364, 1984-1990.

Collins, H. (2010). Tacit and explicit knowledge. Chicago, IL: The University of Chicago Press.

Commission on Collegiate Nursing Education. (2009). Standards for accreditation of baccalaureate and graduate degree nursing programs. Washington, DC: Author. Available at http://www.aacn.nche.edu/ccne-accreditation/standards-proceduresresources/baccalaureate-graduate/standards

Cooper, H. (1998). Synthesizing research: A guide for literature reviews $\left(3^{\text {rd }}\right.$ ed.). Thousand Oaks, CA: Sage.

Corbin, J. \& Strauss, A. (2008). Basics of qualitative research: Techniques and procedures for developing grounded theory ( $3^{\text {rd }}$ ed.). Thousand Oaks, CA: Sage.

Crigger, N. J., \& Holcomb, L. (2007). Practical strategies for providing culturally sensitive, ethical care in developing nations. Journal of Transcultural Nursing, $18,70-76$. 
Crossley, M., \& Vulliamy, G. (Eds.). (1997). Qualitative Educational Research in Developing Countries: Current Perspectives. New York Garland Publishing, Inc.

Curtis, V. A., Garbrah-Aidoo, N., \& Scott, B. (2007). Ethics in public health research: Masters in marketing: Bringing private sector skills to public health partnerships. American Journal of Public Health, 97, 634-641.

Cutcliffe, J. R. (2000). Methodological issues in grounded theory. Journal of Advanced Nursing, 31, 1476-1484.

DeSantis, L. (1995). A model for counterparts in interational nursing. International Journal of Nursing Studies, 32, 198-209.

Dickenson-Hazard, N. (2004). Global health issues and challenges. Journal of Nursing Scholarship, 36(1), 6-10.

Diekelmann, N., Schulte, H. D., \& Ironside, P. M. (2002). Developing a science of nursing education: Innovation with research. Journal of Nursing Education, 41, 379-380.

Dodoo, A., Adjei, S., Couper, M., Hugman, B., \& Edwards, R. (2007). When rumours derail a mass deworming exercise. Lancet, 370, 465-466.

Dovlo, D. (2003). The brain drain and retention of health professionals in Africa. [Case study prepared for Regional Training Conference on Improving Tertiary Education in Sub-Saharan Africa: Things That Work, Accra, September 23-25, 2003]. Retrieved from http://www.medact.org/content/health/documents/brain_drain/Dovlo\%20\%20brain\%20drain\%20and\%20retention.pdf

Dovlo, D. (2005). Social dialogue in the health sector: Case study Ghana. [International Labour Office Working Paper 234]. Geneva, Switzerland: International Labour Organization. Retrieved from http://www.ilo.org/wcmsp5/groups/public/--ed_dialogue/---sector/documents/publication/wcms_161165.pdf

Edwards, N., Bunn, H., Mei, W. C., \& Hui, Z. D. (1999). Building community health nursing in the People's Republic of China: A partnership between schools of nursing in Ottawa, Canada, and Tianjin, China. Public Health Nursing, 16, 140145.

Edwards, N., Bunn, H., Morales-Mann, E., Papai, P., \& Davies, B. (2000). International collaborative workshops. A 6-year partnership between Canada and China. Nurse Educator, 25, 88-94. 
Erinosho, O. (Ed.). (2008). Ethics and sustaining research codes for applied social sciences for public health. Proceedings of an International Workshop in collaboration with the Special Programme for Research and Training in Tropical Diseases (TDR) of the World Health Organization, with support of the Federal Ministry of Health, Abuja, Nigeria, April 21-25, 2008. Retrieved December 19, 2012 from http://www.who.int/tdr/publications/non-tdr-publications/ethicspublic-health/en/

Eyiah, J. K. (2004, March 27). Brief history of state-organized education in Ghana. [Feature Article]. Retrieved April 1, 2011 from http://www.ghanaweb.com/GhanaHomePage/features/artikel.php?ID=54812

Ferguson, L., \& Day, R. A. (2005). Evidence-based nursing education: Myth or reality? Journal of Nursing Education, 44, 107-115.

Frenk, J., Chen, L., Bhutta, Z. A., Cohen, J., Crisp, N., Evans, T., ... Zurayk, H. (2010). Health professionals for a new century: Transforming education to strengthen health systems in an interdependent world. The Lancet, 376, 1923-1958.

Geddes, M., Mensah, E. J., Natali, L., \& Quartey, P. (2009, October). OECD DAC-PDE Thematic Study on Untied Aid: Ghana country study. Retrieved November 21, 2012 from the Overseas Development Institute website @ http://www.oecd.org/dac/evaluationofdevelopmentprogrammes/dcdndep/4444773 6.pdf

Ghana Embassy. (2004-2010). About Ghana. Retrieved March 30, 2010, from http://www.ghanaembassy.org/index.php?page=about-ghana

Ghana Health Service. (n.d.). Policy, Planning, Monitoring, and Evaluation Division: Health Research Department. Retrieved December 19, 2012 from http://www.ghanahealthservice.org/division.php?data $=$ unit\&un $=41 \& u m=\% 20 \mathrm{Hea}$ 1th\%20Research\%20Department\&dsion=Policy, \%20Planning\%20,Monitoring $\% 2$ 0and $\% 20$ Evaluation $\% 20$ Division $\& \mathrm{dd}=4$

Ghana Ministry of Health. (2007, September). The Health Sector Programme of Work: 2007-2011: Creating Wealth Through Health. Retrieved December 12, 2012, from http://www.mohghana.org/UploadFiles/Publications/5Yr\%20POW\%20III\%20\%2820072011\%29120422153555.pdf

Ghana Tourism. (2007). Official website of Ghana Tourism. Retrieved February 14, 2008, from http://www.touringghana.com/default.asp\#

Ghana West African Missions. (n.d.). Traveling to Ghana. Retrieved December 19, 2012 from http://www.ghanamission.org/SRH Traveling\%20to\%20Ghana.html 
Girot, E. A., \& Enders, B. C. (2004). International educational partnership for practice: Brazil and the United Kingdom. Journal of Advanced Nursing, 46, 144-151.

Glaser, B., \& Strauss, A. (1967) The discovery of grounded theory. Chicago, IL: Aldine.

Global Health Workforce Alliance. (2008a). Ghana: Implementing a national human resources for health plan. [Task Force on Scaling Up Education and Training for Health Workers: Country Case Study]. Retrieved March 31, 2009 from http://www.who.int/workforcealliance/knowledge/resources/casestudy ghana/en/i ndex.html

Global Health Workforce Alliance. (2008b). Health workers for all and all for health workers: An agenda for global action. Paper presented at the First Global Forum on Human Resources for Health. Retrieved March 31, 2009, from http://www.who.int/workforcealliance/forum/1_agenda4GAction_final.pdf

GNA: Ghana News Agency. (2007, June 8). Guinea worm high in Northern region. Retrieved October 26, 2007 from WordPress weblog@ http://ghana2008.wordpress.com/category/health/

GNA: Ghana News Agency. (2007, October 20). Reviewed curricula for nursing and midwifery training launched. Retrieved October 26, 2007 from http://www.ghananewsagency.org/details/Health/Reviewed-curricula-forNursing-and-Midwifery-Training-launched/?ci=1\&ai=3580

Gordis, D. (2005, November/ December). ACNM partners with community nursing program in Ghana. Quickening, p. 23.

Govier, J. (2004). The Africa chronicle. Canadian Nurse, 100(2), 8-9.

Gyimah, S. O. (2006). Cultural background and infant survival in Ghana. Ethnicity \& Health, 11, 101-120.

Harrison, L., \& Malone, K. (2004). A study abroad experience in Guatemala: Learning first-hand about health, education, and social welfare in a low-resource country. International Journal of Nursing Education Scholarship, 1, Article16.

Haussler, S. C. (2006). Providing a rich experience for nursing faculty visiting from abroad [Chapter 19]. Annual Review of Nursing Education, 363-373.

Hesse, A., Adu-Aryee, N., Entsua-Mensah, K, \& Wu, L. (2006). Knowledge, attitude and practice universal precautions by medical personnel in a teaching hospital. Ghana Medical Journal, 40, 61-64. 
High Level Forum on Aid Effectiveness. (2003). Rome Declaration on Harmonisation. [Product of the $1^{\text {st }}$ High Level Forum on Aid Effectiveness, Rome, Italy, February 24-25, 2003]. Accessed November 16, 2012, from the Organization for Economic Co-operation and Development website @ http://www.oecd.org/dac/aideffectiveness/31451637.pdf

High Level Forum on Aid Effectiveness. (2005). Paris Declaration on Aid Effectiveness. [Product of the $2^{\text {nd }}$ High Level Forum on Aid Effectiveness, Paris, France, March, 2005. Pages 1-14.]. Accessed November 16, 2012, from the Organization for Economic Co-operation and Development website @ http://www.oecd.org/dac/aideffectiveness/34428351.pdf

High Level Forum on Aid Effectiveness. (2008). Accra Agenda for Action. [Product of the $3^{\text {rd }}$ High Level Forum on Aid Effectiveness, Accra, Ghana, September, 2008. Pages 15-21]. Accessed November 16, 2012, from the Organization for Economic Co-operation and Development website @ http://www.oecd.org/dac/aideffectiveness/34428351.pdf

High Level Forum on Aid Effectiveness. (2011a). Busan Partnership for Effective Development Cooperation. [Product of the $4^{\text {th }}$ High Level Forum on Aid Effectiveness, Busan, Korea, November 29 - December 1, 2011]. Retrieved November 21, 2012, from http://www.aideffectiveness.org/busanhlf4/en/about/key-documents.html

High Level Forum on Aid Effectiveness. (2011b). Getting the best from Busan. [Comments from the Honorable Dr. Kwabena Duffuor, Minister for Finance and Economic Planning, Ghana, regarding the $4^{\text {th }}$ High Level Forum and aid effectiveness in his country]. Retrieved November 21, 2012, from http://www.aideffectiveness.org/busanhlf4/newsroom/482-getting-the-best-frombusan.html

High Level Forum on Aid Effectiveness. (n.d.). Ghana: Aid effectiveness in Africal Ghana. [Country report from $4^{\text {th }}$ High Level Forum]. Retrieved November 21, 2012, from http://www.aideffectiveness.org/busanhlf4/en/countries/africa/585.html

Immonen, I., Andersson, N., \& Lvova, M. (2008). Project work across borders in the arctic Barents region: Practical challenges for project members. Nurse Education Today, 28, 841-848.

International Council of Nurses, \& Florence Nightingale International Foundation. (2006). The global nursing shortage: Priority areas for intervention. [Global Nursing Review Initiative Report]. Geneva, Switzerland: Author. 
IntraHealth International. (2007). Learning for performance: A guide and toolkit for health worker training and education programs. Retrieved November 21, 2012, from http://www.intrahealth.org/ intrahea/files/media/learning-forperformance/learning for performance guide.pdf

IRIN. (2008, June 27). Ghana: Conflict in north could threaten elections. Retrieved July 14, 2008, from the UN Office for the Coordination of Humanitarian Affairs @ http://www.IRINnews.org/PrintReport.aspx?ReportId=78993

IRIN. (2008, June 23). Ghana: Vigilante groups fill security vacuum. Retrieved July 14, 2008, from the UN Office for the Coordination of Humanitarian Affairs@, http://www.IRINnews.org/Report.aspx?ReportId=78878

JHPIEGO. (2000-2012). Ghana. Retrieved December 12, 2012, from http://www.jhpiego.org/en/content/ghana

Johnson, P., Ghebreyohanes, G., Cunningham, V., Kutenplon, D., \& Bouey, O. (2007). Distance education to prepare nursing faculty in Eritrea: Diffusion of an innovative model of midwifery education. Journal of Midwifery \& Womens Health, 52, 37- 41.

Keteku, N. W. (1999, November). Educational reform in Ghana: The senior secondary school. Retrieved March 29, 2011, from http://www.bibl.uszeged.hu/oseas_adsec/ghana.htm

Khalil, D. D. (2006). Quality and distribution of continuing professional education opportunities among healthcare workers in Ghana. Journal of Continuing Education in Nursing, 37, 270-279.

Kim, M. J., Woith, W., Otten, K., \& McElmurry, B. J. (2006). Global nurse leaders: lessons from the sages. ANS, Advances in Nursing Science, 29, 27-42.

King, K. (2009). Higher education and international cooperation: The role of academic collaboration in the developing world. In D. Stephens (Ed.), Higher education and international capacity building: Twenty-five years of higher education links (pp. 33-49). Oxford, UK: Symposium Books.

King, N., \& Horrocks, C. (2010). Interviews in qualitative research. Thousand Oaks, CA: Sage.

Kohi, T. W., Portillo, C. J., Safe, J., Okonsky, J., Nilsson, A. C., \& Holzemer, W. L. (2010). The Tanzania HIV/AIDS nursing education (THANE) preservice curriculum. Journal of the Association of Nurses in AIDS Care, 21, 92-98. 
Konadu-Agyemang, K. (2000). The best of times and the worst of times: Structural adjustment programs and uneven development in Africa: The case of Ghana. Professional Geographer, 52, 469-483.

Konadu-Agyemang, K., \& Shabaya, J. (2005). What has corruption got to do with it? Understanding the persistence of rural-urban and inter-regional inequities in Ghana and Zimbabwe. GeoJournal, 62, 129-146.

Kuehn, A. F., Chircop, A., Downe-Wamboldt, B., Sheppard-LeMoine, D., Murnaghan, D., Elliott, J., ... Cardenas, V. (2005). Exploring nursing roles across North American borders. Journal of Continuing Education in Nursing, 36, 153-162.

Kulbok, P. A., Glick, D. F., Mitchell, E. M. \& Greiner, D. (2012) International experiences in nursing education: A review of the literature. International Journal of Nursing Education Scholarship, 9(1), 1-21.

Kulwicki, A. (2006). Improving global health care through diversity. Journal of Transcultural Nursing, 17, 396-397.

Lange, I., \& Ailinger, R. L. (2001). International nursing faculty exchange model: A Chile-USA case. International Nursing Review, 48, 109-116.

Lawson, A. (2010, April 18). The Ghana-Michigan Collaborative Health Alliance for Reshaping Training, Education, and Research (CHARTER). Presented at the African Medical Education Symposium, April 17-19, 2010, in Dar es Salaam, Tanzania. Retrieved December 16, 2012, from The Sub-Saharan African Medical Schools Study web site @ http://www.samss.org/samss.upload/wysiwyg/Presentations\%20from\%20AMES/ Ghana\%20-\%20Michigan.pdf

Leffers, J., \& Mitchell, E. (2011). Conceptual model for partnership and sustainability in global health. Public Health Nursing, 28, 90-102.

Library of Congress, African \& Middle Eastern Divisions, Collections and Services Directorate. (2005, October 4). Selected Internet Resources: Culture: Ghana. Retrieved February 14, 2008 from http://www.loc.gov/rr/international/amed/ghana/resources/ghana-culture.html

Lincoln, Y. S., \& Guba, E. G. (1985). Naturalistic Inquiry. Newbury Park, California: Sage.

Lutzen, K. (2000). A global perspective on domestic and international tensions in knowledge development. Journal of Nursing Scholarship, 32, 335-337.

MacResearchSW. (2007). TAMSAnalyzer. [Software review]. Retrieved December 12, 2012, from http://www.macresearchsw.com/reviews/tamsanalyzer/index.html 
Masera, G., Baez, F., Biondi, A., Cavalli, F., Conter, V., Flores, A., ... Tognoni, G. (1998). North-South twinning in paediatric haemato-oncology: The La Mascota Programme, Nicaragua. Lancet, 352, 1923-1926.

McBrien, B. (2008). Evidence-based care: Enhancing the rigour of a qualitative study. British Journal of Nursing, 17, 1286-1289.

Meerabeau, L. (1992). Tacit nursing knowledge: An untapped resource or a methodological headache? Journal of Advanced Nursing, 17, 108-112.

Melby, C. S., Dodgson, J. E., \& Tarrant, M. (2008). The experiences of western expatriot nursing educators teaching in Eastern Asia. Journal of Nursing Scholarship, 40, 176-183.

Millennium Project. (2006). About the UN Millennium Project. Retrieved May 11, 2011 from http://www.unmillenniumproject.org/who/index.htm

Mock, C., Nguyen, S., Quansah, R., Arreola-Risa, C., Viradia, R., \& Joshipura, M. 2006). Evaluation of trauma care capabilities in four countries using the WHO-IATSIC Guidelines for Essential Trauma Care. World Journal of Surgery, 30, 946-956. doi: 10.1007/s00268-005-0768-4

Mooneyhan, E. L., \& Campos, A. B. (1984). Sharing nursing curriculum with other countries: The need for adaptation. International Nursing Review, 31, 139-144.

Munjanja, O. K., Kibuka, S., \& Dovlo, D. (2005). The nursing workforce in sub-Saharan Africa. [The Global Nursing Review Initiative Issue Paper 7]. Geneva, Switzerland: ICN. Retrieved December 12, 2012, from http://www.hrhresourcecenter.org/node/630

Murphy, C. J. (2008). Focusing on the essentials: Learning for performance. Human Resources for Health, 6(26). doi:10.1186/1478-4491-6-26

Novick, G. (2008). Is there a bias against telephone interviews in qualitative research? Research in Nursing \& Health, 31, 391-398. doi: 10.1002/nur.20259

Ogilvie, L., Allen, M., Laryea, J., \& Opare, M. (2003). Building capacity through a collaborative international nursing project. Journal of Nursing Scholarship, 35, 113-118.

Palmer, D., \& Kramlich, D. (2011). Introduction to the multisystem model of knowledge integration and translation. ANS, Advances in Nursing Science, 34, 29-38. 
Patterson, R., Carter-Templeton, H., \& Russell, C. (2009). Information literacy: Using LISTEN project strategies to equip nurses worldwide. In O. Bodenreider, J. P. Christensen, G. de Moor, A. Famili, U. Fors, A. Hasman et al. (Series Eds.) \& K. Saranto, P. F. Brennan, H. Park, M. Tallberg, \& A. Ensio (Vol. Eds.) Studies in Health Technology and Informatics: Vol. 146. Connecting Health and Humans (pp. 652-656). Amsterdam: IOS Press.

Patterson, R. B., \& Russell, C. K. (2008, February). A research literature synthesis of health disparities and nursing education in Ghana. Poster presented at the 22nd Annual Southern Nursing Research Society Conference, Birmingham, AL.

Patterson, R. B. \& Russell, C. K. (2009, February). A Qualitative Study of Nursing and Nursing Education in Ghana. Poster presented at the 23rd Annual Southern Nursing Research Society Conference, Baltimore, MD. [Poster available for download@ http://www.docstoc.com/docs/49756027/A-Qualitative-Study-ofNursing-and-Nursing-Education-in ].

Pearson Education Inc. (2007). Ghana. [From the Information Please Database.] Retrieved February 14, 2008 from http://www.infoplease.com/ipa/A0107584.html

Pechak, C. M. \& Thompson, M. (2009). A conceptual model of optimal international service-learning and its application to global health initiatives in rehabilitation. Physical Therapy, 89, 1192-1204. doi: 10.2522/ptj.20080378

Perry, S. E., \& Mander, R. (2005). A global frame of reference: Learning from everyone, everywhere. Nursing Education Perspectives, 26, 148-151.

Saldana, J. (2009). The coding manual for qualitative researchers. Thousand Oaks, CA: Sage.

Salvage, J. (2007). Two to tango: The pleasure and pain of international collaboration. [Chapter 3]. In S. M. Weinstein \& A. M. T. Brooks (Eds.). Nursing without borders: Values, wisdom, success markers 9pp. 85-97). Indianapolis, IN: Sigma Theta Tau International.

Schwandt, T. A., \& Halpern, E. S. (1988). Linking auditing and metaevaluation: Enhancing quality in applied research. In L. Bickman (Series Ed.), Applied social research methods series: Vol. 11. Newbury Park, CA: Sage.

Schwimmer, B. (2007). Countries and Their Cultures: Culture of Ghana. Retrieved February 14, 2008 from http://www.everyculture.com/Ge-It/Ghana.html

Shah, M. A., Robinson, T. C., \& Enezi, N. A. (2002). International allied health education and cross-cultural perspectives. Journal of Allied Health, 31, 165-170. 
Smith, E. M. (2005). Telephone interviewing in healthcare research: A summary of the evidence. Nurse Researcher, 12(3), 32-41.

Squires, A. (2008). Language barriers and qualitative nursing research: Methodological considerations. International Nursing Review, 55, 265-273.

Stake, R. E. (2010). Qualitative research: Studying how things work. New York, NY: Guilford Press.

St Jude Children's Research Hospital. (n.d.). International Outreach Program: Guide to Establishing a Pediatric Oncology Twinning Program. Retrieved May 04, 2010, from http://www.stjude.org/SJFile/IOP_Twinning_Manual_082908.pdf

Tabi, M. M., \& Mukherjee, S. (2003). Nursing in a global community: a study abroad program. Journal of Transcultural Nursing, 14, 134-138.

Talley, B. (2006). Nurses and nursing education in Ghana: Creating collaborative opportunities. International Nursing Review, 53, 47-51.

Tavakol, M., Torabi, S., \& Zeinaloo, A. A. (2006). Grounded theory in medical education research. Medical Education Online [serial online], 11:30. Retrieved from http://www.med-ed-online.org

Tennessee Board of Regents. (2009-2010). TBR and University of Cape Coast in Ghana form relationship. Retrieved March 27, 2010, from http://www.tbr.edu/news/default.aspx?id=6016

Tervalon, M., \& Murray-Garcia, J. (1998). Cultural humility versus cultural competence: A critical distinction in defining physician training outcomes in multicultural education. Journal of Health Care for the Poor and Underserved, 9, 117-125.

Tlou, S. D. (1998). International partnerships in nursing education. International Nursing Review, 45, 45-57.

Toumishey, L. H. (1989). Strangers among strangers: Clients and health practitioners in health care settings. Nurse Education Today, 9, 363-367.

University of British Columbia. (2007). E-journals from Ghana. Retrieved October 30, 2007, from https://news.ok.ubc.ca/2007/02/10/e-journals-from-ghana/

U S Department of State. (n.d.). The educational system of Ghana. Retrieved March 29, 2011, from U.S. Embassy Accra, Ghana website @ http://ghana.usembassy.gov/education-of-ghana.html

US Department of State, Bureau of African Affairs. (2010, September 17). Background note: Ghana. Retrieved April 1, 2011, from http://www.state.gov/r/pa/ei/bgn/2860.htm 
University of Tennessee Health Science Center. (2007-2010). The LISTEN Project. Retrieved from https://edtech.uthsc.edu/LISTEN/about.php

Werner, D., Thuman, C., \& Maxwell, J. (2007). Where there is no doctor: A village health care manual. $\left[9^{\text {th }}\right.$ printing]. Berkeley, CA: Hesperian Foundation.

Whitehead, D. (2005). Guest editorial: Empirical or tacit knowledge as a basis for theory development? Journal of Clinical Nursing, 14, 143-144.

World Bank Group. (2010). Ghana: Overview. Retrieved March 30, 2010, from http://go.worldbank.org/LYKT0D6Z50

World Bank Group. (n.d.). Twinning Factsheet. Retrieved December 14, 2012, from http://wbi.worldbank.org/sske/Data/wbi/wbicms/files/drupalacquia/wbi/Twinning_Factsheet.pdf

World Health Organization. (2006). The World Health Report 2006: Working together for health. Geneva, Switzerland: Author. Retrieved from http://www.who.int/whr/2006/whr06 en.pdf

World Health Organization. (2009). Country statistics: Ghana. [Internal query of the Global Health Observatory Data Repository: Nursing and midwifery personnel density]. Retrieved December 14, 2012, from http://apps.who.int/ghodata/?vid=9300\&theme=country

World Health Organization. (2010). Country statistics: Ghana. [Internal query of the Global Health Observatory Data Repository]. Retrieved December 14, 2012, from http://apps.who.int/ghodata/?vid=9300\&theme=country

Wright, M. G., Zerbe, M., \& Korniewicz, D. M. (2001). A critical-holistic analysis of nursing faculty and student interest in international health. Journal of Nursing Education, 40, 229-232.

Zuccala, A. (2006). Modeling the invisible college. Journal of the American Society for Information Science \& Technology, 57, 152-168. 


\section{APPENDIX A. UTHSC IRB APPROVAL LETTER}

\begin{tabular}{lr}
\hline THE UNIVERSITY OF TENNESSEE \\
Health Science Center
\end{tabular}

August 02, 2011

Ramona Bea Patterson, MSN, RN

UTHSC - CON - Nursing- Acute/ Chronic

Re: 11-01439-XM

Study Title: Using Grounded Theory to Explore Nurse Educator Role Transition in an Unfamiliar Setting

\section{Dear Ms. Patterson:}

The Administrative Section of the UTHSC Institutional Review Board (IRB) has received your written acceptance of and/or responses dated 08/01/2011 and 08/02/2011 10:42:17 AM CDT to the provisos outlined in our correspondence of 07/20/2011 and 08/02/2011 concerning the application for the above referenced project. The IRB determined that your application is eligible for exempt review under 45 CFR 46.101(b)(2) in that the study/project involves eligible research using educational tests, surveys, interview procedures, or observation of public behavior. In accord with 45 CFR 46.116(d), informed consent may be altered, with the cover statement used in lieu of an informed consent interview. The requirement to secure a signed consent form is waived under 45 CFR 46.117(c)(2). Willingness of the subject to participate will constitute adequate documentation of consent. Your application has been determined to comply with proper consideration for the rights and welfare of human subjects and the regulatory requirements for the protection of human subjects. Therefore, this letter constitutes full approval of your application, consent cover statement dated 08/02/2011, broad interview guide dated 07/14/2011, follow-up contact script dated 07/14/2011, initial email contact script dated 07/14/2011, and transcriptionist confidentiality statement dated $07 / 11 / 2011$, stamped approved by the $\mathbb{R} B$ on $08 / 02 / 2011$ for the above referenced study.

This study may not be initiated until you receive approval from the institution(s) where the research is being conducted.

In the event that volunteers are to be recruited using solicitation materials, such as brochures, posters, web-based advertisements, etc., these materials must receive prior approval of the IRB.

Any alterations (revisions) in the protocol consent cover statement, broad interview guide, or scripts must be promptly submitted to and approved by the UTHSC Institutional Review Board prior to implementation of these revisions. You have individual responsibility for reporting to the Board in the event of unanticipated or serious adverse events and subject deaths. 
Sincerely,

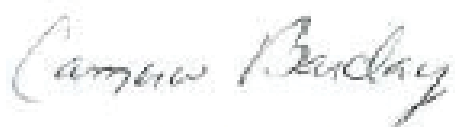

Signature applied by Cameron A Barclay on 08/02/2011 02-50:28 PM CDT

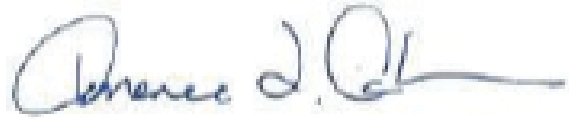

Signature applied by Terrence F Ackerman on 08/02/2011 02:51:13 PM CDT

Cameron Barclay, MSA, CIM, CIP

Director

UTHSC IRB
Terrence F. Ackerman, $\mathrm{Ph}$. D.

Chairman

UTHSC IRB 


\section{APPENDIX B. CONSENT STATEMENT}

Using Grounded Theory to Explore Nurse Educator Role Transition in an Unfamiliar Setting

Principal Investigator: Ramona Patterson, MSN, RN, Nursing $\mathrm{PhD}$ candidate

You are being asked to take part in an interview that is part of a research study to explore insider perspectives and tacit lnowledge (to make them explicit) concerning nurse educator preparation processes for role attaimment in unfamiliar settings. The purpose of this grounded theory research is togenerate a guide for other murse educators preparing for faculty role translation to an unfamiliar setting. This study is the second conducted by a mirse educator doctoral candidate from the United States to increase understanding of mursing education and practice in Ghana, while planning for future capacity building mursing education endeavors there.

Your participation in this research study is voluntary and your refiusal to participate or your decision to withdraw will involve no penalty or loss of benefits to which you are otherwise entitled. Informed consent will be verified by your willinguess to conplete the interview. The interview will be limited to 60 minutes, and your total participation time for this research should be no longer than two hours, including time to review your interview transcript if you choose to do so. Interviews will be audio-recorded and take place by phone or face-to-face. Your interview will start with questions that provide general information about you. Other questions ask you about your experiences and the processes through which you learned how to enact your role in Ghana. The researcher will use an interview guide for broad topics to be covered, including question probes to solicit your insights.

There are no physical risks associated with this study. Some questions may make you feel uncomfortable. Having your voice recorded might make your feel uncomfortable. There is also a potential risk of loss of confidentiality if someone who listens to your audio recording could identify you. Every effort will be made to keep your information confidential; however, this cannot be guaranteed. Your research records will be stored in a locked or password protected location. You will not be identified in any presentations or publications reporting findings from this research study. There are no direct benefits to you for participating in this research. Potential benefits to society relate to helping expand the evidence-base for the professional mursing commmity's tacit knowledge as a foundation to mursing education practices and musing education partnership preparation processes. 


\section{APPENDIX C. LETTER OF INTENT TO NMCG}

\begin{tabular}{|c|c|c|}
\hline \multicolumn{3}{|c|}{$\begin{array}{l}\text { The UNIVERSITY of } \\
\text { TENNESSEE }\end{array}$} \\
\hline & 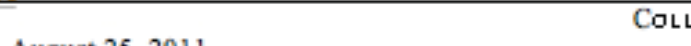 & e HemLth SCIENCEs \\
\hline Date: & August 25, 2011 & 920 Madison Ave., 407 \\
\hline To: & $\begin{array}{l}\text { Ms. Veronica Darko, Registrar/ Chief Executive Officer } \\
\text { Nursing and Midwifery Council of Ghana } \\
\text { PO Box MB44, Accra, Ghana, West Africa }\end{array}$ & $\begin{array}{r}\text { Memphis, TN } 38163 \\
\text { Tel: }(901) 448-5538 \\
(901) 448-8418 \\
\text { Fax: }(901) 448-7291\end{array}$ \\
\hline
\end{tabular}

From: Ramona Patterson, MSN, RX

$\mathrm{PhD}$ candidate (Nursing), College of Graduate Health Sciences

University of Tennessee Health Science Center, Memphis, TN, 38028, USA

Phone: (901) 233-4268.Eax: (901) 867-0487 Email: rpatte16@uthsc.cdu

Honorable Registrar,

I am a nurse educator interested in finding ways to share nurse educator resources around the globe, thereby helping address the human resources for health crisis that exists in many parts of the world. I have a long-time friend in Ghana (Dr Augustine Tawiah), who has sparked my interest in your country, and helped facilitate my initial visit and preliminary research there in 2008 when I conducted an ethnographic study that examined the contexts for nursing education and nursing practice in Ghana. Existing literature to guide individuals through the process of preparing themselves for a nursing education role in an unfamiliar setting is very limited. Much of the existing knowledge resides inside specialized networks or development organizations.

I am currently involved with dissertation research using grounded theory to explore nurse educator's role transition processes as they prepare to work in an unfamiliar setting. I will be interviewing former nursing education partners from the United States (US) and Canada (CAN) who partnered for nursing education in Ghana, and experienced the role transition processes. I would also like to interview key informants in Ghana who have been involved in nursing education partnerships with nurse educators from the US and CAN, to explore their perspectives about the type of information outsiders have needed to enable effective role transition for Ghana. This research is expected to generate a theory that can form the framework to guide individual nurses through the process of acquiring the information and knowledge base necessary for role attainment in an unfamiliar setting. I plan to collect interview data from US and CAN study participants during September and October, and would like to come to Ghana after that to interview key informants there, adding the insider perspective to this grounded theory research.

This letter serves as a letter of my intent, while soliciting your preliminary support of my nursing education research interests, and potential referral to key informants. As the regulatory body for nursing in Ghana, I wanted to make you aware of this research opportunity, gather your insights, and perhaps begin to discuss future opportunities.

Thank you for your time and consideration to this request,

Ramona Patterson, MSN, RN 


\title{
APPENDIX D. LETTER OF INTENT TO GHANA MOH
}

\author{
The LirThas STY of \\ TENNESSEE GI \\ |Date: August 25, 2011 \\ College of Graduate HemLth Seibnces

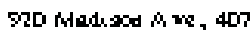 \\ Newpes, TN J J \\ To: Ghana Ministry of Health \\ PO Box M44, Accra, Ghana, West Africa \\ Phone: (+233-302) 684243_Eax: (+233-302) 666366 \\ Tel. (शDI) 442-SSJ \\ (PDI) 442-2413 \\ Far. (POI) 442-729I
}

From: Ramona Patterson, MSN, RX

PhD candidate (Nursing), College of Graduate Health Sciences

University of Tennessee Health Science Center, Memphis, TN, 38028, USA

Phone: (901) 233-4268.Eax: (901) 867-0487 Email: rpatte16@uthsc.edu

Honorable Minister,

I am a nurse educator interested in finding ways to share nurse educator resources around the globe, thereby helping address the human resources for health crisis that exists in many parts of the world. I have a long-time friend in Ghana (Dr Augustine Tawiah), who has sparked my interest in your country, and helped facilitate my initial visit and preliminary research there in 2008 when I conducted an ethnographic study that examined the contexts for nursing education and nursing practice in Ghana. Existing literature to guide individuals through the process of preparing themselves for a nursing education role in an unfamiliar setting is limited. Much of the existing knowledge resides inside specialized networks or development organizations.

I am currently involved with dissertation research using grounded theory to explore nurse educator's role transition processes as they prepare to work in an unfamiliar setting. I will be interviewing former nursing education partners from the United States (US) and Canada (CAN) who partnered for nursing education in Ghana, and experienced the role transition processes. I would also like to interview key informants in Ghana who have been involved in nursing education partnerships with nurse educators from the US and CAN, to explore their perspectives about the type of information outsiders have needed to enable effective role transition for Ghana. This research is expected to generate a theory that can form the framework to guide individual nurses through the process of acquiring the information and knowledge base necessary for role attainment in an unfamiliar setting. I plan to collect interview data from US and CAN study participants during September and early October, and would then like to come to Ghana to interview key informants there, adding the insider perspective to this grounded theory research.

This letter serves as a letter of my intent, while soliciting your preliminary agreement for me to conduct qualitative interviews in Ghana with key informants meeting the study criteria. I understand that the $\mathrm{MoH}$ in Ghana has ethical oversight for health related research there. I am requesting a letter of preliminary approval from your office at this time, to demonstrate to my institution that I have your agreement.

Thank you for your time and consideration to this request,

Ramona Patterson, MSN, RN 


\section{APPENDIX E. LETTER OF INTENT TO GHANA MOE}

The UNIVERSITY of

T ENNESSEE GT

Date: August 25, 2011

College of Gradumte Health Sciences

920 Macison Ave., 407

Memphis, TN 38163

Tel: (901) 448-5538

(901) 448-8418

PO Box M45, Accra, Ghana, West Africa

Phone: (+233-302) 662772 Eax: (+233-302) 664067

Fax: (901) 448-7291

From: Ramona Patterson, MSN, RX

PhD candidate (Nursing), College of Graduate Health Sciences

University of Tennessee Health Science Center, Memphis, TN, 38028, USA

Phone: (901) 233-4268.Eax: (901) 867-0487 Email: rpatte16@uthsc.cdu

Honorable Minister,

I am a nurse educator interested in finding ways to share nurse educator resources around the globe, thereby helping address the human resources for health crisis that exists in many parts of the world. I have a long-time friend in Ghana (Dr Augustine Tawiah), who has sparked my interest in your country, and helped facilitate my initial visit and preliminary research there in 2008 when I conducted an ethnographic study that examined the contexts for nursing education and nursing practice in Ghana. Existing literature to guide individuals through the process of preparing themselves for a nursing education role in an unfamiliar setting is limited. Much of the existing knowledge resides inside specialized networks or development organizations.

I am currently involved with dissertation research using grounded theory to explore nurse educator's role transition processes as they prepare to work in an unfamiliar setting. I will be interviewing former nursing education partners from the United States (US) and Canada (CAN) who partnered for nursing education in Ghana, and experienced the role transition processes. I would also like to interview key informants in Ghana who have been involved in nursing education partnerships with nurse educators from the US and $\mathrm{CAN}$, to explore their perspectives about the type of information outsiders have needed to enable effective role transition for Ghana. This research is expected to generate a theory that can form the framework to guide individual nurses through the process of acquiring the information and knowledge base necessary for role attainment in an unfamiliar setting. I will be collecting interview data from US and CAN study participants during September and October, and then plan to come to Ghana to interview key informants there, adding an insider perspective to this grounded theory research.

This letter serves as a letter of my intent, while soliciting your preliminary support of my educational research interests in Ghana, and potential referral to key informants. I am excited about future opportunities, and very interested in future possibilities to help grow and develop Ghanaian nurse educators.

Thank you for your time and consideration to this request,

Ramona Patterson, MSN, RN 


\section{APPENDIX F. MOH LETTER OF INTRODUCTION AND SUPPORT FOR RESEARCH IN GHANA}

In ease of reply the susuber and the date of ihis fetiet sheuld be quiverst

Out Ret: MON/MRMO/Oooso

Seriat No +

Voup het:

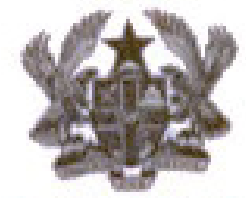

Repulitic of Cibana
MINISTRY OF HEALTH

P.O, BOX M-44

ACCRA

TELEFAX - +233-0302-684250

www, ghanahrbobservalery.ers inforaghanahrhobservatory,erz

$22^{\mathrm{ND}}$ FEBRUARY, 2012

\section{TOWHOM IT MAY CONCERN}

I write to officially introduce to your facility, Ramona Patterson, a nurse educator from the College of Graduate Health Sciences, University of Tennessee Health Science Centre in the United States of America.

Ramona is currently involved with dissertation research using grounded theory to explore nurse educator's role transition processes as they prepare to work in an unfamiliar setting.

In view of that, she is to conduct a qualitative interviews with key informants in Ghana specifically, health facilities/professionals to enrich and facilitate the research work.

Kindly give her the needed support during her visit to your facility.

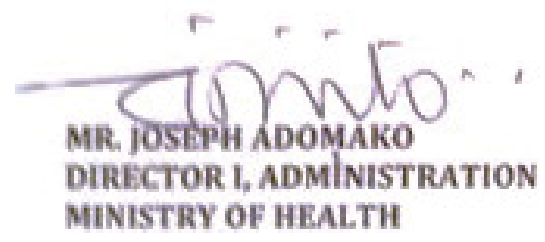




\title{
APPENDIX G. INITIAL EMAIL CONTACT SCRIPT
}

\author{
Research study. Using Grounded Theory to Explore Nurse Educator Role Transition in an \\ Unfamiliar Setting
}

\author{
Study Recruitment: Initial Email Contact Seript
}

You have been identified through literature and Internet searches as a murse educator with experience partnering for nursing education in Ghana. I am a Nursing $\mathrm{PhD}$ candidate at The University of Tennessee Health Science Center in Memphis, Tennessee, establishing a program of research geared toward equipping murse educators for mursing education partnerships in the global community, and networking effectively to address the human resources for health crisis. I began my focused study on international mursing education collaborations in 2007 through independent study with Dr Lynda Wilson, Deputy Director of the PAHO/ WHO Collaborating Center (CC) for International Nursing at the University of Alabama at Birmingham (UAB). Dr Wilson serves as a member of my dissertation committee and has been instrumental in helping focus my dissertation research.

Personal interests related to beginning an endeavor geared toward strengthening mursing capacity in Ghana aligned with a request to UAB for outside help with nursing education at a university in Kumasi Ghana. Personal faith-based contacts in Ghana facilitated my 3-week visit in 2008, when I conducted an ethnographic study to examine the professional, institutional, and Ghanaian contexts that affect nursing education and practice in Ghana. Study findings highlighted the need for more information relative to enacting the nurse educator role effectively in Ghana.

Concurrent with doctoral studies I served as staff development/ murse liaison on a HRSA funded (2007-2010) nurse education, practice, and retention grant geared toward developing information literacy competencies for student and workforce nurses. I also teach Healthcare Informatics to $\mathrm{CNL}$ students. An informatics perspective prompted me to think about the steps for developing information literacy in the field of international nursing education collaborations. In December 2010, I made a podium presentation at the anmual meeting for the Global Alliance of Nursing Education Leadership and Science (GANES) geared toward revealing initial literacy steps that lay the foundation for international nursing education work.

Beyond individual accounts, and internal organizational reports, there is little research evidence to guide individuals new to the filed through the process of faculty role preparation for unfamiliar settings. I am conducting dissertation research titled Using Grounded Theory to Explore Nurse Educator Role Transition in Unfamiliar Settings. I plan to interview approximately 10 murse educators from the United States and Canada who have participated in nursing education endeavors in Ghana, to reveal the profession's tacit knowledge and make it explicit. I also plan to interview key informants in Ghana who have participated in nursing education partnerships with outsiders to reveal the insider perspective concerning role transition information and knowledge processes.

Please consider participating in this research study. If you are interested, you can contact me (Ramona Patterson, MSN, RN) at phone: (901) 233-4268, or email: rpattel6@uthscedu I will answer any questions you may have, provide you with a copy of the consent statement, and plan to follow-up with you within 2 weeks of this initial contact to determine your decision about study participation and to schedule an interview if you are interested in participating.

Thank-you for your time and consideration, 


\title{
APPENDIX H. FOLLOW-UP CONTACT SCRIPT
}

\author{
Research study. Using Grounded Theory to Explore Nurse Educator Role Transition in an \\ Unfamiliar Setting \\ Study Recruitment: Follow-up Contact Script (Phone or Email)
}

I'm following up to see if you are interested in participating in the grounded theory study that explores murse educator's role transition processes when they are enacting their role in an unfamiliar setting. For the puposes of this research the unfamiliar setting is specified as $\mathrm{G}$ hana, Africa. You are being asked to participate in this study because you were identified as an individual with experience working as an outside nurse educator in the Ghana setting.

You are eligible to participate in this study if you meet the following criteria:

- You arels years of age or older

- You are able to speak and converse in English

- You have first hand experience with the role preparation processes for ouside murse educators partnering for mursing education in Ghana.

If you meet these criteria and are interested in being in this study, please read and review the consent statement (sent by email).

After allowing time to review the consent, ask - Do you have any questions related to this study that you would like to ask?

Thank you - those who chose NOT to participate

If subject agrees to participate in the research review the time commitment, schedule or attempt to schedule an interview appointment. Confirm contact \#s by phone, email address, \& home address. Send the consent statement and broad interview guide to all potential participants.

After scheduling the appointment, confirm appointment time - then --..-

Thank you ---

PI: «GreetingLine», MSN, RN 


\title{
APPENDIX I. BROAD INTERVIEW GUIDE
}

\author{
Research study: Using Grounded Theory to Explore Nurse Educator Role Transition in an \\ Unfamiliar Setting
}

\section{BROAD INTERVIEW GUIDE}

Researcher infroduction. Brief overview of study using consent statement to review study purpose, time commitments, confidentiality and recording measures; also verify eligibility.

1. Begin interview (begin recording) and collect demographic data

a. Participant role (host or visiting partner and country)

b. Timefiame for partnership (date of occurrence and duration)

c. Institutional/organizational affiliations in partnership

d. Professional role during partnership (examples: visiting faculty, Fulbright Scholar, distance faculty, host faculty, workshop/ contimuing education instructor, academic administration, regulatory oversight, etc)

e. Prior/ additional experience as outside nurse educator in an unfamiliar setting.

Central question focus: By what processes do outside murse educators leam how to fulfill the nurse educator role in an unfamiliar setting?

f (For North American participants)

Based on your experiences during a nursing education partnership in Ghana, please tell me about how you acquired the knowledge and information you needed as an outsider to address your role as a murse educator in that setting. (Clarify as needed).

g. (For Ghanaian participants)

Based on your experiences with outside murse educators coming into Ghana for nursing education partnerships, please tell me about the processes you have witnessed as outsiders attempted to develop the knowledge base and acquire the information they needed to fulfill their role in Ghana. (Clarify if needed - How did they go about learning what they needed to learn so they could function effectively in Ghana?)

2. Probing questions (examples, not self-limiting)

a. From your experiences, what are the barriers an outside murse educator encounters when developing the knowledge base for assuming the nurse educator role in an unfamiliar setting?

b. Please describe any insider or hidden knowledge that you may have needed, but were challenged to obtain.

c. What are the facilitators an outsider experiences while developing the knowledge base for assuming the murse educator role in an unfamiliar setting?

d. Can you please tell me about any information needs you later realized would have been beneficial to know as advance preparation for the murse educator role?

e. If you can think of anything else you consider important for outsiders to know about preparing for a nurse educator role in an unfamiliar setting, please tell me about it.

Final comments. Solicit referrals to identify additional theoretical sampling candidates. Thank you for participating. Interview ends - recording stops 


\section{APPENDIX J. TRANSCRIPTIONIST CONFIDENTIALITY STATEMENT}

TEUNIVERSITY of

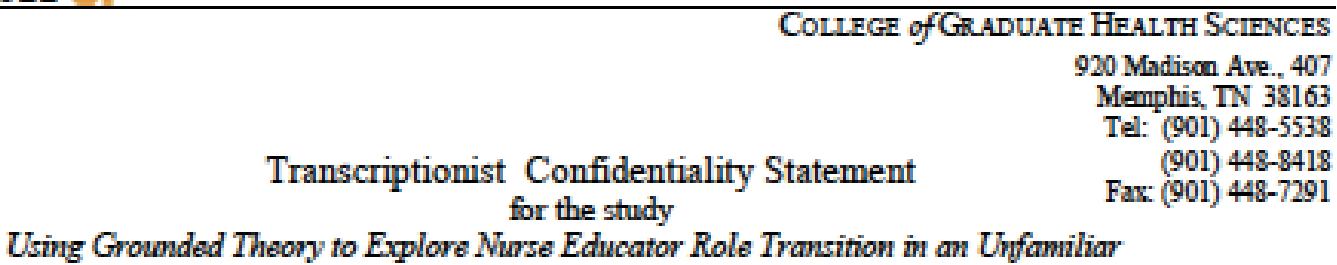

Setting.

You are being asked to read this confidentiality statement, and discuss any questions you may have with the researcher, to confirm your understanding of the ethics concerning confidentiality of study data. Ethics of research require that you NOT talk about any type of study information, including the identity of study participants or disclosure of participant responses, with anyone except the researcher. You understand that there is the possibility that you may identify or think you can identify a participant based on the sound of their voice or their responses to questions but you will maintain your word to keep their identities and responses confidential.

You understand that all research records (audio-tapes, transcribed paper records, and electronic records) will be kept confidential. You understand that when not in use all research records will be stored in a locked or password protected location available only to the researcher.

You understand that at the end of transcriptions ALL copies (paper and electronic) of ALI interviews will be tumed over to the researcher.

You understand that by transcribing interviews you are indicating your agreement with this confidentiality statement for transcribers working on the research study titled Using Grounded Theory to Explore Nurse Educator Role Transition in an Unfamiliar Setting.

\section{Principal Investigator:}

US Phone: (901) 233-4268

US Fax: (901) 867-0487

Preparation date: July 11, 2011 


\section{VITA}

Ramona B. Patterson, born 1956, became a registered nurse in 1979, and received a Master in Nursing Education, with an adult health emphasis, from the University of Memphis in 2006. She has experience in nursing practice, nursing research, and nursing academia. Her areas of clinical expertise include adult health and renal nursing. In addition surgical intensive care and medical-surgical nursing, she spent a number of years working with end-stage renal disease patients as a certified hemodialysis registered nurse, then as a renal research nurse. She is currently serving as an Assistant Professor at Baptist College of Health Sciences, where she teaches nursing research. Her research interests include international nursing education partnerships, information literacy to equip nursing professionals for evidence-based practice regardless of setting, and capacity building efforts in low-resource settings. Ramona successfully defended her dissertation on December 12, 2012, as a requirement for the degree of Doctor of Philosophy with a major in nursing. 